Pacific Journal of Mathematics

FRACTIONAL POWERS OF OPERATORS 


\title{
FRACTIONAL POWERS OF OPERATORS
}

\section{Hikosaburo Komatsu}

\begin{abstract}
A definition of fractional (or complex) powers $A^{\alpha}, \alpha \in C$, is given for closed linear operators $A$ in a Banach space $X$ with the resolvent set containing the negative real ray $(-\infty, 0)$ and such that $\left\{\lambda(\lambda+A)^{-1} ; 0<\lambda<\infty\right\}$ is bounded; fundamental properties such as additivity $\left(A^{\alpha} A^{\beta}=A^{\alpha+\beta}\right)$, coincidence with the iterations $A^{\alpha}=A^{n}$ for integers $\alpha=n$, and analytic dependence on $\alpha$ are discussed. Since the fractional powers $A^{\alpha}$ are generally unbounded in both of the cases $\operatorname{Re} \alpha>0$ and $\operatorname{Re} \alpha<0$, attention is paid to the domains $D\left(A^{\alpha}\right)$, which are related to the spaces $D^{\sigma}$ and $R^{\tau}$ of $x \in X$ defined by the regularity of $(\lambda+A)^{-1} x$ at $\infty$ and 0 . When $-A$ generates a bounded continuous semi-group or a bounded analytic semi-group, more detailed results are obtained.
\end{abstract}

The study of fractional powers of operators has a long history, which may go back to Abel's work on the tautochrone, the RiemannLiouville integral, and its generalizations by M. Riesz. However, it is only recently that the general theory was developed. When $A$ is the negative of the infinitesimal generator of a bounded semi-group of operators, Hille [6] and Phillips [16] showed that fractional powers could be treated in the framework of an operational calculus which they originated. This program was carried out thoroughly by Balakrishnan [1]. Later Balakrishnan [2] gave a new definition and extended his theory to a wider class of operators. About the same time two different definitions were introduced by Krasnosel'skii-Sobolevskii [13] and Kato [10]; further results were obtained by them, Yosida [22], Kato [11] and Watanabe [20]. These theories, with the exception of [11], as well as some classical results on the Riemann-Liouville integral (HardyLittlewood [5], Love-Young [14]), will be reconstructed from a unified point of view.

Our definition of fractional powers is essentially the same as Balakrishnan's second definition and if, in particular, $\operatorname{Re} \alpha>0$, they are identical. In order to see that this definition is a natural one, let us consider the case in which $A$ is bounded and the resolvent set $\rho(A)$ contains the negative real axis $(-\infty, 0]$. The most natural definition of $A^{\alpha}$ for such an operator $A$ is given by the Dunford integral

$$
A^{\alpha}=\frac{1}{2 \pi i} \int_{\Gamma} \zeta^{\alpha}(\zeta-A)^{-1} d \zeta,
$$

where the path $\Gamma$ encircles the spectrum $\sigma(A)$ counterclockwise avoiding 
the negative real axis and $\zeta^{\alpha}$ takes the principal branch. It is then quite easy to see that $A^{\alpha}$ is a bounded operator which is an entire function of $\alpha$, satisfying $A^{\alpha}=A^{n}$ if $\alpha$ is an integer $n$, and $A^{\alpha} A^{\beta}=A^{\alpha+\beta}$, for all $\alpha, \beta \in \mathbf{C}$.

Now we notice that the integration path $\Gamma$ may be considered to encircle the negative real axis clockwise. Thus we can write the integral as

$$
\begin{aligned}
A^{\alpha} & =\frac{1}{2 \pi i} \int_{-\infty}^{0}\left[(\zeta+i 0)^{\alpha}-(\zeta-i 0)^{\alpha}\right](\zeta-A)^{-1} d \zeta \\
& =-\frac{\sin \pi \alpha}{\pi} \int_{0}^{\infty} \lambda^{\alpha}(\lambda+A)^{-1} d \lambda
\end{aligned}
$$

in the sense of Fantappiè and Sato [17]. The last integral loses its ordinary meaning at $\infty$ if $\operatorname{Re} \alpha \geqq 0$ and at 0 if $\operatorname{Re} \alpha \leqq-1$. However, we have a convergent expression valid for $-(m+1)<\operatorname{Re} \alpha<n+1$ :

$$
\begin{aligned}
A^{\alpha}= & -\frac{\sin \pi \alpha}{\pi}\left[\int_{0}^{N}(-1)^{m} \lambda^{\alpha+m}(\lambda+A)^{-1} A^{-m} d \lambda\right. \\
& \left.+\sum_{k=-m}^{n}(-1)^{k+1} \frac{N^{\alpha-k}}{\alpha-k} A^{k}+\int_{N}^{\infty}(-1)^{n+1} \lambda^{\alpha-n-1}(\lambda+A)^{-1} A^{n+1} d \lambda\right],
\end{aligned}
$$

where $N$ is an arbitrary fixed positive real number. This may be proved directly from (1.1). Another proof is obtained from (1.2) by expanding $(\lambda+A)^{-1}$ into a Taylor series around $\infty$ and 0 and applying the formula

$$
\int_{0}^{N} \lambda^{\beta} d \lambda=-\int_{N}^{\infty} \lambda^{\beta} d \lambda=\frac{N^{\beta+1}}{\beta+1},
$$

which holds for every $\beta \neq-1$ in the sense of Fantappiè-Sato.

Throughout this paper we will assume that $A$ is a closed linear operator such that the resolvent set contains $(-\infty, 0)$ and the resolvent satisfies

$$
\left\|\lambda(\lambda+A)^{-1}\right\| \leqq M, \quad \lambda>0,
$$

with a constant $M$ independent of $\lambda$. The bounded operator $A$ considered above and the negatives of infinitesimal generators of bounded semigroups satisfy these conditions. We remark that the operator $A(\lambda+A)^{-1}=1-\lambda(\lambda+A)^{-1}$ is also uniformly bounded. The bound is denoted $L$ :

$$
\left\|A(\lambda+A)^{-1}\right\| \leqq L, \quad \lambda>0 .
$$

The constants $M$ and $L$ will have the same meanings throughout this paper.

Our definition is based on the formula (1.2) or (1.3). But we 
cannot expect that $(\lambda+A)^{-1}$ has a Taylor expansion at $\infty$ or 0 , nor that $A^{\alpha} x$ is defined for all $x$. In place of a Taylor expansion we use the asymptotic expansion of $(\lambda+A)^{-1} x$ which depends on $x$. Thus, our study begins with the regularities of $(\lambda+A)^{-1} x$ at $\infty$ and 0 . If $x$ satisfies

$$
(\lambda+A)^{-1} x=\lambda^{-1} x_{0}-\lambda^{-2} x_{1}+\cdots+(-1)^{n} \lambda^{-n-1} x_{n}+O\left(\lambda^{-\sigma-1}\right)
$$

as $\lambda \rightarrow \infty$, where $n \leqq \sigma<n+1$ (if $n=\sigma, O\left(\lambda^{-\sigma-1}\right)$ must be replaced by $o\left(\lambda^{-\sigma-1}\right)$ ), then $x$ is said to belong to the space $D^{\sigma}$. Similarly the space $R^{\sigma}$ is defined by the regularity at 0 . The spaces $D^{\sigma}$ and $R^{\sigma}$ are shown to be closely related to the domain $D\left(A^{n}\right)$ and the range $R\left(A^{n}\right)$, and give a kind of interpolation spaces (Sections 2,3).

If $\operatorname{Re} \alpha>0$ and if $\sigma>\operatorname{Re} \alpha$, then $A^{\alpha} x$ for $x \in D^{\sigma}$ is defined by (1.2) or its justification as (1.3). It will be proved that the operator $A_{\sigma}^{\alpha}$ thus defined has the smallest closed extension $A_{+}^{\alpha}$ independent of $\sigma$, which we define to be the fractional power. In the same way $A_{-}^{\alpha}$, $\operatorname{Re} \alpha<0$, and $A_{0}^{\alpha}, \alpha \in C$, are defined by their rastrictions to $R^{\sigma}$ and $D^{\sigma} \cap R^{\tau}$, respectively (Section 4 ).

$A_{+}^{\alpha}, A_{-}^{\alpha}$ and $A_{0}^{\alpha}$ are closed linear operators with dense domains in $\overline{D(A)}, \overline{R(A)}$ and $\overline{D(A)} \cap \overline{R(A)}$, respectively, and dense ranges in $\overline{D(A)} \cap \overline{R(A)}$. When $P$ is an operator (or a relation) in a space $X$, and $Y$ is a subspace of $X$, let us call the operator $Q$ in $Y$ defined by $D(Q)=\{y \in D(P) \cap Y ; P y \in Y\}$ and $Q y=P y, y \in D(Q)$, the maximal restriction of $P$ in $Y$. Then $A_{0}^{\alpha}$ for $\operatorname{Re} \alpha>0(\operatorname{Re} \alpha<0)$ is the maximal restriction of $A_{+}^{\alpha}\left(A_{-}^{\alpha}\right)$ in $\overline{D(A)} \cap \overline{R(A)}$. When $\alpha$ is an integer $n, A_{+}^{\alpha}, A_{-}^{\alpha}$ and $A_{0}^{\alpha}$ coincide with the maximal restrictions of $A^{n}$ in $\overline{D(A)}, \overline{R(A)}$ and $\overline{D(A)} \cap \overline{R(A)}$ respectively (Section 4 ).

More precisely the domain $D\left((\mu+A)_{+}^{\alpha}\right)$ does not depend on $\mu \geqq 0$ and coincides with the range $R\left((\mu+A)_{0}^{-\alpha}\right), \mu>0$, while $R\left((\mu+A)_{-}^{-\alpha}\right)$ is contained in $D^{\mathrm{Re} \alpha}$ unless $\operatorname{Re} \alpha$ is an integer and in $D^{\mathrm{Re} \alpha-\varepsilon}$ for any $\varepsilon>0$ if $\operatorname{Re} \alpha$ is an integer. In particular, it follows that $D\left(A_{+}^{\alpha}\right) \subset D\left(A_{+}^{\beta}\right)$ if $\operatorname{Re} \alpha>\operatorname{Re} \beta$. Since $D^{\operatorname{Re} \alpha+\varepsilon} \subset D\left(A_{+}^{\alpha}\right), \varepsilon>0, D^{\operatorname{Re} \alpha}$ is almost equal to $D\left(A_{+}^{\alpha}\right)$. Similarly, if $\mu>0$, we have $D\left(A_{-}^{\alpha}\right)=D\left(\left(A(\mu+A)^{-1}\right)_{-}^{\alpha}\right)=$ $R\left(\left(A(\mu+A)^{-1}\right)_{0}^{-\alpha}\right) \subset R^{-\operatorname{Re} \alpha-\varepsilon}$ or $R^{-\operatorname{Re} \alpha}$ according as $\operatorname{Re} \alpha$ is an integer or not (Section 6).

$A_{ \pm}^{\alpha}$ maps $D^{\sigma} \cap D\left(A_{ \pm}^{\alpha}\right)$ into $D^{\sigma-\operatorname{Re} \alpha}$ if $\alpha-\operatorname{Re} \alpha$ is positive and not an integer.

If $\operatorname{Re} \alpha \cdot \operatorname{Re} \beta>0$, then $A_{*}^{\alpha} A_{*}^{\beta}=A_{*}^{\alpha+\beta}$ holds in the sense of the product of two operators, where the subscripts are assumed to be the same. For every $\alpha$ and $\beta$ the smallest closed extension of $A_{0}^{\alpha} A_{0}^{\beta}$ is the same as $A_{0}^{\alpha+\beta}$. If $x \in D\left(A_{0}^{\beta}\right) \cap D\left(A_{0}^{\alpha+\beta}\right)$, then $A_{0}^{\beta} x \in D\left(A_{0}^{\alpha}\right)$ and $A_{0}^{\alpha} A_{0}^{\beta} x=A_{0}^{\alpha+\beta} x$. Thus the additivity holds fairly completely (Section 7). If $x \in D\left(A_{*}^{\alpha}\right) \cap D\left(A_{*}^{\beta}\right), \operatorname{Re} \alpha>\operatorname{Re} \beta$, then $x$ belongs to $D\left(A_{*}^{\gamma}\right)$ for all 
$\operatorname{Re} \alpha>\operatorname{Re} \gamma>\operatorname{Re} \beta$. Moreover, $A_{*}^{\gamma} x$ is analytic in $\gamma$ and converges strongly to $A_{*}^{\alpha} x\left(A_{*}^{\beta} x\right)$ as $\gamma$ tends to $\alpha(\beta)$ in such a way that $|\arg (\alpha-\gamma)| \leqq \theta<\pi / 2 \quad(|\arg (\gamma-\beta)| \leqq \theta<\pi / 2) . \quad A_{*}^{\gamma} x$ satisfies the convexity inequality

$$
\left\|A_{*}^{\gamma} x\right\| \leqq C\left\|A_{*}^{\alpha} x\right\|^{\theta}\left\|A_{*}^{\beta} x\right\|^{1-\theta}
$$

where $\theta=\operatorname{Re}(\gamma-\beta) / \operatorname{Re}(\alpha-\beta)$ and the constant $C$ depends on $\gamma$ only in terms of $|\arg (\alpha-\gamma)|$ and $|\arg (\gamma-\beta)|$ (Section 8).

Therefore if $B$ is a closed linear operator such that $D(B) \supset D\left(A_{+}^{\gamma}\right)$, we have

$$
\|B x\| \leqq C\left\|A_{+}^{\alpha} x\right\|^{\theta}\|x\|^{1-\theta}, \quad x \in D\left(A_{+}^{\alpha}\right),
$$

where $\operatorname{Re} \alpha>\operatorname{Re} \gamma>0$ and $\theta=\operatorname{Re} \gamma / \operatorname{Re} \alpha$. Conversely if (1.8) holds, then $D(B) \supset D\left(A_{+}^{\gamma}\right)$ for every $\gamma$ with $\operatorname{Re} \gamma>\theta \operatorname{Re} \alpha$ (Section 9).

Now we have to specify the operator $A$ more strictly. An operator $A$ is said to be of type $(\omega, M(\theta)), 0 \leqq \omega<\pi$, if the domain $D(A)$ is dense, the resolvent set of $-A$ contains the sector $|\arg \lambda|<\pi-\omega$ and (1.5) holds on each ray $\lambda=r e^{i \theta}, 0<r<\infty,|\theta|<\pi-\omega$, with $M=M(\theta)$. Every operator $A$ with a dense domain which satisfies (1.5) is of type $(\omega, M(\theta))$ for some $\omega<\pi$. If $-A$ is the generator of a bounded semi-group, then $A$ is of type $(\pi / 2, M \sec \theta)$. Further it is known that $A$ is of type $(\omega, M(\theta))$ with $\omega<\pi / 2$ if and only if $-A$ generates a semi-group $T_{t}$ which has an analytic extension to the sector $|\arg t|<\pi / 2-\omega$ such that the extension is uniformly bounded on each smaller sector $|\arg t| \leqq \pi / 2-\omega-\varepsilon, \varepsilon>0$. Let us call such a semi-group an analytic semi-group.

If $A$ is of type $(\omega, M(\theta))$ and if $0<\alpha<\pi / \omega$, then $A_{+}^{\alpha}$ is of type $\left(\alpha \omega, M_{\alpha}(\theta)\right)$, where $M_{\alpha}(\theta)$ is bounded by $3 M\left((\theta+(\alpha-1) \pi)^{+} / \alpha\right)$. In particular $-A_{+}^{\alpha}$ generates an analytic semi-group $\exp \left(-t A_{+}^{\alpha}\right)$ if $0<\alpha<\pi /(2 \omega)$. The semi-group $\exp \left(-t A_{+}^{\alpha}\right)$ is continuous in the uniform operator topology in $t$ and $\alpha$ in the domain $|\arg t|<\pi / 2-\alpha \omega$, $0<\alpha<\pi /(2 \omega)$. For every $x \in \overline{R(A)}$, $\exp \left(-t A_{+}^{\alpha}\right) x$ converges to $\exp (-t) x$ as $\alpha$ tends to 0 . If $A$ is of type $(\omega, M(\theta))$ and if $0<\alpha<\pi / \omega$ and $\operatorname{Re} \beta>0$, then the multiplicativity $\left(A_{+}^{\alpha}\right)_{+}^{\beta}=A_{+}^{\alpha \beta}$ holds (Section 10).

In case $A$ is the generator of a bounded semi-group $T_{t}$, the value of the fractional power $A_{*}^{\alpha} x$ may be related to the Riemann-Liouville integral of $T_{t} x$. Let $C^{\sigma}, \sigma \geqq 0$, be the set of elements $x$ such that $T_{t} x$ is $n=[\sigma]$ times continuously differentiable and the $n$-th derivative is uniformly Hölder continuous with exponent $\rho=\sigma-n$. Then we have $D^{\sigma} \supset C^{\sigma} \supset D\left(A_{+}^{\alpha}\right)$ for $\operatorname{Re} \alpha=\sigma$. If $\sigma>\operatorname{Re} \alpha>0, A_{+}^{\alpha} x, x \in D^{\sigma}$, is expressed as the fractional derivative of $T_{t} x$ at 0 . If $X$ is sequentially weakly complete, it follows that $x \in D\left(A_{+}^{\alpha}\right)$ if and only if $\left\langle T_{s} x, y^{\prime}\right\rangle \in$ $D\left((-d / d s)_{+}^{\alpha}\right)$ for all $y^{\prime} \in X^{\prime} . \quad$ As in the case of $D^{\sigma}, A_{ \pm}^{\alpha}$ maps $C^{\sigma} \cap D\left(A_{ \pm}^{\alpha}\right)$ 
into $C^{\sigma-\operatorname{Re} \alpha}$ if $\sigma-\operatorname{Re} \alpha$ is positive and not an integer. The semi-group $\exp \left(-t A_{+}^{\alpha}\right)$ generated by $-A_{+}^{\alpha}, 0<\alpha<1$, is uniformly bounded in $t \geqq 0$ and converges strongly to $T_{t}$ as $\alpha$ tends to 1 (Section 11).

Let $-A$ be the generator of a bounded semi-group $T_{t}$. Then $A$ is of type $(\omega, M(\theta))$ with an $\omega<\pi / 2$, or $T_{t}$ is analytic, if and only if there exists a constant $C$ such that

$$
\left\|A_{+}^{\alpha} T_{t}\right\| \leqq C t^{-\operatorname{Re} \alpha} \text {. }
$$

If this is the case, and if $\sigma$ is not an integer, it follows that $C^{\sigma}=D^{\sigma}$, and that $x \in C^{\sigma}$ if only if $\left\|A_{+}^{\alpha} T_{t} x\right\|=O\left(t^{\sigma-\operatorname{Re} \alpha}\right)$ as $t \rightarrow 0$ for $\operatorname{Re} \alpha>\sigma$ (Section 12).

Section 13 deals with the cases where $A$ is a normal operator in a Hilbert space, a restriction, an adjoint operator or a continuous image of another operator which satisfies (1.5). As an application we will prove that the convolution of a $C_{0}^{\alpha}$ function and a $C_{0}^{\beta}$ function belongs to $C_{0}^{\alpha+\beta-\varepsilon}$ for any $\varepsilon>0$, where $C_{0}^{\alpha}$ denotes the class of uniformly Hölder continuous functions with exponent $\alpha$ and with compact supports in $(-\infty, \infty)$. Another application to a nonlinear partial differential equation will also be given. The rest of the section is concerned with the fractional differentiation of functions on the unit circle.

We collect several counterexamples in Section 14, among which there is a simple example of an operator of type $(\pi / 2,2 \sec \theta)$ which does not generate any semi-group of type $(A)$.

The author failed to give an example of operator $A$ such that $-A$ generates a bounded semi-group but for which $D^{\sigma} \neq C^{\sigma}$.

2. Regularity of $(\lambda+A)^{-1} x$ at infinity. If $x \in D\left(A^{n}\right)$ for a nonnegative integer $n,(\lambda+A)^{-1} x$ has the expression

$$
\begin{aligned}
(\lambda+A)^{-1} x= & \lambda^{-1} x-\lambda^{-2} A x+\cdots+(-1)^{n-1} \lambda^{-n} A^{n-1} x \\
& +R_{n-1}(\lambda, x)
\end{aligned}
$$

for $\lambda \in \rho(-A), \lambda \neq 0$, where

$$
\begin{aligned}
R_{k}(\lambda, x) & =(-1)^{k+1} \lambda^{-k-1} A(\lambda+A)^{-1} A^{k} x \\
& =(-1)^{k+1} \lambda^{-k}\left(\lambda^{-1}-(\lambda+A)^{-1}\right) A^{k} x .
\end{aligned}
$$

Because of (1.5) we have the order estimate $R_{n-1}(\lambda, x)=O\left(\lambda^{-n-1}\right)$ as $\lambda$ tends to infinity along the real axis. If $X$ is reflexive, the converse is also true.

THEOREM 2.1. In order that there exist $y_{k} \in X, k=0,1, \cdots, n$, such that

$$
(\lambda+A)^{-1} x=\lambda^{-1} y_{0}-\lambda^{-2} y_{1}+\cdots+(-1)^{n} \lambda^{-n-1} y_{n}+o\left(\lambda^{-n-1}\right)
$$


as $\lambda \rightarrow \infty$, it is necessary and sufficient that $x \in D\left(A^{n}\right), A^{n} x \in \overline{D(A)}$ and $y_{k}=A^{k} x, k=0,1, \cdots, n$. Here the convergence can be either in the weak or strong sense and $\lambda$ may increase, taking only values of a sequence $\lambda_{j} \rightarrow \infty$.

Moreover, let $X$ be reflexive. Then it follows that if $(\lambda+A)^{-1} x$ satisfies

$$
(\lambda+A)^{-1} x=\lambda^{-1} y_{0}-\lambda^{-2} y_{1}+\cdots+(-1)^{n-1} \lambda^{-n} y_{n-1}+O\left(\lambda^{-n-1}\right)
$$

as $\lambda=\lambda_{j} \rightarrow \infty$, there exists a $y_{n} \in X$ such that (2.3) holds. Thus (2.4) is a necessary and sufficient condition in order that $x \in D\left(A^{n}\right)$. In particular we have $\overline{D(A)}=X$, since (2.4) for $n=0$ holds for all $x \in X$.

Proof. In case $n=0$ this is precisely an ergodic theorem due to Hille [6], Kato [9] and Yosida [23]. We shall, however, reproduce the proof for the sake of convenience. If $x \in D(A)$, then we have by (2.1) $(\lambda+A)^{-1} x-\lambda^{-1} x=O\left(\lambda^{-2}\right)=o\left(\lambda^{-1}\right)$. Since $\lambda\left((\lambda+A)^{-1}-\lambda^{-1}\right)$ is uniformly bounded by assumption (1.5), it follows from the BanachSteinhaus theorem that $\lambda\left((\lambda+A)^{-1}-\lambda^{-1}\right) x \rightarrow 0$ strongly for every $x \in \overline{D(A)}$.

Conversely, let the set $\left\{\lambda_{j}\left(\lambda_{j}+A\right)^{-1} x\right\}$ have an accumulation point $y_{0}$ relative to the weak topology, where $\lambda_{j} \rightarrow \infty$. We note that this is the case if $\lambda_{j}\left(\lambda_{j}+A\right)^{-1} x$ converges weakly to $y_{0}$, or if $X$ is reflexive. Clearly $y_{0} \in \overline{D(A)}$, so that we have $\lambda_{j}\left(\lambda_{j}+A\right)^{-1} y_{0} \rightarrow y_{0}$. Thus 0 is a weak accumulation point of $\left\{\lambda_{j}\left(\lambda_{j}+A\right)^{-1}\left(x-y_{0}\right)\right\}$. On the other hand we have for any $\mu \in \rho(-A)$

$$
\begin{aligned}
& (\mu+A)^{-1} \lambda_{j}\left(\lambda_{j}+A\right)^{-1}\left(x-y_{0}\right) \\
& \quad=\lambda_{j}\left(\lambda_{j}+A\right)^{-1}(\mu+A)^{-1}\left(x-y_{0}\right) \rightarrow(\mu+A)^{-1}\left(x-y_{0}\right) .
\end{aligned}
$$

Hence $(\mu+A)^{-1}\left(x-y_{0}\right)$ is the only accumulation point of $\left\{(\mu+A)^{-1} \lambda_{j}\left(\lambda_{j}+A\right)^{-1}\left(x-y_{0}\right)\right\}$. Since $(\mu+A)^{-1}$ is weakly continuous, this implies $(\mu+A)^{-1}\left(x-y_{0}\right)=0$. Thus we have $x=y_{0}$.

Next let $n>0$, and assume that the theorem has been proved for smaller $n$.

If $x \in D\left(A^{n}\right)$ and $A^{n} x \in \overline{D(A)}$, then in (2.1) $\lambda^{n+1} R_{n-1}(\lambda, x)=$ $(-1)^{n} \lambda(\lambda+A)^{-1} A^{n} x$ converges strongly to $(-1)^{n} A^{n} x$ as $\lambda \rightarrow \infty$. This proves (2.3).

Conversely let (2.4) hold. Then by the induction hypotheses we have $x \in D\left(A^{n-1}\right), A^{n-1} x \in \overline{D(A)}$ and $y_{k}=A^{k} ;$ for $k \leqq n-1$. Thus the remainder term in (2.4) is equal to $(-1)^{n^{n} \iota^{-n}}\left(1-\lambda(\lambda+A)^{-1}\right) A^{n-1} x=$ $R_{n-1}(\lambda, x)$. If $X$ is reflexive or if (2.3) holds when $\lambda_{j} \rightarrow \infty,\left\{\lambda_{j}^{n+1} R_{n-1}\left(\lambda_{j}, x\right)\right\}$ has a weak accumulation point $(-1)^{n} y_{n}$. Since $A^{n-1} x \in \overline{D(A)}$ and $(\lambda+A)^{-1}$ 
maps $\overline{D(A)}$ into $\overline{D(A)}, y_{n}$ belongs to $\overline{D(A)}$. We have $(-1)^{n} \lambda_{j}^{n+1} R_{n-1}\left(\lambda_{j}, x\right)=$ $A \lambda_{j}\left(\lambda_{j}+A\right)^{-1} A^{n-1} x$, and $\lambda_{j}\left(\lambda_{j}+A\right)^{-1} A^{n-1} x \rightarrow A^{n-1} x$. Therefore the pair $\left(A^{n-1} x, y_{n}\right)$ belongs to the graph of $A$. This proves $A^{n-1} x \in D(A)$ and $y_{n}=A^{n} x$.

Definition OF THE SPACE $D^{\sigma}$. Let $\sigma \geqq 0$ and $\sigma=n+\rho$ where $n=0,1,2, \cdots$ and $0 \leqq \rho<1$. $\sigma, n$ and $\rho$ will have the same meanings in the following unless the contrary is explicitly stated. We define $D^{\sigma}$ as the set of all $x \in X$ such that $x \in D\left(A^{n}\right)$ and the remainder $R_{n}(\lambda, x)$ in

$$
(\lambda+A)^{-1} x=\lambda^{-1} x-\lambda^{-2} A x+\cdots+(-1)^{n} \lambda^{-n-1} A^{n} x+R_{n}(\lambda, x)
$$

satisfies the order estimate

$$
R_{n}(\lambda, x)=\left\{\begin{array}{lll}
o\left(\lambda^{-\sigma-1}\right) & \text { in case } \rho=0 \\
O\left(\lambda^{-\sigma-1}\right) & \text { in case } \rho>0
\end{array}\right.
$$

Theorem 2.1 shows that $x \in D^{n}$ if and only if $x \in D\left(A^{n}\right)$ and $A^{n} x \in \overline{D(A)}$.

Clearly $D^{\sigma}$ is a vector space. We introduce in it the following topology: By definition a net (directed sequence) $x_{\nu} \in D^{\sigma}$ converges to $x \in D^{\sigma}$ if (i) $A^{k} x_{\nu} \rightarrow A^{k} x$ strongly for $k=0, \cdots, n$, and in case $\rho>0$ if (ii)

$$
\int_{1}^{\infty}<\lambda^{\sigma+1} R_{n}\left(\lambda, x_{\nu}\right), y^{\prime}>d m(\lambda) \rightarrow \int_{1}^{\infty}<\lambda^{\sigma+1} R_{n}(\lambda, x), y^{\prime}>d m(\lambda)
$$

for every $y^{\prime} \in X^{\prime}$ and every measure $m$ on $[1, \infty)$ of finite variation.

When we consider only a (countable) sequence $x_{j}$, the requirement (ii) is simplified as follows.

Proposition 2.2. A sequence $x_{j} \in D^{\sigma}$ converges to $x \in D^{\sigma}$ if and only if (i) $A^{k} x_{j} \rightarrow A^{k} x$ for $k=0, \cdots, n$ and (ii) $\lambda^{\sigma+1} R_{n}\left(\lambda, x_{j}\right)$ is uniformly bounded in $\lambda \geqq 1$ and $j$.

Proof. If (i) holds, then $R_{n}\left(\lambda, x_{j}\right)$ converges strongly to $R_{n}(\lambda, x)$ for every $\lambda$. Thus the sufficiency easily follows from Lebesgue's theorem. To prove the necessity let $x_{j} \rightarrow x_{0}$ in $D^{\sigma}$ and $\left\{\lambda^{\rho+1} R_{n}\left(\lambda, x_{j}\right)\right\}$ be unbounded. Then there is a sequence $\lambda_{k} \geqq 1$ and a $y^{\prime} \in X^{\prime}$ such that $\left.\left\{<\lambda_{k}^{\rho+1} R_{n}\left(\lambda_{k}, x_{j}\right), y^{\prime}\right\rangle\right\}$ is unbounded. On the other hand it follows from the hypothesis that the sequence $\left.\xi_{j}=\left(<\lambda_{k}^{\rho+1} R_{n}\left(\lambda_{k}, x_{j}\right), y^{\prime}\right\rangle\right)$ in the space $m$ of bounded sequences in $k$ converges to $\xi_{0}$ in the weak* topology on $m$ induced by $l^{1}$. Hence $\left\{\xi_{j}\right\}$ is bounded in $m$ contrary to the above. This completes the proof.

Since $A$ is closed we see easily that $D^{n}$ is a Banach space with 
the norm $\|x\|+\|A x\|+\cdots+\left\|A^{n} x\right\|$. When $\rho>0, D^{\sigma}$ is a locally convex space but it is not generally metrizable. There is, however, a semi-norm in $D^{\sigma}$ which will be used as a substitute for a norm.

Set for every $x \in D^{\sigma}$

$$
q_{\sigma}(x)=\sup _{0<\lambda<\infty} \lambda^{\sigma+1}\left\|R_{n}(\lambda, x)\right\| .
$$

This defines a semi-norm in $D^{\sigma}$, because by the definition $\sup _{\lambda \geqq 1} \lambda^{\sigma+1}\left\|R_{n}(\lambda, x)\right\|$ is finite, and if $0<\lambda<1$, we have

$$
\lambda^{\sigma+1}\left\|R_{n}(\lambda, x)\right\|=\lambda^{\sigma-n}\left\|A(\lambda+A)^{-1} A^{n} x\right\| \leqq L\left\|A^{n} x\right\|,
$$

proving that $q_{o}(x)$ is finite for every $x \in D^{\sigma}$. The semi-norm property is clear.

THEOREM 2.3. There exists a constant $C(\sigma)$ depending only on $\sigma, M$ and $L$ such that

$$
\left\|A^{k} x\right\| \leqq C(\sigma) q_{\sigma}(x)^{k / \sigma}\|x\|^{(\sigma-k) / \sigma}
$$

for $x \in D^{\sigma}$ and $k=0,1, \cdots, n$. A set $B$ in $D^{o}$ is bounded if and only if $\sup _{x \in B}\|x\|$ and $\sup _{x} \in_{B} q_{\sigma}(x)$ are finite.

Proof. First we will prove, by induction on $n$, that

$$
\left\|A^{n} x\right\| \leqq\left(2(M L)^{1 / 2}\right)^{n}\left\|A^{n+1} x\right\|^{n /(n+1)}\|x\|^{1 /(n+1)} .
$$

If $x \in D\left(A^{2}\right)$, we have

$$
A x=(\lambda+A)^{-1} A^{2} x+\lambda A(\lambda+A)^{-1} x,
$$

so that

$$
\|A x\| \leqq \lambda^{-1} M\left\|A^{2} x\right\|+\lambda L\|x\|
$$

for any $\lambda>0$. Taking the minimum of the right-hand side, we obtain (2.10) for $n=1$. Let (2.10) have been proved for $n$. Then the proof for $n+1$ is obtained by combining (2.10) for $n$ with

$$
\left\|A^{n+1} x\right\| \leqq 2\left(M L\left\|A^{n+2} x\right\|\left\|A^{n} x\right\|\right)^{1 / 2} .
$$

Similarly from

$$
A^{n} x=A(\lambda+A)^{-1} A^{n} x+\lambda A(\lambda+A)^{-1} A^{n-1} x
$$

and (2.8) we get

$$
\left\|A^{n} x\right\| \leqq(1+\rho) \rho^{-\rho /(1+\rho)} q_{\sigma}(x)^{1 /(1+\rho)}\left(L\left\|A^{n-1} x\right\|\right)^{\rho /(1+\rho)} .
$$

Thus the combination with (2.10) for $n-1$ gives 


$$
\left\|A^{n} x\right\| \leqq C(\rho) q_{o}(x)^{n / \sigma}\|x\|^{\rho / \sigma},
$$

where $C(\rho)=\left[(1+\rho)^{1+\rho} \rho^{-\rho}\left(2(M L)^{1 / 2}\right)^{(n-1) \rho} L^{\rho}\right]^{n / \sigma}$. Now we can prove (2.9) for smaller $k$ step by step using the inequality (2.10).

To prove the second part let $B$ be a bounded set in $D^{\sigma}$. Then in particular sup $\|x\|$ and sup $\left\|A^{n} x\right\|$ are finite. By the same method as in the proof of Proposition 2.2 we can easily show that

$$
\sup _{\lambda \geq 1} \lambda^{\sigma+1}\left\|R_{n}(\lambda, x)\right\|
$$

is finite. Since $\sup _{0<\lambda<1} \lambda^{\sigma+1}\left\|R_{n}(\lambda, x)\right\| \leqq L\left\|A^{n} x\right\|$, we conclude that $\sup q_{\sigma}(x)$ is finite.

Conversely let $\sup _{x \in B}\|x\|$ and $\sup _{x} \in_{B} q_{\sigma}(x)$ be finite. Then by (2.9) $\sup \left\|A^{k} x\right\|$ is finite for $k=0,1, \cdots, n$. Furthermore,

$$
\sup _{x \in B}\left|\int_{1}^{\infty}<\lambda^{\sigma+1} R_{n}(\lambda, x), y^{\prime}>d m\right| \leqq \sup _{x \in B} q_{o}(x)\left\|y^{\prime}\right\||m|
$$

is finite. Thus $B$ is bounded in $D^{\sigma}$. The proof is completed.

The space $D^{\sigma}$ is quasi-complete. Although we do not use this fact, we briefly sketch its proof. Let $x_{\nu}$ be a bounded Cauchy net in $D^{\sigma}$. Then $x_{\nu}$ converges to an $x$ in $D^{n}$ relative to the topology of $D^{n}$. Since $X$ is metrizable and since the mapping which maps $A^{n} x_{\nu} \in X$ into $<\lambda^{\sigma+1} R_{n}\left(\lambda, x_{\nu}\right), y^{\prime}>\in C^{[1, \infty)}$ is continuous, Lebesgue's theorem proves that

$$
\int_{1}^{\infty}<\lambda^{\sigma+1} R_{n}\left(\lambda, x_{\nu}\right), y^{\prime}>\operatorname{dm}(\lambda) \rightarrow \int_{1}^{\infty}<\lambda^{\sigma+1} R_{n}(\lambda, x), y^{\prime}>d m(\lambda) .
$$

We have introduced a sophisticated topology in $D^{\sigma}$. The reason will be explained in the following lemma and theorem.

LEMma 2.4. If $\mu>0$, then $\mu(\mu+A)^{-1}$ is a one-to-one continuous mapping from $D^{\sigma}$ onto $D^{\sigma+1}$. Moreover, for every $x \in D^{\sigma}$

$$
\mu(\mu+A)^{-1} x \rightarrow x\left(D^{o}\right) \text { as } \mu \rightarrow \infty \text {. }
$$

Proof. Let $x^{\mu}=\mu(\mu+A)^{-1} x$ for $x \in D^{o}$. Then we have

$$
(\lambda+A)^{-1} x^{\mu}=\lambda^{-1} y_{0}^{\mu}-\lambda^{-2} y_{1}^{\mu}+\cdots+(-1)^{n} \lambda^{-n-1} y_{n}^{\mu}+R_{n}\left(\lambda, x^{\mu}\right),
$$

where

$$
y_{k}^{\mu}=A^{k} x^{\mu}=\mu(\mu+A)^{-1} A^{k} x
$$

and

$$
\begin{aligned}
R_{n}\left(\lambda, x^{\mu}\right) & =\mu(\mu+A)^{-1} R_{n}(\lambda, x) \\
& =(-1)^{n+1} \lambda^{-n-2} A^{n+1} x^{\mu}-\mu A(\mu+A)^{-1} \lambda^{-1} R_{n}(\lambda, x) .
\end{aligned}
$$


Here we have used the fact that $\mu(\mu+A)^{-1}$ maps $D\left(A^{n}\right)$ into $D\left(A^{n+1}\right)$. The last equality shows that $x^{\mu} \in D^{\sigma+1}$, and the first equality in (2.12) with (1.6) proves that $\lambda^{\sigma+1} R_{n}\left(\lambda, x^{\mu}\right)$ is uniformly bounded. By definition $A^{k} x \in \overline{D(A)}$ for $k=0,1, \cdots, n$, so that $A^{k} x^{\mu}$ converges to $A^{k} x, \mu \rightarrow \infty$. Hence it follows from Proposition 2.2 that $x^{\mu}$ converges to $x$ in the topology of $D^{\sigma}$. We note, however, that $x^{\mu}$ does not necessarily converge to $x$ in the norm $\|x\|+q_{o}(x)$.

The operator $\mu(\mu+A)^{-1}$ is, of course, one-to-one, and, as is clearly seen from (2.12), maps $D^{\sigma}$ continuously into $D^{\sigma+1}$. If $z \in D^{\sigma+1}$, it is easily shown that $\mu^{-1}(\mu+A) x \in D^{\sigma}$. Thus $\mu(\mu+A)^{-1}$ is a mapping onto $D^{\sigma+1}$.

THeOREM 2.5. If $\sigma^{\prime}>\sigma$, then $D^{\sigma^{\prime}} \subset D^{\sigma}, D^{\sigma^{\prime}}$ is dense in $D^{\sigma}$, and the embedding mapping $\varphi_{\sigma}^{\sigma^{\prime}}: D^{\sigma^{\prime}} \rightarrow D^{\sigma}$ is continuous.

Proof. The fact that $D^{\sigma^{\prime}} \subset D^{\sigma}$ and the continuity of $\varphi_{\sigma}^{\sigma^{\prime}}$ are obvious from the definition of $D^{\sigma}$ and its topology. The above lemma proves that $D^{\sigma+1}$ is dense in $D^{\sigma}$, so that $D^{\sigma+m}$ is dense in $D^{\sigma}$ for every nonnegative integer $m$. For a given $\sigma^{\prime}>\sigma$, take an $m$ such that $\sigma+m>\sigma^{\prime}$. Since $D^{\sigma^{\prime}} \supset D^{\sigma+m}, D^{\sigma^{\prime}}$ is dense in $D^{\sigma}$. This completes the proof. $D^{\sigma^{\prime}}$ is not necessarily dense in $D^{\sigma}$ with the norm topology defined by $\|x\|+q_{\sigma}(x)$.

We can introduced a topology in $D\left(A^{n}\right)$ by the norm

$$
\|x\|_{D\left(A^{n}\right)}=\|x\|+\cdots+\left\|A^{n} x\right\| .
$$

(Note that this norm is equivalent to $\|x\|+\left\|A^{n} x\right\|$ because of Theorem 2.3.) From the closedness of $A$ it easily follows that $D\left(A^{n}\right)$ forms a Banach space with this norm. $D\left(A^{n}\right)$ contains $D^{n}$ as a closed subspace. If $X$ is reflexive, they are identical. But this is not necessarily the case otherwise. Moreover, it is easily shown that $D\left(A^{n}\right) \subset D^{\sigma}$, for all $\sigma<n$, that the embedding is continuous, and that it has a dense range. Thus we have the following inclusion relation:

$$
X \supset \overline{D(A)}=D^{\circ} \supset D^{\rho} \supset D(A) \supset D^{1} \supset D^{1+\rho} \supset \cdots .
$$

In case $D(A)$ is not dense in $X$, the spaces $D^{\sigma}$ for $A$ are virtually determined by a restriction of $A$.

THEOREM 2.6. Let $A_{D}$ be the restriction of $A$ to the domain $D\left(A_{D}\right)=\{x \in D(A) ; A x \in \overline{D(A)}\}$. Then $A_{D}$, considered as a closed operator in $\overline{D(A)}$, satisfies (1.5) and has a dense domain. Moreover, the spaces $D^{\sigma}\left(A_{D}\right)$ corresponding to $A_{D}$ are the same as $D^{\sigma}(A)$ corresponding to A. In particular we have $D^{n}(A)=D\left(A_{D}^{n}\right)$ for every integer $n \geqq 0$.

Proof. It is easily shown that $A_{D}$ has a resolvent $\left(\lambda+A_{D}\right)^{-1}$ which 
is equal to the restriction $\left.(\lambda+A)^{-1}\right|_{\overline{D(A)}}$ of $(\lambda+A)^{-1}$ to $\overline{D(A)}$ for $\lambda \in \rho(-A)$. Thus $\left(\lambda+A_{D}\right)^{-1}$ satisfies (1.5). From Theorem 2.1 it follows that for every $x \in \overline{D(A)}, \lambda\left(\lambda+A_{D}\right)^{-1} x \rightarrow x$ as $\lambda \rightarrow \infty$. This shows that $D\left(A_{D}\right)$ is dense in $\overline{D(A)}$. The identity of the spaces $D^{\sigma}(A)$ and $D^{\sigma}\left(A_{\boldsymbol{D}}\right)$ follows easily from Theorem 2.1 .

3. Regularity of $(\lambda+A)^{-1} x$ at zero. A discussion parallel to Section 2 is possible concerning the regularity of $(\lambda+A)^{-1} x$ at zero. Suppose that $x$ belongs to the range $R\left(A^{n}\right)$ and $x=A^{n} y$. Then we have

$$
\begin{aligned}
(\lambda+A)^{-1} x= & A^{n-1} y-\lambda A^{n-2} y+\cdots+(-1)^{n-1} \lambda^{n-1} y \\
& +(-1)^{n} \lambda^{n}(\lambda+A)^{-1} y
\end{aligned}
$$

for any $\lambda \in \rho(-A)$. Hence we have the asymptotic relation

$$
(\lambda+A)^{-1} x=y_{-1}-\lambda y_{-2}+\cdots+(-1)^{n-2} \lambda^{n-2} y_{-n+1}+O\left(\lambda^{n-1}\right)
$$

as $\lambda \rightarrow 0$, where $y_{-k}=A^{n-k} y$. The counterpart of Theorem 2.1 is the following theorem.

THEOREM 3.1. In order that $(\lambda+A)^{-1} x$ satisfy

$$
(\lambda+A)^{-1} x=y_{-1}-\lambda y_{-2}+\cdots+(-1)^{n-1} \lambda^{n-1} y_{-n}+o\left(\lambda^{n-1}\right)
$$

as $\lambda \rightarrow 0$ with $y_{-k} \in X$, it is necessary and sufficient that there exists a $y_{-n} \in D\left(A^{n}\right) \cap \overline{R(A)}$ such that $x=A^{n} y_{-n}$ and $y_{-k}=A^{n-k} y_{-n}$. The remainder term is then given by

$$
R_{-n}(\lambda, x)=(-1)^{n} \lambda^{n}(\lambda+A)^{-1} y_{-n} .
$$

For the necessity it is sufficient that (3.3) holds for a sequence $\lambda_{j} \rightarrow 0$ relative to the weak topology.

Furthermore, let $X$ be reflexive. Then for every $x \in X$ there is an $x_{h} \in N(A)=\{y ; A y=0\}$ such that

$$
(\lambda+A)^{-1} x=\lambda^{-1} x_{h}+o\left(\lambda^{-1}\right), \quad \text { as } \quad \lambda \rightarrow 0,
$$

and $x-x_{h} \in \overline{R(A)}$. The expression $x=x_{h}+\left(x-x_{h}\right)$ with $x_{h} \in N(A)$ and $x-x_{h} \in \overline{R(A)}$ is unique, so that $X$ is decomposed directly:

$$
X=N(A)+\overline{R(A)} \quad(\text { direct }) .
$$

In case $X$ is reflexive and $n>0$ it follows that if (3.2) is satisfied as $\lambda$ tends to 0 taking values of a sequence $\lambda_{j} \rightarrow 0$, then there exists $y_{-n}$ such that (3.3) holds. Thus (3.2) holds if and only if $x \in R\left(A^{n}\right)$.

Proof. We prove the theorem by induction on $n$. Let $x \in R(A)$ 
and $x=A y$. Then

$$
\lambda(\lambda+A)^{-1} x=\lambda A(\lambda+A)^{-1} y \rightarrow 0 \text { as } \lambda \rightarrow 0 .
$$

Thus by the Banach-Steinhaus theorem we have $\lambda(\lambda+A)^{-1} x \rightarrow 0$ for every $x \in \overline{R(A)}$. Conversely let $\lambda_{j}\left(\lambda_{j}+A\right)^{-1} x$ converge weakly to 0 as $\lambda_{j} \rightarrow 0$. Then using $x=\lambda_{j}\left(\lambda_{j}+A\right)^{-1} x+A\left(\lambda_{j}+A\right)^{-1} x$ we see that $x$ is the limit point of $A\left(\lambda_{j}+A\right)^{-1} x \in R(A)$.

In case $X$ is reflexive, choose a sequence $\lambda_{j} \rightarrow 0$ and let $x_{h}$ be a weak accumulation point of $\left\{\lambda_{j}\left(\lambda_{j}+A\right)^{-1} x\right\}$. Since $A \lambda_{j}\left(\lambda_{j}+A\right)^{-1} x \rightarrow 0$ as $\lambda_{j} \rightarrow 0$ and $A$ is closed, it follows that $x_{h} \in D(A)$ and $A x_{h}=0$. 0 is then a weak accumulation point of $\left\{\lambda_{j}\left(\lambda_{j}+A\right)^{-1}\left(x-x_{h}\right)\right\}=$ $\left\{\lambda_{j}\left(\lambda_{j}+A\right)^{-1} x-x_{h}\right\}$. Hence we obtain $x-x_{h} \in \overline{R(A)}$ as above. Clearly the decomposition is unique.

Let $n>0$. If $y_{-n} \in D\left(A^{n}\right) \cap \overline{R(A)}$ and $x=A^{n} y_{-n}$, then (3.3) follows from (3.1) and the theorem for $n=0$. Conversely let (3.2) be true. Then by the induction hypothesis it follows that $y_{-n+1} \in D\left(A^{n-1}\right) \cap \overline{R(A)}$ and $y_{-k}=A^{n-k-1} y_{-n+1}$. The remainder in (3.2) is equal to $R_{-n+1}(\lambda, x)=$ $(-1)^{n-1} \lambda^{n-1}(\lambda+A)^{-1} y_{-n+1}$. Now let $(-1)^{n-1} \lambda_{j}^{-n+1} R_{-n+1}\left(\lambda_{j}, x\right)$ converge weakly to $y_{-n}$ as $\lambda_{j} \rightarrow 0$. Since $A$ is closed and

$$
A\left(\lambda_{j}+A\right)^{-1} y_{-n+1}=y_{-n+1}-\lambda_{j}\left(\lambda_{j}+A\right)^{-1} y_{-n+1} \rightarrow y_{-n+1},
$$

it follows that $y_{-n} \in D(A)$ and $y_{-n+1}=A y_{-n}$. Noticing that $(\lambda+A)^{-1}$ maps $\overline{R(A)}$ into $\overline{R(A)}$ we have also $y_{-n} \in \overline{R(A)}$.

Definition of THE SPACE $R^{\sigma}$. Let $\sigma, n$ and $\rho$ be as in the previous section. The space $R^{\sigma}$ is by definition the set of all $x \in R\left(A^{n}\right) \cap \overline{R(A)}$. such that the remainder $R_{-n}(\lambda, x)$ in (3.3) satisfies

$$
R_{-n}(\lambda, x)=\left\{\begin{array}{lll}
o\left(\lambda^{\sigma-1}\right) & \text { in case } \rho=0 \\
O\left(\lambda^{\sigma-1}\right) & \text { in case } \rho>0
\end{array}\right.
$$

Note that $x \in R^{n}$ if and only if there is a $y_{-n} \in D\left(A^{n}\right) \cap \overline{R(A)}$ such that. $x=A^{n} y_{-n}$.

We introduce a locally convex topology in $R^{\sigma}$ in the same way as. in $D^{\sigma}$ by the following family of semi-norms:

(i) $\left\|y_{-k}\right\|$ for $k=1, \cdots, n$,

(ii) $\left|\int_{0}^{1}<\lambda^{-\sigma+1} R_{-n}(\lambda, x), y^{\prime}>d m(\lambda)\right|$

for all $y^{\prime} \in X^{\prime}$ and all measure $m$ of finite variation on $(0,1]$.

The following theorem which is analogous to Theorem 2.5 permits. us to reduce most of the discussion about $R^{\sigma}$ to the case of $D^{\sigma}$.

THEOREM 3.2. Let $A_{R}$ be the restriction of $A$ to $D\left(A_{R}\right)=D(A) \cap \overline{R(A)}$. 
Then $A_{R}$ satisfies the assumption (1.5) in $R(A)$, is one-to-one and has a dense range there. The spaces $R^{\circ}(A)$ corresponding to $A$ are the same as $R^{\sigma}\left(A_{R}\right)$ corresponding to $A_{R} . \quad y_{-k}$ in (3.3) is precisely $A_{R}^{-k} x$ and the remainder is given by

$$
R_{-n}(\lambda, x)=(-1)^{n} \lambda^{n}\left(\lambda+A_{R}\right)^{-1} A_{R}^{-n} x .
$$

Furthermore, the inverse $A_{R}^{-1}$ has the resolvent

$$
\left(\lambda+A_{R}^{-1}\right)^{-1}=\left.\lambda^{-1} A\left(\lambda^{-1}+A\right)^{-1}\right|_{\overline{R(A)}}
$$

which satisfies (1.5); and $R^{\circ}(A)$ is the same as and is isomorphic to $D^{\sigma}\left(A_{R}^{-1}\right)$.

Proof. First we prove that $A_{R}$ is one-to-one. This is a consequence of Theorem 3.1. In fact, if $x \in D\left(A_{R}\right)$ and $A_{R} x=0$, then

$$
x=\lambda(\lambda+A)^{-1} x+(\lambda+A)^{-1} A_{R} x \rightarrow 0 \text { as } \lambda \rightarrow 0,
$$

so that $x$ must be 0 .

The range $R\left(A_{R}\right)$ is dense in $\overline{R(A)}$ because if $x \in \overline{R(A)}$, then, by Theorem 3.1, $A(\lambda+A)^{-1} x \rightarrow x$ as $\lambda \rightarrow 0$ and $(\lambda+A)^{-1}$ maps $\overline{R(A)}$ into $\overline{R(A)}$. As is easily shown, $\left(\lambda+A_{R}\right)^{-1}$ coincides with the restriction of $(\lambda+A)^{-1}$ to $\overline{R(A)}$ :

$$
\left(\lambda+A_{R}\right)^{-1}=\left.(\lambda+A)^{-1}\right|_{\overline{R(A)}} .
$$

Thus $A_{R}$ satisfies (1.5).

Now it is easy to see that $R^{o}(A)$ coincides with $R^{o}\left(A_{R}\right)$. In particular we have $R^{n}=R\left(A_{R}^{n}\right)$ for every integer $n \geqq 0$.

Since $\lambda+A_{R}^{-1}=\left(\lambda A_{R}+I\right) A_{R}^{-1}$, and since $A_{R}^{-1}$ and $\lambda A_{R}+I, \lambda^{-1} \in \rho(-A)$, are one-to-one, (3.8) follows from (3.9).

It remains to prove that $D^{\sigma}\left(A_{R}^{-1}\right)=R^{\sigma}(A)$. If $\sigma$ is an integer $n$, then we have $D^{n}\left(A_{R}^{-1}\right)=D\left(A_{R}^{-n}\right)=R\left(A_{R}^{n}\right)=R^{n}(A)$. Further, it follows from (3.8) that

$$
\lambda R_{n}\left(A_{R}^{-1}, \lambda, x\right)=-\lambda^{-1} R_{-n}\left(A_{R}, \lambda^{-1}, x\right)
$$

for every $x \in D^{n}\left(A_{R}^{-1}\right)=R^{n}\left(A_{R}\right)$. Now it readily follows that $D^{\sigma}\left(A_{R}^{-1}\right)=$ $R^{o}\left(A_{R}\right)=R^{o}(A)$ and that the topologies of the two spaces are identical. This completes the proof.

The results in $\S 2$ may be translated in terms of $R^{\sigma}$. We just state two of them.

LEMma 3.3. If $\mu>0$, then $A(\mu+A)^{-1}$ is a one-to-one continuous mapping from $R^{\sigma}$ onto $R^{o+1}$. We have also

$$
A(\mu+A)^{-1} x \rightarrow x \quad\left(R^{o}\right) \quad \text { as } \quad \mu \rightarrow 0
$$


for every $x \in R^{\circ}$.

Theorem 3.4. If $\sigma^{\prime}>\sigma$, then $R^{\sigma^{\prime}} \subset R^{\sigma}, R^{\sigma}$ is dense in $R^{\sigma}$ and the embedding map $\psi_{\sigma}^{\sigma^{\prime}}: R^{\sigma^{\prime}} \rightarrow R^{\sigma}$ is continuous.

Before leaving $R^{\sigma}$, let us investigate the relation between $R^{o}$ and $R\left(A^{n}\right)$. As we have shown above, $R^{n}=R\left(A_{R}^{n}\right)$. Therefore, $R^{n} \subset R\left(A^{n}\right)$. If $X$ is reflexive, they coincide with each other because of (3.6). We: define a norm in $R\left(A^{n}\right)$ by

$$
\|x\|_{R\left(\mathbf{\Delta}^{n}\right)}=\inf _{A^{n} y=x}\|y\|_{D\left(\mathbf{\Delta}^{n}\right)} .
$$

Since the set $\left\{y ; A^{n} y=0\right\}$ is closed in $D\left(A^{n}\right), R\left(A^{n}\right)$ becomes a Banach space with this norm. The topology of $R^{n}$ is defined by the samenorm, so that $R^{n}$ is a closed subspace of $R\left(A^{n}\right)$. If $\sigma<n$, we have $R^{o} \supset R\left(A^{n}\right)$. Therefore, there is the following inclusion relation:

$$
\overline{R(A)}=R^{0} \supset R^{\rho} \supset R(A) \supset R^{1} \supset R^{1+\rho} \supset \cdots \text {. }
$$

THE SPACE $D^{\sigma} \cap R^{\tau}$. Let $\sigma$ and $\tau \geqq 0$. We introduce in the set. $D^{\sigma} \cap R^{\tau}$ the weakest topology that makes both the embedding mappings. into $D^{\sigma}$ and into $R^{\tau}$ continuous. Since $D^{\sigma}$ and $R^{\tau}$ are quasi-complete, it follows that $D^{\sigma} \cap R^{\tau}$ is quasi-complete.

Lemma 3.5. Let $\mu>0$. Then $\mu(\mu+A)^{-1}\left(A(\mu+A)^{-1}\right)$ is a oneto-one continuous mapping from $D^{\sigma} \cap R^{\tau}$ onto $D^{\sigma+1} \cap R^{\tau}\left(D^{\sigma} \cap R^{\tau+1}\right.$, respectively). We have also

$$
\begin{array}{rlll}
\mu(\mu+A)^{-1} x \rightarrow x & \left(D^{\sigma} \cap R^{\tau}\right) & \text { as } & \mu \rightarrow \infty \\
\left(A(\mu+A)^{-1} x \rightarrow x\right. & \left(D^{\sigma} \cap R^{\tau}\right) & \text { as } & \mu \rightarrow 0)
\end{array}
$$

for every $x \in D^{\sigma} \cap R^{\tau}$.

Proof. It is enough to prove that $\mu(\mu+A)^{-1} x \in R^{\tau}$, that

$$
\mu(\mu+A)^{-1} x \rightarrow x \quad\left(R^{r}\right) \text { as } \mu \rightarrow \infty,
$$

and the corresponding facts for $A(\mu+A)^{-1}$ and $D^{\sigma}$. If $(\lambda+A)^{-1} x$ satisfies (3.3) with the remainder $R_{-n}(\lambda, x)=(-1)^{n} \lambda^{n}(\lambda+A)^{-1} y_{-n}=O\left(\lambda^{\sigma-1}\right)$, then $x^{\mu}=\mu(\mu+A)^{-1} x$ satisfies

$$
(\lambda+A)^{-1} x^{\mu}=y_{-1}^{\mu}-\lambda \mu_{-2}^{\mu}+\cdots+(-1)^{n-1} \lambda^{n-1} y_{-n}^{\mu}+R_{-n}\left(\lambda, x^{\mu}\right),
$$

where $y_{-k}^{\mu}=\mu(\mu+A)^{-1} y_{-k} \quad$ and $\quad R_{-n}\left(\lambda, x^{\mu}\right)=\mu(\mu+A)^{-1} R_{-n}(\lambda, x)=$ $(-1)^{n} \lambda^{n}(\lambda+A)^{-1} y_{-n}^{\mu}$. Thus $x^{\mu}$ belongs to $R^{\tau}$. Since $y_{-k} \in D\left(A^{k}\right) \subset \overline{D(A)}$, it follows from Lemma 2.4 that $y_{-k}^{\mu} \rightarrow y_{-k}$ as $\mu \rightarrow \infty$, which proves 
$x^{\mu} \rightarrow x\left(R^{\tau}\right)$. The statements about $A(\mu+A)^{-1}$ are proved similarly. Hence it follows that:

THEOREM 3.6. If $\sigma^{\prime} \geqq \sigma$ and $\tau^{\prime} \geqq \tau$, then $D^{\sigma^{\prime}} \cap R^{\tau^{\prime}}$ is densely contained in $D^{\sigma} \cap R^{\tau}$, and the embedding map is continuous.

THEOREM 3.7. Let $A_{B}=A_{D} \cap A_{R}$, or the restriction of $A$ to $D\left(A_{B}\right)=\{x \in D(A) \cap \overline{R(A)}, A x \in \overline{D(A)}\}$. Then $A_{B}$ is one-to-one, the domain $D\left(A_{B}\right)$ and the range $R\left(A_{B}\right)$ are both dense in $\overline{D(A)} \cap \overline{R(A)}$, and $A_{B}$ and $A_{B}^{-1}$ both satisfy assumption (1.5) in $\overline{D(A)} \cap \overline{R(A)}$. Moreover, the spaces $D^{\sigma} \cap R^{\tau}$ corresponding to $A$ remain unchanged if $A$ is replaced by $A_{B}$.

4. Definition of fractional powers of operators. In this section we give the definition of the fractional power $A^{\alpha}$ of an operator $A$ which satisfies the assumption (1.5), separating $\alpha$ into three cases: (i) $\operatorname{Re} \alpha>0$, (ii) $\operatorname{Re} \alpha<0$, and (iii) $\operatorname{Re} \alpha=0$. Let us begin with

THE CASE $\operatorname{Re} \alpha>0$. First, we take a number $\sigma>\operatorname{Re} \alpha$, and define $A_{\sigma}^{\alpha} x, x \in D^{\sigma}$, by

$$
A_{\sigma}^{\alpha} x=A^{\alpha} x \text { if } \alpha \text { is an integer and }
$$

$$
\begin{aligned}
A_{\sigma}^{\alpha} x= & -\frac{\sin \pi \alpha}{\pi}\left[\int_{0}^{N} \lambda^{\alpha}(\lambda+A)^{-1} x d \lambda\right. \\
& \left.+\int_{N}^{\infty} \lambda^{\alpha} R_{n}(\lambda, x) d \lambda+\sum_{k=0}^{n}(-1)^{k+1} \frac{N^{\alpha-k}}{\alpha-k}\right], \quad \text { otherwise. }
\end{aligned}
$$

Here $N$ is an arbitrary fixed positive number and $\lambda^{\alpha}=\exp (\alpha \log \lambda)$. Clearly the integrals converge and the right-hand side represents a continuous linear operator from $D^{\sigma}$ into $X=X_{w}$ equipped with the weak topology. By a simple computation it is seen that $A_{\sigma}^{\alpha} x$ does not depend on $N$ and that if $\sigma<\sigma^{\prime}$, then $A_{\sigma}^{\alpha} x=A_{\sigma}^{\alpha}, x$ for every $x \in D^{\sigma^{\prime}}$.

If $n<\operatorname{Re} \alpha<\sigma$, we may let $N \rightarrow 0$ and obtain

$$
A_{\sigma}^{\alpha} x=(-1)^{n} \frac{\sin \pi \alpha}{\pi} \int_{0}^{\infty} \lambda^{\alpha-n-1} A(\lambda+A)^{-1} A^{n} x d \lambda .
$$

This expression was used by Balakrishnan [2] to define $A^{\alpha}$.

Proposition 4.1. (Balakrishnan [2]). $A_{\sigma}^{\alpha}$ has a closed extension as an operator in $X$, and its smallest closed extension does not depend on $\sigma>\operatorname{Re} \alpha$.

Proof. Since $A$ and $(\lambda+A)^{-1}$ are commutative with $\left(\mu(\mu+A)^{-1}\right)^{l}$, 
$l=1,2, \cdots, \mu>0$, it is easily shown that

$$
A_{\sigma}^{\alpha}\left(\mu(\mu+A)^{-1}\right)^{l} x=\left(\mu(\mu+A)^{-1}\right)^{l} A_{\sigma}^{\alpha} x, x \in D^{\sigma} .
$$

$\left(\mu(\mu+A)^{-1}\right)^{l}$ maps $X$ continuously onto $D\left(A^{\imath}\right)$ and, hence, into $D^{l-\varepsilon}$, $\varepsilon>0$. Thus, the left-hand side of (4.3) represents a continuous linear operator in $X$, if $l>\sigma$.

In order to prove that $A_{\sigma}^{\alpha}$ has a closed extension, let $x_{j} \in D^{\sigma}$ be a sequence such that $x_{j} \rightarrow 0$ and $A_{\sigma}^{\alpha} x_{j} \rightarrow y$ in $X$. Replacing $x$ in (4.3) by $x_{j}$, and taking the limits of both sides, we have

$$
0=\left(\mu(\mu+A)^{-1}\right)^{l} y \text {. }
$$

This proves $y=0$.

Let $\sigma<\sigma^{\prime}$. It follows from Theorem 2.5 and the continuity of $A_{\sigma}^{\alpha}$ that the closure of the graph $G\left(A_{\sigma^{\prime}}^{\alpha}\right)=\left\{\left(x, A_{\sigma^{\prime}}^{\alpha}, x\right) ; x \in D^{\sigma^{\prime}}\right\}$ in $D^{\sigma} \times X_{w}$ is identical with $G\left(A_{\sigma}^{\alpha}\right)$. Since the closure of $G\left(A_{\sigma^{\prime}}^{\alpha}\right)$ in $X_{w} \times X_{w}$ is equal to the closure of its closure in $D^{\sigma} \times X_{w}$, we have the statement. This completes the proof.

The smallest closed extension of $A_{\sigma}^{\alpha}$, whose existence and uniqueness have been proved above, is defined to be the fractional power of $A$ and denoted by $A_{+}^{\alpha}$, or $A^{\alpha}$ if there will be no confusion.

Proposition 4.2. The fractional power $A_{+}^{\alpha}$ coincides with the fractional power $A_{D+}^{\alpha}$ of the operator $A_{D}$. Furthermore, if $\alpha$ is an integer $n$, then $A_{+}^{\alpha}$ is equal to $A_{D}^{n}$, the $n$ times iteration of $A_{D}$. In particular we have $D\left(A_{+}^{n}\right)=D\left(A_{D}^{n}\right)=D^{n}$.

Proof. In view of Theorem 2.6 we see that $A_{\sigma}^{\alpha}$ is the same as $A_{D \sigma}^{\alpha}$. Thus the former part follows.

Now let $x \in D^{n}$. For every $\mu>0$ we have $\mu(\mu+A)^{-1} x \in D^{n+1} \subset D\left(A_{+}^{n}\right)$ and

$$
\begin{aligned}
A_{+}^{n}\left(\mu(\mu+A)^{-1}\right) x & =A^{n}\left(\mu(\mu+A)^{-1}\right) x \\
& =\mu(\mu+A)^{-1} A_{D}^{n} x .
\end{aligned}
$$

Here let $\mu$ tend to infinity. Then we obtain $\mu(\mu+A)^{-1} x \rightarrow x$ and $\mu(\mu+A)^{-1} A_{D}^{n} x \rightarrow A_{D}^{n} x$ by Lemma 2.4. Hence $x \in D\left(A_{+}^{n}\right)$ and $A_{+}^{n} x$ coincides with $A_{D}^{n} x$. Conversely if $x \in D^{\sigma}$ with $\sigma>n$, then by definition we have $A_{+}^{n} x=A^{n} x=A_{D}^{n} x$.

Since $A_{D}$ has a nonvoid resolvent set in $\overline{D(A)}, A_{D}^{n}$ is closed in $\overline{D(A)}$ (cf. Theorem 6.1 of Taylor [18]), and hence in $X$. Thus $A_{D}^{n}$ is an extension of $A_{+}^{n}$.

Proposition 4.3. The domain $D\left(A_{+}^{\alpha}\right)$ is contained and dense in $\overline{D(A)}$, while the range $R\left(A_{+}^{\alpha}\right)$ is contained in $\overline{D(A)} \cap \overline{R(A)}$. 
Proof. Since $D^{\sigma}, \alpha>\operatorname{Re} \alpha$, is contained and dense in $\overline{D(A)}$, the former part is clear. To prove the latter part it suffices to show $R\left(A_{\sigma}^{\alpha}\right) \subset \overline{D(A)} \cap \overline{R(A)}$ for a $\sigma$. As we have seen above $A_{\sigma}^{\alpha}$ is an operator in $\overline{D(A)}$. Thus the range is contained in $\overline{D(A)}$. We remark that $R_{n}(\lambda, x)$ and $A^{k} x$ for $k=1, \cdots, n$ are elements in $R(A)$. Now let $N$ in (4.1) tend to zero. Then the first term and the term $N^{\alpha} x / \alpha$ tend to zero. Therefore, $A_{\sigma}^{\alpha} x$ is a limit point of elements in $\overline{R(A)}$.

Later we will prove that the range $R\left(A_{+}^{\alpha}\right)$ is dense in $\overline{D(A)} \cap \overline{R(A)}$.

Proposition 4.4. $A_{+}^{\alpha}$ commutes with $\left(\mu(\mu+A)^{-1}\right)^{l}$ and $\left(A(\mu+A)^{-1}\right)^{l}$, whenever $\mu>0$ and $l=1,2, \cdots$. Namely, if $x \in D\left(A_{+}^{\alpha}\right)$, then $\left(\mu(\mu+A)^{-1}\right)^{l} x$ and $\left(A(\mu+A)^{-1}\right)^{l} x$ belong to $D\left(A_{+}^{\alpha}\right)$ and

$$
\begin{aligned}
& A_{+}^{\alpha}\left(\mu(\mu+A)^{-1}\right)^{l} x=\left(\mu(\mu+A)^{-1}\right)^{l} A_{+}^{\alpha} x, \\
& A_{+}^{\alpha}\left(A(\mu+A)^{-1}\right)^{l} x=\left(A(\mu+A)^{-1}\right)^{l} A_{+}^{\alpha} x .
\end{aligned}
$$

Proof. (4.4) follows easily from (4.3). (4.5) is proved similarly.

Proposition 4.5. If $x \in D\left(A_{+}^{\alpha}\right)$, then for every $l=1,2, \cdots$

$$
A_{+}^{\alpha}\left(\mu(\mu+A)^{-1}\right)^{l} x \rightarrow A_{+}^{\alpha} x \text { as } \mu \rightarrow \infty .
$$

Conversely if $y_{j}=A_{+}^{\alpha}\left(\mu_{j}\left(\mu_{j}+A\right)^{-1}\right)^{l} x$ exist for an $x \in \overline{D(A)}$, and if $y_{j}$ converges weakly to an element $y \in X$ as $\mu_{j} \rightarrow \infty$, then $x \in D\left(A_{+}^{\alpha}\right)$ and $A_{+}^{\alpha} x=y$.

Especially let $X$ be reflexive. Then $x$ belongs to $D\left(A_{+}^{\alpha}\right)$ if and only if $\left\{A_{+}^{\alpha}\left(\mu_{j}\left(\mu_{j}+A\right)^{-1}\right)^{l} x\right\}$ is bounded for an $l$ and a sequence $\mu_{j} \rightarrow \infty$.

Proof. The first statement is an immediate consequence of Proposition 4.4. The second and third statements follow easily from the closedness of $A_{+}^{\alpha}$.

Lemma A5 in Kato [11] holds without the reflexivity of $X$.

Proposition 4.6. Let $n<\operatorname{Re} \alpha<n+1$ and $x \in D^{n}$. If

$$
(-1)^{n} \frac{\sin \pi \alpha}{\pi} \int_{0}^{N g} \lambda^{\alpha-n-1} A(\lambda+A)^{-1} A^{n} x d \lambda
$$

converges weakly to a $y \in X$ as $N_{j} \rightarrow \infty$, then $x \in D\left(A_{+}^{\alpha}\right)$ and $A_{+}^{\alpha} x=y$. In case $X$ is reflexive, $x \in D\left(A_{+}^{\alpha}\right)$ with $n<\operatorname{Re} \alpha<n+1$ if (4.6) is uniformly bounded for a sequence $N_{j} \rightarrow \infty$.

Proof. Since the integrand belongs to $\overline{D(A)}$, we have $y \in \overline{D(A)}$. If $\mu>0$, then $\mu(\mu+A)^{-1} x \in D^{n+1}$, so that $A_{+}^{\alpha}\left(\mu(\mu+A)^{-1}\right) x$ is defined 
by (4.2). Therefore,

$$
\begin{aligned}
& A_{+}^{\alpha}\left(\mu(\mu+A)^{-1}\right) x \\
& \quad=(-1)^{n} \frac{\sin \pi \alpha}{\pi} \int_{0}^{\infty} \lambda^{\alpha-n-1} A(\lambda+A)^{-1} A^{n} \mu(\mu+A)^{-1} x d \lambda \\
& =\mathrm{w}-\lim _{j \rightarrow \infty}(-1)^{n} \frac{\sin \pi \alpha}{\pi} \int_{0}^{N_{j}} \lambda^{\alpha-n-1} A(\lambda+A)^{-1} A^{n} \mu(\mu+A)^{-1} x d \lambda \\
& =\mu(\mu+A)^{-1} \mathrm{w}-\lim _{j \rightarrow \infty}(-1)^{n} \frac{\sin \pi \alpha}{\pi} \int_{0}^{N_{j}} \lambda^{\alpha-n-1} A(\lambda+A)^{-1} A^{n} x d \lambda \\
& =\mu(\mu+A)^{-1} y .
\end{aligned}
$$

This shows, by Proposition 4.5, that $x \in D\left(A_{+}^{\alpha}\right)$ and $y=A_{+}^{\alpha} x$.

Proposition 4.7. If $x \in D^{\sigma}$, then $A_{+}^{\alpha} x$ is an analytic function of $\alpha$ on the strip $0<\operatorname{Re} \alpha<\sigma$. Moreover,

$$
\left\|A_{+}^{\alpha} x\right\| \leqq C(\sigma, \alpha) q_{\sigma}(x)^{\operatorname{Re} \alpha / \sigma}\|x\|^{(\sigma-\operatorname{Re} \alpha) / \sigma}
$$

holds for every $x \in D^{\sigma}$ with a constant $C(\sigma, \alpha)$ depending only on $\sigma$ and $\alpha$.

Proof. It is clear by (4.1) that $A_{+}^{\alpha} x$ is analytic in $\alpha$ unless $\alpha$ is an integer $n$. But $\alpha=n$ is not a singularity because $A_{+}^{\alpha} x \rightarrow A^{n} x$ as $\alpha$ tends to $n$. Estimating each term in (4.1) we obtain

$$
\begin{aligned}
\left\|A_{+}^{\alpha} x\right\| & \leqq \\
& +\frac{\sin \pi \alpha}{\pi} \mid\left[\frac{N^{\operatorname{Re} \alpha}}{\operatorname{Re} \alpha} M\|x\|+\sum_{k=0}^{n} \frac{N^{\operatorname{Re} \alpha-k}}{|\alpha-k|}\left\|A^{k} x\right\|\right. \\
& \left.=q_{\sigma}(x)\right] .
\end{aligned}
$$

$|\sin \pi \alpha| /|\alpha-k|$ is bounded by const $e^{\pi|\operatorname{Im} \alpha|}$, and we have by Theorem 2.3 the estimates

$$
\left\|A^{k} x\right\| \leqq C(\sigma)\left(N^{k-\sigma} q_{\sigma}(x)+N^{k}\|x\|\right), k=0, \cdots, n, 0<N .
$$

Hence it follows that

(4.7) $\left\|A_{+}^{\alpha} x\right\| \leqq C_{1}(\sigma) e^{\pi|\operatorname{Im} \alpha|}\left[\frac{|\alpha|}{\operatorname{Re} \alpha} N^{\operatorname{Re} \alpha}\|x\|+\frac{|\alpha-n|}{\sigma-\operatorname{Re} \alpha} N^{\operatorname{Re} \alpha-\sigma} q_{\sigma}(x)\right]$,

with a constant $C_{1}(\sigma)$ depending only on $\sigma$. Taking the minimum of the right-hand side we obtain the desired inequality.

Proposition 4.8. Let $\operatorname{Re} \alpha>0$ and $m=1,2,3, \cdots$. If $\sigma>m+$ $\operatorname{Re} \alpha$, then

$$
A_{D}^{m} A_{+}^{\alpha} x=A_{+}^{\alpha} A_{D}^{m} x=A_{+}^{m+\alpha} x, \quad x \in D^{\sigma} .
$$


Proof. By definition $x \in D^{\sigma}$ if and only if $x \in D\left(A_{D}^{m}\right)$ and $A_{D}^{m} x \in D^{\sigma-m}$. Thus $A_{D}^{m}$ belongs to $D\left(A_{+}^{\alpha}\right)$. Making use of expansion (3.1), we have

$$
\begin{aligned}
\int_{0}^{N} \lambda^{\alpha}(\lambda+A)^{-1} A_{D}^{m} x d \lambda= & (-1)^{m} \int_{0}^{N} \lambda^{m+\alpha}(\lambda+A)^{-1} x d \lambda \\
& +\sum_{k=0}^{m-1}(-1)^{m+k+1} \frac{N^{\alpha+m-k}}{\alpha+m-k} A^{k} x .
\end{aligned}
$$

In view of definition (4.1), this proves that

$$
A_{\sigma-m}^{\alpha} A_{D}^{m} x=A_{\sigma}^{m+\alpha} x, \quad x \in D^{\sigma} .
$$

Notice that the integrals defining $A^{\alpha} x$ converge in the sense of Riemann and that the integrands are commutative with $A_{D}^{m}$. Then the former part of (4.8) follows easily from the closedness of $A_{D}^{m}$.

Proposition 4.9. Let $\operatorname{Re} \alpha, \operatorname{Re} \beta>0$, and $\sigma$ be sufficiently large. Then we have

$$
A_{+}^{\alpha} A_{+}^{\beta} x=A_{+}^{\alpha+\beta} x, \quad x \in D^{\sigma} .
$$

Proof. Combining Proposition 4.7 with Proposition 4.8 we see that if $m$ is an integer such that $0 \leqq m<\sigma$, then for every $x \in D^{\sigma}, A_{+}^{\beta} x$ is a $D^{m}$ valued analytic function of $\alpha$ on the strip $0<\operatorname{Re} \beta<\sigma-m$. If $\operatorname{Re} \alpha<m, A_{+}^{\alpha}$ is a continuous operator from $D^{m}$ into $X$. Thus $A_{+}^{\alpha} A_{+}^{\beta} x$ is analytic in $\beta$ in the domain defined by $0<\operatorname{Re} \alpha<m$ and $0<\operatorname{Re} \beta<\sigma-m$.

From Proposition 4.7 it follows that $A_{+}^{\alpha} A_{+}^{\beta} x$ is analytic in $\alpha$ in the same domain. Therefore, it is analytic in $\alpha$ and $\beta$. Similarly $A^{\alpha+\beta} x$ is analytic in $\alpha$ and $\beta$.

Thus in order to prove (4.9) in general it is enough to prove it for $0<\operatorname{Re} \alpha+\operatorname{Re} \beta<1$ and say for all $x \in D\left(A^{2}\right)$. We have by (4.2)

$$
\begin{aligned}
A_{+}^{\alpha} A_{+}^{\beta} x & =\frac{\sin \pi \alpha}{\pi} \frac{\sin \pi \beta}{\pi} \int_{0}^{\infty} \int_{0}^{\infty} \lambda^{\alpha-1} \tau^{\beta-1}(\lambda+A)^{-1}(\tau+A)^{-1} A^{2} x d \lambda d \tau \\
& =\frac{\sin \pi \alpha}{\pi} \frac{\sin \pi \beta}{\pi} \int_{0}^{\infty} \int_{0}^{\infty} \lambda^{\alpha-1} \tau^{\beta-1} \frac{\lambda(\lambda+A)^{-1}-\tau(\tau+A)^{-1}}{\lambda-\tau} A x d \lambda d \tau .
\end{aligned}
$$

Since the integrand is analytic, we may take for the path of integration of $\lambda$ a line parallel to and slightly above the real axis and for $\tau$ a line slightly under the real axis. Splitting the integrand into two terms, we obtain

$$
A_{+}^{\alpha} A_{+}^{\beta} x=-\frac{\sin \pi \alpha}{\pi} \frac{\sin \pi \beta}{\pi}\left[\int_{0}^{\infty} \lambda^{\alpha}(\lambda+A)^{-1} A x d \lambda \int_{0}^{\infty} \tau^{\beta-1}(\tau-\lambda)^{-1} d \tau\right.
$$




$$
\begin{aligned}
& \left.+\int_{0}^{\infty} \tau^{\beta}(\tau+A)^{-1} A x d \tau \int_{0}^{\infty} \lambda^{\alpha-1}(\lambda-\tau)^{-1} d \lambda\right] \\
= & \frac{\sin \pi \alpha}{\pi} \int_{0}^{\infty} \lambda^{\alpha}(\lambda+A)^{-1} A x(-\lambda-i 0)^{\beta-1} d \lambda \\
& +\frac{\sin \pi \beta}{\pi} \int_{0}^{\infty} \tau^{\beta}(\tau+A)^{-1} A x(-\tau+i 0)^{\alpha-1} d \tau \\
= & \frac{\sin \pi(\alpha+\beta)}{\pi} \int_{0}^{\infty} \lambda^{\alpha+\beta-1}(\lambda+A)^{-1} A x d \lambda=A_{+}^{\alpha+\beta} x .
\end{aligned}
$$

The CASE $\operatorname{Re} \alpha<0$. Similarly as in the case $\operatorname{Re} \alpha>0$, we define $A_{-\sigma}^{\alpha} x$ for $x \in R^{\sigma}$ and for $-\sigma<\operatorname{Re} \alpha<0$ by

$$
\begin{aligned}
A_{-\sigma}^{\alpha} x= & y_{\alpha} \text { if } \alpha \text { is an integer or } \\
= & -\frac{\sin \pi \alpha}{\pi}\left[\int_{0}^{N} \lambda^{\alpha} R_{-n}(\lambda, x) d \lambda\right. \\
& \left.+\sum_{k=1}^{n}(-1)^{k+1} \frac{N^{\alpha+k}}{\alpha+k} y_{-k}+\int_{N}^{\infty} \lambda^{\alpha}(\lambda+A)^{-1} x d \lambda\right] \text { otherwise, }
\end{aligned}
$$

where $R_{-n}(\lambda, x)$ and $y_{-k}$ are as in (3.3) and (3.4) and $N$ is an arbitrary fixed positive number. $A_{-\sigma}^{\alpha} x$ does not depend on $N$ and $\sigma$ as far as it has a meaning.

Now we might pursue the analogy of the case $\operatorname{Re} \alpha>0$ and could prove that $A_{-\sigma}^{\alpha}$ has the smallest closed extension independent of $\sigma$, etc. We remark, however, that $A_{-\sigma}^{\alpha}$ defined above is the same as $\left(A_{R}^{-1}\right)_{\sigma^{-\alpha}}$. For, the domains $R^{\sigma}(A)$ and $D^{\sigma}\left(A_{R}^{-1}\right)$ are one and the same as was shown in Theorem 3.2, and it is easily proved by (3.10) that the expression (4.10) is identical with the expression (4.1) for $A_{R}^{-1}$.

Consequently we have the following proposition as a corollary of Propositions 4.1-4.9.

Proposition 4.10. Let $\operatorname{Re} \alpha<0$ and $A_{-\sigma}^{\alpha}$ be defined by (4.10). Then $A_{-\sigma}^{\alpha}$ has the smallest closed extension $A_{-}^{\alpha}$ in $X$ which is independent of $\sigma . \quad A_{-}^{\alpha}$ is identical with $A_{R-}^{\alpha}=\left(A_{R}^{-1}\right)_{+}^{-\alpha}$ defined by $A_{R}$ and if especially $\alpha$ is a negative integer $-n, A_{-}^{\alpha}$ coincides with $A_{R}^{-n}$ in the usual sense. The domain $D\left(A^{\alpha}\right)$ is contained and dense in $\overline{R(A)}$, and the range $R\left(A_{-}^{\alpha}\right)$ is contained $\overline{D(A)} \cap \overline{R(A)}$. $\quad A^{\alpha}$ is commutative with bounded operators $\left(\mu(\mu+A)^{-1}\right)^{l}$ and $\left(A(\mu+A)^{-1}\right)^{l}, \mu>0, l=1,2, \cdots$.

For every $x \in R^{o}, A^{\alpha} x$ is analytic in $\alpha$ on the strip $-\sigma<\operatorname{Re} \alpha<0$. If $\operatorname{Re} \alpha, \operatorname{Re} \beta<0$, then there is a $\sigma \geqq 0$ such that

$$
A^{\alpha} A_{-}^{\beta} x=A^{\alpha+\beta} x
$$

holds for every $x \in R^{o}$. 
THE CASE $\operatorname{Re} \alpha=0$. If $\operatorname{Re} \alpha=0$, the integral (1.2) becomes singular both at infinity and at zero. Therefore, first we restrict the domain to $D^{\sigma} \cap R^{\tau}$, where $\sigma=n+\rho$ and $\tau=m+\pi$ are positive. $A^{\alpha} x$ for $x \in D^{\sigma} \cap R^{\tau}$ is defined by

$$
\begin{aligned}
A_{\sigma \tau}^{\alpha} x= & x \text { if } \alpha=0 \text { or } \\
= & -\frac{\sin \pi \alpha}{\pi}\left[\int_{0}^{N} \lambda^{\alpha} R_{-m}(\lambda, x) d \lambda\right. \\
& \left.+\sum_{k=-m}^{n}(-1)^{k+1} \frac{N^{\alpha-k}}{\alpha-k} A_{B}^{k} x+\int_{N}^{\infty} \lambda^{\alpha} R_{n}(\lambda, x) d \lambda\right], \text { otherwise. }
\end{aligned}
$$

As before $N$ is an arbitrary fixed positive number, and it has nothing to do with the value of $A_{\sigma \tau}^{\alpha} x$.

The right-hand side of (4.12) expresses an analytic function of $\alpha$ on the strip $-\tau<\operatorname{Re} \alpha<\sigma$ and it coincides with $A_{-\tau}^{\alpha} x$ and $A_{\sigma}^{\alpha} x$ in the subdomain $-\tau<\operatorname{Re} \alpha<0$ and $0<\operatorname{Re} \alpha<\sigma$, respectively, Thus it is possible to give another definition of fractional powers by means of the operator $A_{\sigma \tau}^{\alpha}$ even in the case $\operatorname{Re} \alpha \neq 0$.

Proposition 4.11. For every complex number $\alpha, A_{\sigma \tau}^{\alpha}$ has the smallest closed extension $A_{0}^{\alpha}$ independent of $\sigma$ and $\tau$ such that $-\tau<\operatorname{Re} \alpha<\sigma$.

Proof. This may be proved in the same way as Proposition 4.1 by making use of operators $\left(\mu(\mu+A)^{-1}\right)^{l}\left(A(\nu+A)^{-1}\right)^{h}$ in place of $\left(\mu(\mu+A)^{-1}\right)^{l}$.

Proposition 4.12. $A_{0}^{\alpha}$ coincides with the fractional power $\left(A_{B}\right)_{0}^{\alpha}$ of the operator $A_{B}$. If $\alpha$ is an integer $n, A_{0}^{\alpha}$ is equal to $A_{B}^{n}$.

In case $\operatorname{Re} \alpha>0(\operatorname{Re} \alpha<0), A_{0}^{\alpha}$ is the restriction of $A_{+}^{\alpha}\left(A_{-}^{\alpha}\right)$ to the domain $D\left(A_{0}^{\alpha}\right)=D\left(A_{+}^{\alpha}\right) \cap \overline{R(A)}\left(D\left(A_{-}^{\alpha}\right) \cap \overline{D(A)}\right.$, respectively).

Proof. In view of Theorem 3.7, the first statement is clear. The second statement follows from the third statement and Proposition 4.2 and 4.10 .

To prove the third statement, let $\operatorname{Re} \alpha>0$. Obviously $A_{0}^{\alpha}$ is a restriction of $A_{+}^{\alpha}$ and we have $D\left(A_{0}^{\alpha}\right) \subset D\left(A_{+}^{\alpha}\right) \cap \overline{R(A)}$. Conversely let $x \in D\left(A_{+}^{\alpha}\right) \cap \overline{R(A)}$. If $l$ is sufficiently large, $x^{\mu \nu}=\left(\mu(\mu+A)^{-1}\right)^{l} A(\nu+A)^{-1} x$ belongs to $D^{l-\varepsilon} \cap R^{1-z} \subset D\left(A_{0}^{\alpha}\right)$, and it follows from Proposition 4.4 and Theorem 3.5 that $x^{\mu \nu} \rightarrow x$ and $A_{0}^{\alpha} x^{\mu \nu} \rightarrow A_{+}^{\alpha} x$ as $\mu \rightarrow \infty$ and $\nu \rightarrow 0$. Thus we have $x \in D\left(A_{0}^{\alpha}\right)$. The proof in the case $\operatorname{Re} \alpha<0$ is similar.

Analogously to Proposition 4.9 we have 
Proposition 4.13. Let $\alpha$ and $\beta$ be complex numbers. If $\sigma$ and $\tau$ are sufficiently large, we have

$$
A_{0}^{\alpha} A_{0}^{\beta} x=A_{0}^{\alpha+\beta} x, \quad x \in D^{\sigma} \cap R^{\tau} .
$$

Proof. We omit the details of the proof which is essentially the same as that of Proposition 4.9. We note only that if an integer $m$ satisfies $-\tau<m<\sigma$, then $A_{B}^{m}$ maps $D^{\sigma} \cap R^{\tau}$ onto $D^{\sigma-m} \cap R^{\tau+m}$ homeomorphically and that if, in addition, the real parts of a complex number $\alpha$ and $\alpha+m$ satisfy the same condition, then we have

$$
A_{0}^{\alpha} A_{B}^{m} x=A_{B}^{m} A_{0}^{\alpha} x=A_{0}^{\alpha+m} x, \quad x \in D^{\sigma} \cap R^{\tau} .
$$

5. The case where $A^{-1}$ is bounded. In this section we confine our attention to the class of operators $A$ which has a bounded inverse $A^{-1}$ and satisfies (1.5). $\mu+A$ satisfies these conditions if $\mu>0$ and $A$ satisfies (1.5).

The distinctive feature of this case is that $(\lambda+A)^{-1}$ is regular at zero. In fact, (3.3) is satisfied for every $n$ by setting $y_{-k}=A^{-k} x$, $k=1, \cdots, n$. Thus it follows that $R^{o}=X$ and, as is easily seen, these spaces are isomorphic. (Conversely, we can prove that if $R^{\sigma}=X$ for a $\sigma>0$, then $A$ has a bounded inverse.)

In particular, $A_{-}^{\alpha} x$ is defined everywhere and analytic in $\alpha$ if $\operatorname{Re} \alpha<0$. Furthermore, if $x \in D^{\sigma}$, then $A^{\alpha} x$ is analytic in the halfplane $\operatorname{Re} \alpha<\sigma$.

The following proposition gives a useful representation of $A_{-}^{\alpha}$. This was used by Krasnosel'skii-Sobolevskii [13] to define $A^{\alpha}$ and $A^{-\alpha}$.

Proposition 5.1. Let $A$ have a continuous inverse and $n$ be a positive integer. Then for every $\alpha$ with $-(n+1)<\operatorname{Re} \alpha<0$ we have

$$
A_{-}^{\alpha}=-\frac{\sin \pi \alpha}{\pi} \frac{n !}{(\alpha+1) \cdots(\alpha+n)} \int_{0}^{\infty} \lambda^{\alpha+n}(\lambda+A)^{-n-1} d \lambda,
$$

where the integral converges in the uniform operator topology.

Proof. It is evident that the integral (5.1) converges in the uniform operator topology and represents an analytic function of $\alpha$ for $-(n+1)<\operatorname{Re} \alpha<0$. Integrating (5.1) by parts, we see that the integral does not depend on $n$. When $n=0,(5.1)$ coincides with (4.10). Thus it follows from the unique continuation property of analytic functions that (5.1) holds for $-(n+1)<\operatorname{Re} \alpha<0$.

We remark that $A_{0}^{\alpha}=A_{+}^{\alpha}$ for $\operatorname{Re} \alpha>0$, while $A_{0}^{\alpha}=\left.A_{-}^{\alpha}\right|_{\overline{D(A)}}$ for $\operatorname{Re} \alpha<0$ and that the latter operator is bounded in $\overline{D(A)}$. 
Proposition 5.2. If $\operatorname{Re} \alpha<0(\operatorname{Re} \alpha>0)$ and $x \in D\left(A_{0}^{\beta}\right)\left(x \in D\left(A_{0}^{\alpha+\beta}\right)\right)$, then $x \in D\left(A_{0}^{\alpha+\beta}\right)\left(x \in D\left(A_{0}^{\beta}\right), A_{0}^{\beta} x \in D\left(A_{0}^{\alpha}\right)\right.$, respectively) and

$$
A_{0}^{\alpha} A_{0}^{\beta} x=A_{0}^{\alpha+\beta} x .
$$

In particular, $D\left(A_{0}^{\alpha}\right) \subset D\left(A_{0}^{\beta}\right)$ if $\operatorname{Re} \alpha>\operatorname{Re} \beta$.

Proof. Let $\operatorname{Re} \alpha<0$. By Proposition 4.13, (5.2) holds for all $x \in D^{\sigma}$ if $\sigma$ is sufficiently large. If $x \in D\left(A_{0}^{\beta}\right)$, it can be approximated by a sequence $x_{j} \in D^{\sigma}$ in such a way that $x_{j} \rightarrow x$ and $A_{0}^{\beta} x_{j} \rightarrow A_{0}^{\beta} x$. Since $A_{0}^{\alpha}$ is bounded, $A_{0}^{\alpha} A_{0}^{\beta} x_{j}=A_{0}^{\alpha+\beta} x_{j}$ converges to $A_{0}^{\alpha} A_{0}^{\beta} x$. This proves by the closedness of $A_{0}^{\alpha+\beta}$ that $x \in D\left(A_{0}^{\alpha+\beta}\right)$ and $A_{0}^{\alpha+\beta} x=A_{0}^{\alpha} A_{0}^{\beta} x$. The proof in the case $\operatorname{Re} \alpha>0$ is similar.

Corollary 5.3. If $\operatorname{Re} \alpha>0$, then $A_{+}^{\alpha}=A_{0}^{\alpha}$ is the inverse of $A_{0}^{-\alpha}=\left.A_{-}^{-\alpha}\right|_{\overline{D(A)}}$. In particular the domain $D\left(A_{+}^{\alpha}\right)$ is contained in the range $R\left(A_{-}^{-\alpha}\right)$.

Proposition 5.4. Let $\operatorname{Re} \alpha>0$. If $\operatorname{Re} \alpha$ is not an integer, then the range $R\left(A_{-}^{-\alpha}\right)$ is contained in $D^{\mathrm{Ro} \alpha}$ and there exists a constant $K(\alpha)$ depending only on $M, L$ and $\alpha$ such that

$$
q_{\mathrm{Re} \alpha}\left(A_{-}^{-\alpha} x\right) \leqq K(\alpha)\|x\|, \quad x \in X .
$$

If $\alpha$ is an integer $n$, then $A_{-}^{-\alpha}=A^{-n}$, so that $R\left(A_{-\alpha}^{-\alpha}\right)=D\left(A^{n}\right)$. Finally, if $\operatorname{Re} \alpha$ is an integer $n$ and $\alpha \neq n$, then $R\left(A_{-}^{-\alpha}\right) \subset D\left(A^{n-1}\right)$ and there exists a constant $K_{1}(\alpha)$ depending only on $M, L,\left\|A^{-1}\right\|$ and $\alpha$ such that

$$
\begin{array}{r}
\left\|A^{n}(\lambda+A)^{-1} A^{-\alpha} x\right\| \leqq K_{1}(\alpha) \lambda^{-1} \log (\lambda+2)\|x\|, \\
x \in X, 0<\lambda<\infty .
\end{array}
$$

Consequently $R\left(A_{-\alpha}^{-\alpha}\right)$ is contained continuously in $D^{R_{e} \alpha-\varepsilon}$ for every $\varepsilon>0$.

Proof. Let $\alpha=n+\beta$, where $n=0,1,2, \cdots$ and $0<\operatorname{Re} \beta \leqq 1$. In view of Proposition 4.10, we can show that

$$
A_{-}^{-\alpha}=A^{-n} A_{-}^{-\beta}
$$

similarly to Proposition 5.2. Hence we have $R\left(A_{-}^{-\alpha}\right) \subset D\left(A^{n}\right)$ and

$$
A^{n} A_{-}^{-\alpha} x=A_{-}^{-\beta} x, \quad x \in X .
$$

Thus the problem may be reduced to the case $n=0$. The statement is obvious in the case $\beta=1$. Let $\beta \neq 1$. Then we have by Proposition 5.1 


$$
\frac{\pi(1-\beta)}{\sin \pi \beta} A(\lambda+A)^{-1} A_{-\beta}^{-\beta} x=\int_{0}^{\infty} \tau^{1-\beta} A(\lambda+A)^{-1}(\tau+A)^{-2} x d \tau .
$$

We split the integral into two parts and estimate each of them as follows:

$$
\begin{aligned}
& \left\|A(\lambda+A)^{-1} \int_{\lambda}^{\infty} \tau^{1-\beta}(\tau+A)^{-2} x d \tau\right\| \\
& \quad \leqq L M^{2} \int_{\lambda}^{\infty} \tau^{1-\operatorname{Re} \beta} \tau^{-2} d \tau\|x\| \\
& \quad \leqq L M^{2}(\operatorname{Re} \beta)^{-1} \lambda^{-\operatorname{Re} \beta}\|x\|, \\
& \left\|(\lambda+A)^{-1} \int_{0}^{\lambda}\left(A(\tau+A)^{-1}\right) \tau^{1-\beta}(\tau+A)^{-1} x d \tau\right\| \\
& \quad \leqq M \lambda^{-1} L M \int_{0}^{\lambda} \tau^{1-\operatorname{Re} \beta} \tau^{-1} d \tau\|x\| \\
& \quad \leqq L M^{2}(1-\operatorname{Re} \beta)^{-1} \lambda^{-\operatorname{Re} \beta}\|x\| \text { if } \operatorname{Re} \beta<1 .
\end{aligned}
$$

In case $\operatorname{Re} \beta=1$, the last integral becomes infinity. We note, however, that there is a constant $K$ such that $\left\|(\tau+A)^{-1}\right\| \leqq K(1+\tau)^{-1}$, $0<\tau<\infty$. Thus the last integral may be replaced by

$$
\begin{aligned}
& M \lambda^{-1} L K \int_{0}^{\lambda}(1+\tau)^{-1} d \tau\|x\| \\
& \quad=M L K \lambda^{-1} \log (1+\lambda)\|x\| .
\end{aligned}
$$

Proposition 5.5 (Krasnosel'skii-Sobolevskii [13]). Let $\alpha, \beta$ and $\gamma$ be complex numbers such that $\operatorname{Re} \alpha>\operatorname{Re} \gamma>\operatorname{Re} \beta$. Then we have

$$
\left\|A_{0}^{\lambda} x\right\| \leqq C(\alpha, \beta, \gamma)\left\|A_{0}^{\alpha} x\right\|^{\theta}\left\|A_{0}^{\beta} x\right\|^{1-\theta}, \quad x \in D\left(A_{0}^{\alpha}\right),
$$

where

$$
\theta=\operatorname{Re}(\gamma-\beta) / \operatorname{Re}(\alpha-\beta)
$$

in case $\operatorname{Re}(\alpha-\beta)$ is not an integer or $\alpha-\beta$ is an integer, and an arbitrary number greater than the right-hand side of (5.6) otherwise. The constant $C(\alpha, \beta, \gamma)$ depends only on $\alpha, \beta, \gamma$ and $\theta$.

Proof. In view of Proposition 5.2 we may assume that $\beta=0$. Then the statement is an immediate consequence of Propositions 5.4 and 4.7.

6. Domain $D\left(A^{\alpha}\right)$. We examine the domain $D\left(A_{+}^{\alpha}\right)\left(D\left(A_{-}^{\alpha}\right)\right)$ in connection with $D^{\sigma}(A)$ and $D\left((\mu+A)_{+}^{\alpha}\right), \mu>0\left(R^{o}(A)\right.$ and $D\left(\left(A(\mu+A)^{-1}\right)_{-}^{\alpha}\right)$, $\mu>0$, respectively). First we note

Proposition 6.1. $D^{\sigma}(\mu+A)$ is identical with and isomorphic to 
$D^{o}(A)$ for every $\sigma \geqq 0$ and every $\mu \geqq 0$.

We omit the proof which is straightforward.

Proposition 6.2. Let $\mu>0$ and $\nu \geqq 0$. Then the bounded operator $(\nu+A)(\mu+A)^{-1}$ satisfies (1.5). If $\operatorname{Re} \alpha>0$, then its fractional power $\left((\nu+A)(\mu+A)^{-1}\right)_{+}^{\alpha}$ is bounded and expressed as

$$
\left((\nu+A)(\mu+A)^{-1}\right)_{+}^{\alpha}=1-\frac{\sin \pi \alpha}{\pi} \int_{\nu}^{\mu}(\lambda-\nu)^{\alpha}(\mu-\lambda)^{-\alpha}(\lambda+A)^{-1} d \lambda,
$$

where the integral is taken in the sense of Sato. In terms of the convergent integral this is equal to

$$
\begin{aligned}
& 1+\alpha(\nu-\mu)(\mu+A)^{-1}+\cdots \\
& \quad+\frac{\alpha(\alpha-1) \cdots(\alpha-n+1)}{n !}(\nu-\mu)^{n}(\mu+A)^{-n} \\
& \quad-\frac{\sin \pi \alpha}{\pi} \int_{\nu}^{\mu}(\lambda-\nu)^{\alpha}(\mu-\lambda)^{-\alpha+n}(\lambda+A)^{-1}(\mu+A)^{-n} d \lambda,
\end{aligned}
$$

where $n$ is an integer such that $n+1>\operatorname{Re} \alpha$.

Proof. It is easy to see that if $\lambda>0$, then $\left(\lambda+(\nu+A)(\mu+A)^{-1}\right)$ is one-to-one and the inverse is given by

$$
(\lambda+1)^{-1}\left(1+(\mu-\nu)(\lambda+1)^{-1}\left(\lambda(\mu-\nu)(\lambda+1)^{-1}+\nu+A\right)^{-1}\right) .
$$

Thus $(\nu+A)(\mu+A)^{-1}$ satisfies condition (1.5). Since $(\nu+A)(\mu+A)^{-1}$ is bounded, it follows that $D^{\sigma}\left((\nu+A)(\mu+A)^{-1}\right)=X$ for all $\sigma \geqq 0$ and that they are isomorphic to each other. Therefore, $\left((\nu+A)(\mu+A)^{-1}\right)^{\alpha}$ is bounded for $\operatorname{Re} \alpha>0$ and analytic in $\alpha$. On the other hand, an easy calculation shows that expression (6.2) does not depend on $n$ as far as $0<\operatorname{Re} \alpha<n+1$ and represents a bounded operator valued analytic function of $\alpha$ for $\operatorname{Re} \alpha>0$. Thus it is enough to prove that (6.1) is true for $0<\alpha<1$. This can be done by expressing $\left((\nu+A)(\mu+A)^{-1}\right)_{+}^{\alpha}$ as integral (4.2) in terms of (6.3) and then by changing the variable as $\tau=(\lambda \mu+1) /(\lambda+1)$.

Proposition 6.3. Let $\operatorname{Re} \alpha>0, \mu>0$ and $\nu \geqq 0$. If $x \in \overline{D(A)}$, then $(\mu+A)_{0}^{-\alpha} x \in D\left((\nu+A)_{+}^{\alpha}\right)$ and

$$
(\nu+A)_{+}^{\alpha}(\mu+A)_{0}^{-\alpha} x=\left((\nu+A)(\mu+A)^{-1}\right)_{+}^{\alpha} x .
$$

If $x \in D\left((\nu+A)_{+}^{\alpha}\right)$, then

$$
\begin{aligned}
(\mu+A)_{0}^{-\alpha}(\nu+A)_{+}^{\alpha} x & =(\nu+A)_{+}^{\alpha}(\mu+A)_{0}^{-\alpha} x \\
& =\left((\nu+A)(\mu+A)^{-1}\right)_{+}^{\alpha} x .
\end{aligned}
$$


Proof. First we prove that (6.5) is valid for every $x \in D^{\sigma}$ if $\sigma$ is sufficiently large. In view of Proposition 5.1, we see easily that $(\mu+A)^{-\alpha}$ maps $D^{\sigma}$ into $D^{\sigma}$ for every $\sigma$ and that it commutes with $A^{k}$ and $(\lambda+A)^{-k}$. Therefore, we have

$$
(\mu+A)_{-}^{-\alpha}(\nu+A)_{+}^{\alpha} x=(\nu+A)_{+}^{\alpha}(\mu+A)_{-}^{-\alpha} x
$$

for every $x \in D^{\sigma}$ if $\sigma>\operatorname{Re} \alpha$.

Let $x \in D^{\sigma}, \sigma>0$. Then it follows that $(\mu+A)_{-}^{-\alpha}(\nu+A)_{+}^{\alpha} x$ is analytic for $0<\operatorname{Re} \alpha<\sigma$ since $(\mu+A)_{-}^{-\alpha}$ is analytic for $\operatorname{Re} \alpha>0$ and $(\nu+A)_{+}^{\alpha} x$ is analytic for $0<\operatorname{Re} \alpha<\sigma . \quad\left((\nu+A)(\mu+A)^{-1}\right)_{+}^{\alpha} x$ is also analytic for $\operatorname{Re} \alpha>0$, and expressed by integral (6.1). Therefore, to prove (6.4) for $x \in D^{\sigma}$, it is sufficient to show that $(\nu+A)^{\alpha}(\mu+A)^{-\alpha} x$ is equal to the integral (6.1) for $0<\alpha<1$ and all $x \in D(A)$. By (4.2) and (5.1) for $n=0$ we have

$$
\begin{aligned}
(\nu & +A)_{+}^{\alpha}(\mu+A)_{-}^{-\alpha} x \\
& =\left(\frac{\sin \pi \alpha}{\pi}\right)^{2} \int_{\nu}^{\infty}(\lambda-\nu)^{\alpha-1} d \lambda \int_{\mu}^{\infty}(\tau-\mu)^{-\alpha}(\lambda+A)^{-1}(\tau+A)^{-1}(\nu+A) x d \tau \\
& =\left(\frac{\sin \pi \alpha}{\pi}\right)^{2} \int_{\nu}^{\infty}(\lambda-\nu)^{\alpha-1} d \lambda \int_{\mu}^{\infty}(\tau-\mu)^{-\alpha} \frac{(\tau+A)^{-1}-(\lambda+A)^{-1}}{\lambda-\tau}(\nu+A) x d \tau .
\end{aligned}
$$

Now by a similar computation as in the proof of Proposition 4.9 we obtain

$$
\begin{aligned}
(\nu+A)_{+}^{\alpha}(\mu+A)_{-}^{-\alpha} x & \\
= & \frac{\sin \pi \alpha}{\pi}\left[\int_{\nu}^{\infty}(\lambda-\nu)^{\alpha-1}(\mu-\lambda-i 0)^{-\alpha}(\lambda+A)^{-1}(\nu+A) x d \lambda\right. \\
& \left.\quad+\int_{\mu}^{\infty}(\tau-\mu)^{-\alpha}(\nu-\tau+i 0)^{\alpha-1}(\tau+A)^{-1}(\nu+A) x d \lambda\right] \\
= & \frac{\sin \pi \alpha}{\pi} \int_{\nu}^{\mu}(\lambda-\nu)^{\alpha-1}(\mu-\lambda)^{-\alpha}(\lambda+A)^{-1}(\nu+A) x d \lambda \\
= & x-\frac{\sin \pi \alpha}{\pi} \int_{\nu}^{\mu}(\lambda-\nu)^{\alpha}(\mu-\lambda)^{-\alpha}(\lambda+A)^{-1} x d \lambda .
\end{aligned}
$$

Thus we have proved that (6.5) holds for every $x \in D^{\sigma}$ when $\sigma$ is large enough.

Hence the statements of the theorem follow by the same arguments as in the proof of Proposition 5.2.

TheOREM 6.4. If $\operatorname{Re} \alpha>0$, the domain $D\left((\nu+A)_{+}^{\alpha}\right)$ does not depend on $\nu \geqq 0$ and coincides with the range $R\left((\mu+A)_{0}^{-\alpha}\right)=R\left(\left.(\mu+A)_{-}^{-\alpha}\right|_{\overline{D(A)})}\right)$ for $\mu>0$.

Proof. It follows from Proposition 6.3 and Corollary 5.3 that if 
$\mu>0$ and $\nu \geqq 0$,

$$
D\left((\mu+A)_{+}^{\alpha}\right)=R\left((\mu+A)_{0}^{-\alpha}\right) \subset D\left((\nu+A)_{+}^{\alpha}\right) .
$$

Thus if $\mu$ and $\nu$ are positive, then we have $D\left((\mu+A)_{+}^{\alpha}\right)=D\left((\nu+A)_{+}^{\alpha}\right)$ and $D\left((\mu+A)_{+}^{\alpha}\right)$ is contained in $D\left(A_{+}^{\alpha}\right)$.

To prove that $D\left(A_{+}^{\alpha}\right) \subset D\left((\mu+A)_{+}^{\alpha}\right)$, we show that the restriction of $A_{+}^{\alpha}$ to $D\left((\mu+A)_{+}^{\alpha}\right)$ is closed in $X$. Proposition 6.3 gives

$$
\begin{aligned}
& \left(A_{+}^{\alpha}-(\mu+A)_{+}^{\alpha}\right)(\mu+A)_{0}^{-\alpha} x=\alpha(-\mu)(\mu+A)^{-1} x+\cdots \\
& \quad+\frac{\alpha(\alpha-1) \cdots(\alpha-n+1)}{n !}(-\mu)^{n}(\mu+A)^{-n} x \\
& -\frac{\sin \pi \alpha}{\pi} \int_{0}^{\mu} \lambda^{\alpha}(\mu-\lambda)^{-\alpha+n}(\lambda+A)^{-1}(\mu+A)^{-n} x d \lambda
\end{aligned}
$$

for every $x \in \overline{D(A)}$. We notice that the right-hand side remains bounded if we operate $\mu+A$ on both sides. Therefore, there is a constant $C$ such that

$$
\left\|(\mu+A)\left(A_{+}^{\alpha}-(\mu+A)_{+}^{\alpha}\right) x\right\| \leqq C\left\|(\mu+A)_{+}^{\alpha} x\right\|, \quad x \in D\left((\mu+A)_{+}^{\alpha}\right) .
$$

Let $x=(\mu+A)^{-1} y$ with $y \in D\left((\mu+A)_{+}^{\alpha}\right)$ and use Propositions 4.4, 5.2 and 5.5. Then we obtain the estimate

$$
\begin{aligned}
\left\|\left(A_{+}^{\alpha}-(\mu+A)_{+}^{\alpha}\right) y\right\| & \leqq C\left\|(\mu+A)_{0}^{\alpha-1} y\right\| \\
& \leqq \frac{1}{2}\left\|(\mu+A)_{+}^{\alpha} y\right\| \quad C_{1}\|y\|
\end{aligned}
$$

with a constant $C_{1}$ independent of $y \in D\left((\mu+A)_{+}^{\alpha}\right)$. Hence it follows that

$$
\left\|(\mu+A)_{+}^{\alpha} x\right\| \leqq 2\left\|A_{+}^{\alpha} x\right\|+2 C_{1}\|x\|, \quad x \in D\left((\mu+A)_{+}^{\alpha}\right) .
$$

Now it is easy to see that $A_{+}^{\alpha}$ restricted to $D\left((\mu+A)_{+}^{\alpha}\right)$ is closed.

TheOREM 6.5. Let $\operatorname{Re} \alpha>0$. If $\alpha$ is real or $\operatorname{Re} \alpha$ is not an integer, then $D\left(A_{+}^{\alpha}\right)$ is contained in the space $D^{\mathrm{Re} \alpha}$. There is a constant $K(\alpha)$ depending only on $M, L$ and $\alpha$ such that

$$
q_{\mathrm{Re} \alpha}(x) \leqq K(\alpha)\left\|A_{+}^{\alpha} x\right\|, \quad x \in D\left(A_{+}^{\alpha}\right) .
$$

Thus the embedding map is continuous. Even when $\operatorname{Re} \alpha$ is an integer, $D\left(A_{+}^{\alpha}\right)$ is contained in every $D^{\mathrm{Re} \alpha-z}$ for $\varepsilon>0$ continuously. In particular, we have $D\left(A_{+}^{\alpha}\right) \subset D\left(A_{+}^{\beta}\right)$ if $0<\operatorname{Re} \beta<\operatorname{Re} \alpha$.

Proof. The statements except (6.6) are immediate consequences of Theorem 6.4 and Proposition 5.4. Let $\operatorname{Re} \alpha=n+\rho, 0<\rho<1$. Then $(6.6)$ is equivalent to 
(6.7)

$$
\left\|A^{n+1}(\lambda+A)^{-1} x\right\| \leqq K(\alpha) \lambda^{-\rho}\left\|A_{+}^{\alpha} x\right\|, \quad \lambda>0, \quad x \in D\left(A_{+}^{\alpha}\right) .
$$

It follows from Proposition 5.4 that if $\nu>0$,

$$
\left\|(\nu+A)^{n+1}(\lambda+\nu+A)^{-1} x\right\| \leqq K(\alpha) \lambda^{-\rho}\left\|(\nu+A)_{+}^{\alpha} x\right\|
$$

for every $x \in D\left(A_{+}^{\alpha}\right)$, where $K(\alpha)$ does not depend on $\nu$. Let $\nu$ tend to 0 with $\lambda$ fixed. Then clearly the left-hand side converges to that of (6.7). Further, it follows from Propositions 6.2 and 6.3 that $(\nu+A)_{+}^{\alpha}(\mu+A)_{-}^{-\alpha}$ converges to $A_{+}^{\alpha}(\mu+A)_{-}^{-\alpha}$ in the uniform operator topology of $\overline{D(A)}$. Thus we have $(\nu+A)_{+}^{\alpha} x \rightarrow A_{+}^{\alpha} x$ for every $x \in D\left(A_{+}^{\alpha}\right)$. This proves (6.7) and the proof is completed.

In view of Theorem 3.2 and in particular identity (3.8) the discussions concerning the domain $D\left(A_{-}^{\alpha}\right)$ may be reduced to the case considered above. We summarize the results in the following propositions and theorems.

Proposition 6.6. $R^{\sigma}(A)$ is identical with and isomorphic to. $R^{o}\left(A(\mu+A)^{-1}\right)$ for every $\sigma \geqq 0$ and every $\mu>0$.

Proposition 6.7. Let $\operatorname{Re} \alpha<0$ and $\mu>0$. If $x \in \overline{R(A)}$, then $\left(A(\mu+A)^{-1}\right)_{0}^{-\alpha} x \in D\left(A_{-}^{\alpha}\right)$ and we have

$$
A_{-}^{\alpha}\left(A(\mu+A)^{-1}\right)_{0}^{-\alpha} x=(\mu+A)_{0}^{\alpha} x .
$$

If $x \in D\left(A^{\alpha}\right)$, then it follows that

$$
A_{-}^{\alpha}\left(A(\mu+A)^{-1}\right)_{0}^{-\alpha} x=\left(A(\mu+A)^{-1}\right)_{0}^{-\alpha} A_{-\alpha}^{\alpha} x=(\mu+A)_{0}^{\alpha} x .
$$

THEOREM 6.8. If $\operatorname{Re} \alpha<0$ and $\mu>0$, we have

$$
D\left(A_{-}^{\alpha}\right)=D\left(\left(A(\mu+A)^{-1}\right)_{-}^{\alpha}\right)=R\left(\left.\left(A(\mu+A)^{-1}\right)_{+}^{-\alpha}\right|_{\overline{R(A)}}\right) .
$$

THeOREM 6.9. If $\operatorname{Re} \alpha<0$ and if $\alpha$ is real or $\operatorname{Re} \alpha$ is not an. integer, then we have $D\left(A^{\alpha}\right) \subset R^{-\mathrm{Re} \alpha}$, and the embedding map is continuous. When $\operatorname{Re} \alpha$ is an integer, $D\left(A_{-}^{\alpha}\right)$ is not necessarily contained in $R^{-\mathrm{Re} \alpha}$, but it is contained in $R^{-\mathrm{Re} \alpha-\varepsilon}$ for any $\varepsilon>0$ and the embedding map is continuous. Thus we have $D\left(A_{-}^{\alpha}\right) \subset D\left(A_{-}^{\beta}\right)$ if $\operatorname{Re} \alpha<\operatorname{Re} \beta$.

\section{Additivity and range.}

Theorem 7.1. If $\operatorname{Re} \alpha \cdot \operatorname{Re} \beta>0$, then we have

$$
A_{ \pm}^{\alpha} A_{ \pm}^{\beta}=A_{0}^{\alpha} A_{ \pm}^{\beta}=A_{ \pm}^{\alpha+\beta}
$$

in the sense of the product of operators. 
Proof. It is sufficient to consider the case $\operatorname{Re} \alpha>0$ and $\operatorname{Re} \beta>0$. "The case $\operatorname{Re} \alpha<0$ may be reduced to this case. We know that if $\sigma$ is sufficiently large,

$$
A_{+}^{\alpha} A_{+}^{\beta} x=A_{+}^{\alpha+\beta} x
$$

holds for every $x \in D^{\sigma}$. Theorem 6.5 shows $D\left(A_{+}^{\alpha+\beta}\right) \subset D\left(A_{+}^{\beta}\right)$. Thus there is a constant $C$ such that

$$
\left\|A_{+}^{\beta} x\right\| \leqq C\left(\left\|A_{+}^{\alpha+\beta} x\right\|+\|x\|\right), \quad x \in D\left(A_{+}^{\alpha+\beta}\right) .
$$

Now let $x \in D\left(A_{+}^{\alpha+\beta}\right)$. Then there exists a sequence $x_{j} \in D^{\sigma}$ such that $x_{j} \rightarrow x$ and $A_{+}^{\alpha+\beta} x_{j} \rightarrow A_{+}^{\alpha+\beta} x$. Hence we have, by (7.2) and (7.3), $A_{+}^{\beta} x_{j} \rightarrow A_{+}^{\beta} x$ and $A_{+}^{\alpha} A_{+}^{\beta} x_{j} \rightarrow A_{+}^{\alpha+\beta} x$. This proves $A_{+}^{\beta} x \in D\left(A_{+}^{\alpha}\right)$ and $A_{+}^{\alpha} A_{+}^{\beta} x=A_{+}^{\alpha+\beta} x$. Since $A^{\beta} x \in \overline{R(A)}$, it follows that $A_{+}^{\alpha} A_{+}^{\beta} x=A_{0}^{\alpha} A_{+}^{\beta} x$.

Conversely let $x \in D\left(A_{+}^{\alpha} A_{+}^{\beta}\right)$. If $l$ is a sufficiently large integer, we have $x^{\mu}=\left(\mu(\mu+A)^{-1}\right)^{l} \in D^{\sigma}$ and $x^{\mu} \rightarrow x$ as $\mu \rightarrow \infty$. From (7.2) for $x \in D^{\sigma}$ and Proposition 4.4 it follows that

$$
\begin{aligned}
A_{+}^{\alpha+\beta} x^{\mu} & =A_{+}^{\alpha} A_{+}^{\beta}\left(\mu(\mu+A)^{-1}\right)^{l} x \\
& =\left(\mu(\mu+A)^{-1}\right)^{l} A_{+}^{\alpha} A_{+}^{\beta} x \\
& \rightarrow A_{+}^{\alpha} A_{+}^{\beta} x \text { as } \mu \rightarrow \infty .
\end{aligned}
$$

Hence we have $x \in D\left(A_{+}^{\alpha+\beta}\right)$ and $A_{+}^{\alpha+\beta} x=A_{+}^{\alpha} A_{+}^{\beta} x$.

If we use $\left(\mu(\mu+A)^{-1}\right)^{l}\left(A(\nu+A)^{-1}\right)^{h}$ instead of $\left(\mu(\mu+A)^{-1}\right)^{l}$, we can prove that

$$
A_{0}^{\alpha} A_{0}^{\beta} \subset A_{0}^{\alpha+\beta}
$$

for all $\alpha$ and $\beta$. On the other hand, we hnow that if $\sigma$ and $\tau$ are large enough, $D^{\sigma} \cap R^{\tau}$ is contained both in $D\left(A_{0}^{\alpha} A_{0}^{\beta}\right)$ and in $D\left(A_{0}^{\alpha+\beta}\right)$, and $A_{0}^{\alpha+\beta}$ is the smallest closed extension of its restriction to $D^{\sigma} \cap R^{\tau}$. Thus we obtain the following theorem.

Theorem 7.2. For arbitrary $\alpha$ and $\beta$ we have

$$
\left[A_{0}^{\alpha} A_{0}^{\beta}\right]_{\sigma}=A_{0}^{\alpha+\beta},
$$

where $\left[A_{0}^{\alpha} A_{0}^{\beta}\right]_{o}$ denotes the smallest closed extension of $A_{0}^{\alpha} A_{0}^{\beta}$.

More precisely the following theorem holds.

THEOREM 7.3. If $x \in D\left(A_{0}^{\beta}\right) \cap D\left(A_{0}^{\alpha+\beta}\right)$, then $A_{0}^{\beta} x \in D\left(A_{0}^{\alpha}\right)$ and satisfies

$$
A_{0}^{\alpha} A_{0}^{\beta} x=A_{0}^{\alpha+\beta} x .
$$

In particular, $A_{0}^{\alpha}$ is one-to-one for every $\alpha$ and the inverse is the same as $A_{0}^{-\alpha}$. 
Proof. Let $x^{\mu \nu}=\left(\mu(\mu+A)^{-1}\right)^{l}\left(A(\nu+A)^{-1}\right)^{h}$ with $l$ and $h$ sufficiently large. Then it follows that $x^{\mu \nu} \rightarrow x, A_{0}^{\beta} x^{\mu \nu} \rightarrow A_{0}^{\beta} x$ and $A_{0}^{\alpha+\beta} x^{\mu \nu}=A_{0}^{\alpha}$. $A_{0}^{\beta} x^{\mu \nu} \rightarrow A_{0}^{\alpha+\beta} x$ as $\mu \rightarrow \infty$ and $\nu \rightarrow 0$. Since $A_{0}^{\alpha}$ is closed, we have thus. the statement of the theorem.

CoROLlary 7.4. If $A$ has $a$ bounded inverse and if $\operatorname{Re} \alpha>0$, then

$$
A_{0}^{\alpha} A_{0}^{\beta}=A_{0}^{\alpha+\beta}
$$

Proof. By Proposition 5.2, $D\left(A_{0}^{\beta}\right) \cap D\left(A_{0}^{\alpha+\beta}\right)=D\left(A_{0}^{\alpha+\beta}\right)$.

The results obtained above give us information about the ranges. of operators $A_{ \pm}^{\alpha}$ and $A_{0}^{\alpha}$ restriction to certain subspaces. We give here only one proposition as an illustration.

Proposition 7.5. Let $\operatorname{Re} \alpha>\operatorname{Re} \beta>0$. Then

$$
A_{+}^{\beta}\left(D\left(A_{+}^{\alpha}\right)\right) \subset D\left(A_{0}^{\gamma}\right)
$$

if $\gamma=\alpha-\beta$ or $-\operatorname{Re} \beta<\operatorname{Re} \gamma<\operatorname{Re}(\alpha-\beta)$.

Proof. It follows from Theorem 7.1 that

$$
A_{+}^{\alpha}=A_{0}^{\alpha-\beta} A_{+}^{\beta} \text {. }
$$

This proves the proposition for $\gamma=\alpha-\beta$. Similarly we have

$$
A_{+}^{\beta}=A_{0}^{-\gamma} A_{+}^{\beta+\gamma}
$$

for $0>\operatorname{Re} \gamma>-\operatorname{Re} \beta$. Hence Theorem 7.3 shows that $R\left(A_{+}^{\beta}\right) \subset D\left(A_{0}^{\gamma}\right)$ 。 Now the proposition for a general $\gamma$ follows from Proposition 4.12 and Theorem 6.5.

In this connection the following theorem will be interesting.

THEOREM 7.6. (i) Let $\sigma>\operatorname{Re} \alpha>0$ and let $\sigma-\operatorname{Re} \alpha$ be not an. integer. Then $A_{+}^{\alpha}$ maps $D^{\sigma}$ into $D^{\sigma-\mathrm{Re} \alpha}$. (ii) Similarly let $\sigma \geqq 0$, $\operatorname{Re} \alpha<0$ and let $\sigma-\operatorname{Re} \alpha$ be not an integer. Then $A_{-}^{\alpha}$ maps $D^{\sigma} \cap D\left(A_{-}^{\alpha}\right)$ into $D^{\sigma-\mathrm{Re} \alpha}$.

Proof. (i) We may assume that $0<\operatorname{Re} \alpha<1$ without loss of generality. First we consider the case $\operatorname{Re} \alpha<\rho$. It is clear from the foregoing discussion that $A_{+}^{\alpha} x \in D\left(A^{n}\right)$. Using (4.2) and the commutativity of operators, $A, A_{+}^{\alpha}$ and $(\lambda+A)^{-1}$, we obtain

$$
\begin{aligned}
A^{n+1}(\lambda+A)^{-1} A_{+}^{\alpha} x & =\frac{\sin \pi \alpha}{\pi} \int_{0}^{\infty} \tau^{\alpha-1} A(\lambda+A)^{-1} A(\tau+A)^{-1} A^{n} x d \tau \\
& =\sin \pi \alpha / \pi\left(I_{1}+I_{2}\right),
\end{aligned}
$$


where $I_{1}$ and $I_{2}$ denote the integrals over $(0, \lambda)$ and $(\lambda, \infty)$, respectively. Since

$$
\begin{aligned}
\left\|A(\lambda+A)^{-1} A(\tau+A)^{-1} A^{n} x\right\| & \leqq\left\|A(\tau+A)^{-1}\right\|\left\|A^{n+1}(\lambda+A)^{-1} x\right\| \\
& \leqq L q_{\sigma}(x) \lambda^{-\rho}
\end{aligned}
$$

we have

$$
\left\|I_{1}\right\| \leqq L q_{o}(x)(\operatorname{Re} \alpha)^{-1} \lambda^{\operatorname{Re} \alpha-\rho} .
$$

In the same way we have

$$
\begin{aligned}
\left\|I_{2}\right\| & \leqq L \int_{\lambda}^{\infty}\left|\tau^{\alpha-1}\right| q_{\sigma}(x) \tau^{-\rho} d \tau \\
& =L q_{\sigma}(x)(\operatorname{Re} \alpha-\rho)^{-1} \lambda^{\operatorname{Re} \alpha-\rho} .
\end{aligned}
$$

Thus $A_{+}^{\alpha} x$ belongs to $D^{\sigma-\operatorname{Re} \alpha}$.

In the case $\operatorname{Re} \alpha>\rho$, it is enough to make use of the estimates

$$
\begin{aligned}
& \left\|A(\lambda+A)^{-1} A(\tau+A)^{-1} A^{n-1} x\right\| \leqq M q_{\sigma}(x) \lambda^{-1} \tau^{-\rho} \\
& \left\|A(\lambda+A)^{-1} A(\tau+A)^{-1} A^{n-1} x\right\| \leqq M q_{\sigma}(x) \lambda^{-\rho} \tau^{-1}
\end{aligned}
$$

for $I_{1}$ and $I_{2}$, respectively.

(ii) We may restrict ourselves to the case $0>\operatorname{Re} \alpha>-1$ as above. First, let $\rho-\operatorname{Re} \alpha<1$. Since $D^{\sigma} \cap D\left(A_{-}^{\alpha}\right)$ is contained in $\overline{D(A)} \cap \overline{R(A)}$, $A_{-}^{\alpha} x=A_{0}^{\alpha} x$ and $A^{n} x=A_{0}^{n} x$ hold for $x \in D^{\sigma} \cap D\left(A_{-}^{\alpha}\right)$. Hence we have by Theorem 7.3 $A_{-}^{\alpha} x \in D^{n}$ and

$$
\begin{aligned}
A^{n+1}(\lambda+A)^{-1} A_{-}^{\alpha} x & =A_{0}^{1+\alpha}(\lambda+A)^{-1} A^{n} x \\
& =-\frac{\sin \pi \alpha}{\pi} \int_{0}^{\infty} \tau^{\alpha} A(\lambda+A)^{-1}(\tau+A)^{-1} A^{n} x d \tau .
\end{aligned}
$$

In a similar way to (i), employing inequalities (7.10), we obtain

$$
A^{n+1}(\lambda+A)^{-1} A_{-\alpha}^{\alpha}=O\left(\lambda^{\operatorname{Re} \alpha-\rho}\right), \quad \lambda \rightarrow \infty .
$$

If $\rho-\operatorname{Re} \alpha>1$, we can apply $A$ to (7.11) under the integral sign, obtaining

$$
A^{n+2}(\lambda+A)^{-1} A^{\alpha} x=-\frac{\sin \pi \alpha}{\pi} \int_{0}^{\infty} \tau^{\alpha} A(\lambda+A)^{-1} A(\tau+A)^{-1} A^{n} x d \tau .
$$

From (7.8) and a similar inequality it follows that

$$
A^{n+2}(\lambda+A)^{-1} A_{-}^{\alpha} x=O\left(\lambda^{\operatorname{Re} \alpha-\rho+1}\right), \quad \lambda \rightarrow \infty .
$$

8. Convexity and continuity in $\alpha$.

THEOREM 8.1. Let $\operatorname{Re} \alpha>\operatorname{Re} \gamma>\operatorname{Re} \beta$. Then there is a constant 
$C(\alpha, \beta, \gamma)$ depending only on $M, L, \alpha, \beta, \arg (\alpha-\gamma)$, and $\arg (\gamma-\beta)$, such that

$$
\left\|A_{*}^{\gamma} x\right\| \leqq C(\alpha, \beta, \gamma)\left\|A_{*}^{\alpha} x\right\|^{\theta}\left\|A_{*}^{\beta} x\right\|^{1-\theta}, \quad x \in D\left(A_{*}^{\alpha}\right) \cap D\left(A_{*}^{\beta}\right),
$$

where $\theta=\operatorname{Re}(\gamma-\beta) / \operatorname{Re}(\alpha-\beta)$. Subscripts for $A_{*}^{\alpha}, A_{*}^{\beta}$ and $A_{*}^{\gamma}$ are assumed to be the same.

Proof. If $\operatorname{Re} \alpha \cdot \operatorname{Re} \beta \leqq 0, A$ may be replaced by $A_{B}$, so that we have $A_{*}^{\alpha} x=A_{0}^{\alpha-\beta} A_{0}^{\beta} x$, and $A_{*}^{\gamma} x=A_{0}^{\gamma-\beta} A_{0}^{\beta} x$. If $\operatorname{Re} \beta>0$, then by Theorem 7.1 we have also $A_{*}^{\alpha} x=A_{0}^{\alpha-\beta} A_{+}^{\beta} x$ and $A_{*}^{\gamma} x=A_{0}^{\gamma-\beta} A_{+}^{\beta} x$. Lastly the case $\operatorname{Re} \alpha<0$ is reduced to the above case by considering $A_{R}^{-1}$ instead of $A$. Thus we may assume that $\beta=0$ and $A=A_{B}$ without loss of generality.

If $\operatorname{Re} \alpha$ is not an integer, then it follows from Theorem 6.5 and (4.7) that

$$
\left\|A_{+}^{\gamma} x\right\| \leqq C(\alpha)\left[\frac{|\gamma|}{\operatorname{Re} \gamma} N^{\operatorname{Re} \gamma}\|x\|+\frac{C_{1}(\alpha)}{\operatorname{Re}(\alpha-\gamma)} N^{\operatorname{Re}(\gamma-\alpha)}\left\|A_{+}^{\alpha} x\right\|\right]
$$

for all $N>0$. Thus taking the minimum of the right-hand side, we obtain (8.1) with $C(\alpha, \beta, \gamma)$ depending also on $\operatorname{Re}(\alpha-\gamma)$. We may, however, change the roles of $\alpha$ and $\beta$ by Theorem 7.3. Therefore, taking $\min \{C(\alpha, \beta, \gamma), C(\beta, \alpha, \gamma)\}$ for $C(\alpha, \beta, \gamma)$, we have the assertion.

If $\operatorname{Re} \alpha$ is an integer, choose $\delta_{1}$ and $\delta_{2}$ such that $0<\delta_{1}<\delta_{2}<\operatorname{Re} \alpha$ and that any one of $\delta_{1}, \delta_{2}, \operatorname{Re} \alpha-\delta_{1}$ and $\operatorname{Re} \alpha-\delta_{2}$ is not an integer. Applying the same method as in the proof of Theorem 2.3, we obtain (8.1) for $\gamma=\delta_{1}$ and $\delta_{2}$. If we use these inequalities and (8.1) for $\alpha=\delta_{2}$ or for $\beta=\delta_{1}$, we can similarly prove the statement of the theorem.

Theorem 8.2. Let $x \in D\left(A_{*}^{\alpha}\right) \cap D\left(A_{*}^{\beta}\right), \operatorname{Re} \alpha>\operatorname{Re} \beta$. Then $A_{*}^{\gamma} x$ is analytic in the strip $\operatorname{Re} \beta<\operatorname{Re} \gamma<\operatorname{Re} \alpha$, and it converges to $A_{*}^{\alpha} x$ $\left(A_{*}^{\beta} x\right)$ as $\gamma$ tends to $\alpha(\beta)$ in a Stolz region $\arg (\alpha-\gamma) \leqq \theta<\pi / 2$ $(\arg (\gamma-\beta) \leqq \theta<\pi / 2$, respectively $)$.

Proof. The analyticity is already known. Theorem 8.1 implies that the operator $A_{*}^{\gamma}$ from the space $D\left(A_{*}^{\alpha}\right) \cap D\left(A_{*}^{\beta}\right)$ normed with $\|x\|+$ $\left\|A_{*}^{\alpha} x\right\|+\left\|A_{*}^{\beta}\right\|$ into $X$ is uniformly bounded if $\gamma$ is in a fixed Stolz region. If $x \in D^{\sigma}$ with $\sigma$ sufficiently large (or $D^{\sigma} \cap R^{\sigma}$ or $R^{\sigma}$ according as $\operatorname{Re} \beta>0$ or $\operatorname{Re} \alpha \cdot \operatorname{Re} \beta \leqq 0$ or $\operatorname{Re} \alpha<0)$, then $A_{*}^{\gamma} x$ is analytic at $\alpha$ and $\beta$. Since $D^{\sigma}$ (or $D^{\sigma} \cap R^{\sigma}$ or $R^{\sigma}$ ) is dense in $D\left(A_{*}^{\alpha}\right) \cap D\left(A_{*}^{\beta}\right)$, the statement follows from the Banach-Steinhaus theorem.

Proposition 8.3. Let $x \in D\left(A_{+}^{\alpha}\right)$ for an $\alpha$. Then $A_{+}^{\gamma} x$ converges to $x$ as $\gamma \rightarrow 0$ in a Stolz region if and only if $x \in \overline{R(A)}$. 
Proof. If $x \in \overline{R(A)}$, then it follows that $x \in D\left(A_{0}^{0}\right) \cap D\left(A_{0}^{\alpha}\right)$. Thus $A_{+}^{\gamma} x$ converges to $x$ as $\gamma \rightarrow 0$. Conversely if $A_{+}^{\gamma} x \rightarrow x$, then $x$ belongs to $\overline{R(A)}$ as the limit of elements in $\overline{R(A)}$.

Proposition 8.4. Let $\operatorname{Re} \alpha>0$ and $\gamma_{j}$ be a sequence such that $\gamma_{j} \rightarrow \alpha$ in a Stolz region $\arg \left(\alpha-\gamma_{j}\right) \leqq \theta<\pi / 2$. Then $x \in D\left(A_{+}^{\alpha}\right)$ if and only if $x \in D\left(A_{+}^{\gamma_{j}}\right)$ for all $j$ and $A_{+}^{\gamma_{j}} x$ converges (weakly).

Proof. Necessity is clear from Theorem 8.2. Let $A_{+}^{\gamma_{j}} x$ converge weakly to an element $y$. Since $x \in D^{\operatorname{Re} \alpha-z}$ for $\varepsilon>0$, we have $\left(\mu(\mu+A)^{-1}\right) x \in D\left(A_{+}^{\alpha}\right), \mu>0$. Thus it follows from the necessity part and Proposition 4.4 that

$$
\begin{aligned}
A_{+}^{\alpha}\left(\mu(\mu+A)^{-1}\right) x & =\lim A_{+}^{\gamma_{j}}\left(\mu(\mu+A)^{-1}\right) x \\
& =\mathrm{w}-\lim \left(\mu(\mu+A)^{-1}\right) A_{+}^{\gamma_{j}} x \\
& =\left(\mu(\mu+A)^{-1}\right) y .
\end{aligned}
$$

Let $\mu \rightarrow \infty$. Then the right-hand side tends to $y$ because $y$ is in $\overline{D(A)}$. Therefore, we have by Proposition $4.5 x \in D\left(A_{+}^{\alpha}\right)$ and $A_{+}^{\alpha} x=y$.

9. Domination. The results of this section have been announced by Krasnosel'skii-Sobolevskii [13].

Proposition 9.1. If $B$ is a closed linear operator from $X$ to another Banach space $Y$ such that the domain $D(B)$ contains $D\left(A_{+}^{\alpha}\right)$, then for any $\beta$ with $\operatorname{Re} \beta>\operatorname{Re} \alpha$, there is a constant $C$ such that

$$
\|B x\| \leqq C\left(\left\|A_{+}^{\beta} x\right\|+\|x\|\right)^{\theta}\|x\|^{1-\theta}, \quad x \in D\left(A_{+}^{\beta}\right),
$$

where $\theta=\operatorname{Re} \alpha / \operatorname{Re} \beta$.

Proof. It follows from the closed graph theorem that there is a constant $C_{1}$ such that

$$
\|B x\| \leqq C_{1}\left(\left\|A_{+}^{\alpha} x\right\|+\|x\|\right), \quad x \in D\left(A_{+}^{\alpha}\right) .
$$

(9.1) is an easy consequence of this inequality and (8.1).

THEOREM 9.2. If a closed linear operator $B$ satisfies (9.1) for a $\beta, \operatorname{Re} \beta>0$, and a $\theta, 0<\theta<1$, then $D(B)$ contains $D\left(A_{+}^{\alpha}\right)$ for every $\alpha$ with $\operatorname{Re} \alpha>\theta \operatorname{Re} \beta$.

Proof. We are concerned only with the domain $D\left(A_{+}^{\alpha}\right)$, so that we may replace $A$ by $A+1$ and assume that $A$ has a bounded inverse without loss of generality. Then $\left\|A_{+}^{\alpha} x\right\|$ gives an equivalent norm to 
$\left\|A_{+}^{\alpha} x\right\|+\|x\|$ in $D\left(A_{+}^{\alpha}\right)$. Further we may assume that $\beta$ in (9.1) is a positive integer $m$, because if (9.1) is valid for a $\beta$, then it follows from Theorem 8.1 that (9.1) holds for every $\beta$ with a greater real part without changing the value $\theta \operatorname{Re} \beta$.

It is plain from (1.5) and (1.6) that

$$
\left\|B(\lambda+A)^{-m} x\right\| \leqq C L^{m \theta} M^{m(1-\theta)}(\lambda+1)^{-m(1-\theta)}\|x\|, \quad x \in \overline{D(A)} .
$$

This implies by Proposition 5.1 that for any $\operatorname{Re} \alpha>m \theta$ there is a constant $C_{1}$ such that

$$
\left\|B A_{-}^{-\alpha} x\right\| \leqq C_{1}\|x\|, \quad x \in \overline{D(A)} .
$$

Since $A_{+}^{\alpha}$ is equal to $\left.\left(A_{-}^{-\alpha}\right)^{-1}\right|_{D(A)}$, we have (9.2), proving that $D(B) \supset D\left(A_{+}^{\alpha}\right)$.

Proposition 9.3. Let $A$ and $B$ be operators satisfying (1.5) and let $D\left(B_{+}^{\beta}\right) \supset D\left(A_{+}^{\alpha}\right)$ for an $\alpha$ and a $\beta$. Then we have $D\left(B_{+}^{\delta}\right) \supset D\left(A_{+}^{\gamma}\right)$ and

$$
\left\|B_{+}^{\delta} x\right\| \leqq C\left(\left\|A_{+}^{\gamma} x\right\|+\|x\|\right), \quad x \in D\left(A_{+}^{\gamma}\right)
$$

for any $\gamma$ and $\delta$ such that $0<\operatorname{Re} \delta<\operatorname{Re} \beta$ and $\operatorname{Re} \delta / \operatorname{Re} \beta<\operatorname{Re} \gamma / \operatorname{Re} \alpha$.

Proof. It follows from Theorem 8.1 that

$$
\begin{aligned}
\left\|B_{+}^{\delta} x\right\| & \leqq C\left\|B_{+}^{\beta} x\right\|^{\operatorname{Re} \delta / \operatorname{Re} \beta}\|x\|^{1-\operatorname{Re} \delta / \operatorname{Re} \beta} \\
& \leqq C_{1}\left(\left\|A_{+}^{\alpha} x\right\|+\|x\|\right)^{\operatorname{Re} \delta / \operatorname{Re} \beta}\|x\|^{1-\operatorname{Re} \delta / \operatorname{Re} \beta} .
\end{aligned}
$$

Now the statement follows from Theorem 9.2.

10. Resolvent and multiplicativity. This section is devoted to the resolvent of $A_{+}^{\alpha}$. We note that $A_{+}^{\alpha}$ is an operator in $\overline{D(A)}$, and hence the resolvent is considered only in $\overline{D(A)}$. It has been shown, however, that $A_{+}^{\alpha}$ is determined by its restriction $A_{D}$ which has a dense domain in $\overline{D(A)}$. Therefore, we may assume that $D(A)$ is dense in $X$. Further we shall restrict ourselves to the case $\alpha>0$, because this is the only case important in application. For the general spectral mapping theorem the reader is referred to Balakrishnan [2].

First we need a specification of the operator $A$. From the assumption (1.5) and the resolvent equation it follows easily that $(\lambda+A)^{-1}$ exists at least for $\lambda$ in the sector $|\arg \lambda|<\arcsin \left(M^{-1}\right)$ and that $\lambda(\lambda+A)^{-1}$ is uniformly bounded on each ray in the sector. Write

$$
M(\theta)=\sup _{|\arg \gamma|=\theta}\left\|\lambda(\lambda+A)^{-1}\right\|, \quad \theta \geqq 0 .
$$

Then by the Phragmén-Lindelöf theorem $M(\theta)$ is an increasing function of $\theta$ and satisfies some convexity property. 
Modifying the definition of Kato [10], we call an operator $A$ of type $(\omega,(M(\theta)), 0 \leqq \omega<\pi$, if $A$ is a densely defined closed linear operator such that the resolvent set of $-A$ contains the sector $|\arg \lambda|<\mu-\omega$, and

$$
\sup _{|\arg \lambda|=\theta}\left\|\lambda(\lambda+A)^{-1}\right\| \leqq M(\theta)<\infty
$$

holds for $0 \leqq \theta<\pi-\omega$. An operator $A$ is of type $(\omega, M(\theta))$ for an $\omega<\pi / 2$ if and only if $-A$ generates a semi-group $T_{t}$ which has an analytic extension to the sector $|\arg t|<\pi / 2-\omega$ such that the extended $T_{t}$ is uniformly bounded on each smaller sector $|\arg t| \leqq \pi / 2-$ $\omega-\varepsilon$, (Kato [10], Komatsu [12]).

THEOREm 10.1. If $A$ is of type $(\omega, M(\theta))$ and if $|\arg c|<\pi-\omega$, then $c A$ satisfies (1.5) and we have

$$
(c A)_{*}^{\alpha}=c^{\alpha} A_{*}^{\alpha} \quad \text { for all } \alpha .
$$

Proof. Since $(\lambda+c A)^{-1}=c^{-1}\left(c^{-1} \lambda+A\right)^{-1}$, the former statement is evident. To prove (10.2) it is enough to show that $(c A)_{*}^{\alpha} x=c^{\alpha} A_{*}^{\alpha} x$ holds for all $x \in D^{\sigma}$ with $\sigma$ sufficiently large (or $D^{\sigma} \cap R^{\sigma}$ or $R^{o}$ ). This is proved by rotating the integration path in (4.1) (or (4.11) or (4.10)) since the remainders $R_{+n}(\lambda, x)$ are estimated uniformly on each smaller sector.

Proposition 10.2. If $A$ is of type $(\omega, M(\theta))$ and if $0<\alpha<\pi / \omega$, then every $\lambda>0$ belongs to the resolvent set of $-A_{+}^{\alpha}$ and the resolvent is expressed by

$$
\begin{aligned}
\left(\lambda+A_{+}^{\alpha}\right)^{-1}= & \sum_{i=1}^{h}\left(\alpha \zeta_{i}^{\alpha-1}\right)^{-1}\left(-\zeta_{i}+A\right)^{-1} \\
& +\frac{\sin \pi \alpha}{\pi} \int_{0}^{\infty} \frac{\tau^{\alpha}}{\lambda^{2}+2 \lambda \tau^{\alpha} \cos \pi \alpha+\tau^{2 \alpha}}(\tau+A)^{-1} d \tau,
\end{aligned}
$$

where $\zeta_{i}$ 's are all roots of $\lambda+\zeta^{\alpha}=0$ satisfying $-\pi<\arg \zeta \leqq \pi$, and the integral term disappears when $\alpha$ is an integer.

REMARK. The number $h$ of $\zeta_{i}$ is 0 when $0<\alpha<1$, 1 when $\alpha=1$, 2 when $1<\alpha<3$, 3 when $\alpha=3$, etc. This formula was used by Kato [10] to define $A^{\alpha}$ for $0<\alpha<1$. A different proof is given by Balakrishnan [2] for sufficiently small complex $\alpha$.

Proof. Let us denote the right-hand side of (10.3) by $I(\lambda, \alpha)$. It is clear that the integral converges in the uniform operator topology, and hence $I(\lambda, \alpha)$ is a bounded linear operator. If we prove that 


$$
\left(\lambda+A_{+}^{\alpha}\right) I(\lambda, \alpha) x=I(\lambda, \alpha)\left(\lambda+A_{+}^{\alpha}\right) x=x
$$

holds for every $x \in D^{\sigma}$ with sufficiently large $\sigma$, then the proposition follows easily as the limits of both sides.

Since the integrand in (10.3) commutes with $A$ and $(\lambda+A)^{-1}$, the commutativity is easy to see. Now we note that

$$
\begin{aligned}
I(\lambda, \alpha)= & \frac{\sin \pi \alpha}{\pi} \int_{0}^{\varepsilon} \frac{\tau^{\alpha}}{\lambda^{2}+2 \lambda \tau^{\alpha} \cos \pi \alpha+\tau^{2 \alpha}}(\tau+A)^{-1} d \tau \\
& -\frac{1}{2 \pi i} \int_{\Gamma_{\varepsilon}}\left(\lambda+\zeta^{\alpha}\right)^{-1}(-\zeta+A)^{-1} d \zeta,
\end{aligned}
$$

where $\varepsilon$ is a small positive number and the integration path $\Gamma_{\varepsilon}$ runs from $\infty e^{i(\omega+\Rightarrow)}$ to $-\varepsilon+i 0$ and from $-\varepsilon-i 0$ to $\infty e^{-i(\omega+\varepsilon)}$. This is proved by the standard technique of shift of integration path. Hence it follows that $I(\lambda, \alpha)$ is analytic in $\alpha \in(0, \pi / \omega)$. If $x \in D^{\sigma},\left(\lambda+A_{+}^{\alpha}\right) x$ is analytic in $\alpha \in(0, \sigma)$, so that $I(\lambda, \alpha)\left(\lambda+A_{+}^{\alpha}\right) x$ is an analytic function of $\alpha$ in the interval $0<\alpha<\min (\pi / \omega, \sigma)$. Thus it is sufficient to prove (10.4) only for $0<\alpha<1$. Let $x \in D(A)$. Then in a similar way to Proposition 4.9 we obtain

$$
\begin{aligned}
I(\lambda, \alpha) A_{+}^{\alpha} x= & \frac{\sin \pi \alpha}{\pi}\left[\int_{0}^{\infty} \frac{\tau^{\alpha-1}}{\lambda+(-\tau+i 0)^{\alpha}}(\tau+A)^{-1} A x d \tau\right. \\
& \left.+\int_{0}^{\infty} \frac{\tau^{\alpha}(-\tau-i 0)^{\alpha-1}}{\lambda^{2}+2 \lambda \tau^{2} \cos \pi \alpha+\tau^{2 \alpha}}(\tau+A)^{-1} A x d \tau\right] \\
= & \frac{\sin \pi \alpha}{\pi} \int_{0}^{\infty} \frac{\lambda \tau^{\alpha-1}}{\lambda^{2}+2 \lambda \tau^{\alpha} \cos \pi \alpha+\tau^{2 \alpha}}\left(1-\tau(\tau+A)^{-1}\right) x d \tau .
\end{aligned}
$$

Now it is easy to see that this is equal to $x-\lambda I(\lambda, \alpha) x$.

Theorem 10.3 (Kato [10]). If $A$ is of type $(\omega, M(\theta)$ ) and if $0<\alpha<\pi / \omega$, then $A^{\alpha}$ is of type $\left(\alpha \omega, M_{\alpha}(\theta)\right)$, where

$$
M_{\alpha}(\theta) \leqq\left(\frac{h}{\alpha}+\left|1-\frac{h}{\alpha}\right|\right) M\left(\left(\frac{1}{\alpha} \theta+\frac{\alpha-1}{\alpha} \pi\right)^{+}\right),
$$

with $h$ as in Proposition 10.2, and the resolvent $\left(\lambda+A^{\alpha}\right)^{-1}$ is analytic in $\alpha$ and $\lambda$ in the domain $0<\alpha<\pi / \omega,|\arg \lambda|<\pi-\alpha \omega$.

Proof. When $\arg \theta=0$, this is an easy consequence of the representation (10.3), since we have

$$
\frac{\sin \pi \alpha}{\pi} \int_{0}^{\infty} \frac{\tau^{\alpha-1}}{\lambda^{2}+2 \lambda \tau^{\alpha} \cos \pi \alpha+\tau^{2 \alpha}} d \tau=\left(1-\frac{h}{\alpha}\right) \frac{1}{\lambda} .
$$

The case $\arg \theta \neq 0$ can be reduced to this case because of Theorem 10.1. The analyticity follows from (10.5). 
Proposition 10.4. If $x \in \overline{R(A)}$, then $\left(\lambda+A_{+}^{\alpha}\right)^{-1} x$ converges strongly to $(\lambda+1)^{-1} x$ as $\alpha>0$ tends to 0 .

Proof. We may consider only the case $\lambda>0$. Since $\left(\lambda+A_{+}^{\alpha}\right)^{-1}$ is bounded uniformly in $\alpha$ and $D(A) \cap R(A)$ is dense in $\overline{R(A)}$, it is enough to show that

$$
\left(\lambda+A^{\alpha}\right)^{-1} x \rightarrow(\lambda+1)^{-1} x, \quad x \in D(A) \cap R(A) .
$$

We have

$$
\begin{aligned}
(\lambda & \left.+A^{\alpha}\right)^{-1} x-(\lambda+1)^{-1} x \\
& =\frac{\sin \pi \alpha}{\pi} \int_{0}^{\infty} \frac{\tau^{\alpha}}{\lambda^{2}+2 \lambda \tau^{\alpha} \cos \pi \alpha+\tau^{2 \alpha}}\left((\tau+A)^{-1}-(\tau+1)^{-1}\right) x d \tau .
\end{aligned}
$$

If $\alpha$ is sufficiently small, $\tau^{\alpha}\left(\lambda^{2}+2 \lambda \tau^{\alpha} \cos \pi \alpha+\tau^{2 \alpha}\right)^{-1}$ is uniformly bounded. Further, from the assumption that $x \in D(A) \cap R(A)$ it follows that

$$
\left\|\left((\tau+A)^{-1}-(\tau+1)^{-1}\right) x\right\|=\left\|(\tau+1)^{-1}(\tau+A)^{-1}(1-A) x\right\| \leqq C(\tau+1)^{-2} .
$$

Thus we have

$$
\left\|\left(\lambda+A^{\alpha}\right)^{-1} x-(\tau+1)^{-1} x\right\| \leqq \frac{\sin \pi \alpha}{\pi} \int_{0}^{\infty} \frac{C_{1}}{(\tau+1)^{2}} d \tau \rightarrow 0 .
$$

Theorem 10.5 (Yosida [22], Kato [10], Watanabe [20]). If $A$ is an operator of type $(\omega, M(\theta))$ and $0<\alpha \omega<\pi / 2$, then $-A_{+}^{\alpha}$ is the generator of strongly continuous semi-group $\exp \left(-t A_{+}^{\alpha}\right)$ which is analytic in the sector $|\arg t|<\pi / 2-\alpha \omega$ and uniformly bounded on each smaller sector $|\arg t| \leqq \pi / 2-\alpha \omega-\varepsilon, \varepsilon>0$. $\quad \exp \left(-t A_{+}^{\alpha}\right)$ is continuous in $t$ and $\alpha$ in the uniform operator topology in the domain $|\arg t|<\pi / 2-\alpha \omega, \quad 0<\alpha<\pi /(2 \omega)$. Moreover, if $x \in \overline{R(A)}$, then $\exp \left(-t A_{+}^{\alpha}\right) x$ converges strongly to $\exp (-t) x$ uniformly on every compact set of the half plane $\operatorname{Re} t>0$ as $\alpha>0$ tends to 0 .

Proof. The first and the second statements are direct consequences of Theorem 10.3. The third statement follows from Proposition 10.4 and Theorem 3.2 by Trotter's theorem (Trotter [19]).

Theorem 10.6 (Watanabe [20]). Let $A$ be of type $(\omega, M(\theta))$. Then

$$
\left(A_{+}^{\alpha}\right)_{+}^{\beta}=A_{+}^{\alpha \beta}
$$

if $0<\alpha<\pi / \omega$ and $\operatorname{Re} \beta>0$.

Proof. $\left(A_{+}^{\alpha}\right)_{+}^{\beta}$ and $A_{+}^{\alpha \beta}$ are defined to be the smallest closed exten- 
sions of their restrictions to $D^{\sigma}\left(A_{+}^{\alpha}\right)$ and $D^{\tau}(A)$, respectively, where $\sigma$ and $\tau$ are sufficiently large. On the other hand, since $\left(A_{+}^{\alpha}\right)^{n}=A_{+}^{\alpha n}$ for positive integer $n$ by Theorem 7.1, it follows from Theorem 6.5 that $D^{\sigma}\left(A_{+}^{\alpha}\right)=D\left(\left(A_{+}^{\alpha}\right)^{\sigma}\right)=D\left(A_{+}^{\alpha \sigma}\right) \subset D^{\alpha \sigma-\varepsilon}(A) \subset D\left(A_{+}^{\alpha(\sigma-1)}\right)=D^{\sigma-1}\left(A_{+}^{\alpha}\right)$ for positive integer $\sigma$. Thus we have only to prove that

$$
\left(A_{+}^{\alpha}\right)_{+}^{\beta} x=A_{+}^{\alpha \beta} x, \quad x \in D^{\tau}(A)
$$

holds if $\tau$ is sufficiently large. We may assume that $\tau=\alpha(\sigma-1 / 2)$ with a positive integer $\sigma$. Then, both sides of (10.8) are analytic in $\beta$ in the domain $0<\operatorname{Re} \beta<\sigma-1$. Thus it is enough to show (10.8) for $0<\beta<\min \{1,1 / \alpha\}$, and, say, for $x \in D(A)$.

By an easy computation it follows from (10.5) that

$$
A_{+}^{\alpha}\left(\lambda+A_{+}^{\alpha}\right)^{-1}=-\frac{1}{2 \pi i} \int_{\Gamma_{\theta}} \zeta^{\alpha}\left(\lambda+\zeta^{\alpha}\right)^{-1} \zeta^{-1}(-\zeta+A)^{-1} A x d \zeta
$$

for $\lambda>0$ and $x \in D(A)$, where $\Gamma_{\theta}$ is composed of two rays $r e^{i \theta}$, $\infty>r \geqq 0$, and $r e^{-i \theta}, 0 \leqq r<\infty$, and $\theta$ must satisfy $\pi>\alpha \theta>\alpha \omega$.

Therefore we have

$$
\begin{aligned}
\left(A_{+}^{\alpha}\right)_{+}^{\beta} x & =\frac{\sin \pi \beta}{\pi} \int_{0}^{\infty} \lambda^{\beta-1} A_{+}^{\alpha}\left(\lambda+A_{+}^{\alpha}\right)^{-1} x d \lambda \\
& =-\frac{1}{2 \pi i} \int_{\Gamma_{\theta}} \zeta^{-1}(-\zeta+A)^{-1} A x d \zeta \frac{\sin \pi \beta}{\pi} \int_{0}^{\infty} \lambda^{\beta-1} \zeta^{\alpha}\left(\lambda+\zeta^{\alpha}\right)^{-1} d \lambda \\
& =-\frac{1}{2 \pi i} \int_{\Gamma_{\theta}} \zeta^{\alpha \beta-1}(-\zeta+A)^{-1} A x d \zeta, \quad x \in D(A) .
\end{aligned}
$$

We may let $\theta=\pi$ in the last integral without changing the value and obtain

$$
\left(A_{+}^{\alpha}\right)_{+}^{\beta} x=\frac{\sin \pi \alpha \beta}{\pi} \int_{0}^{\infty} \tau^{\alpha \beta-1}(\tau+A)^{-1} A x d \tau=A_{+}^{\alpha \beta} x .
$$

11. The case where $-A$ generates a bounded semi-group. Throughout this section we assume that $-A$ is the infinitesimal generator of a strongly continuous semi-group $T_{t}$ which is uniformly bounded in $t$ :

$$
\left\|T_{t}\right\| \leqq M, \quad 0 \leqq t<\infty .
$$

A closed linear operator $A$ satisfies this condition if and only if the domain $D(A)$ is dense, the negative real axis is contained in the resolvent set $\rho(A)$ and

$$
\left\|\lambda^{m}(\lambda+A)^{-m}\right\| \leqq M, \quad 0<\lambda<\infty, \quad m=1,2, \cdots .
$$

A typical example is the differential operator $p=-d / d s$ in the 
space $B[0, \infty)$ of all bounded uniformly continuous functions $x(s)$ on $[0, \infty)$. The corresponding semi-group $T_{t}$ is given by

$$
T_{t} x(s)=x(s+t), \quad x(s) \in B[0, \infty) .
$$

It is well-known that the half-plane $\operatorname{Re} \lambda>0$ is contained in the resolvent set $\rho(-A)$ and the resolvent is expressible as the Laplace transform of the semi-group $T_{t}$ :

$$
(\lambda+A)^{-1} x=\int_{0}^{\infty} e^{-\lambda t} T_{t} x d t, \quad \operatorname{Re} \lambda>0 .
$$

Thus $T_{t}$ is commutative with $(\lambda+A)^{-1}$. We note that hence it follows that $T_{t} A^{n} \subset A^{n} T_{t}, n=1,2, \cdots, T_{t} A_{+}^{\alpha} \subset A_{+}^{\alpha} T_{t}, \operatorname{Re} \alpha>0$, and $T_{t} A_{0}^{\alpha} \subset A_{0}^{\alpha} T_{t}$, $\alpha$ arbitrary. $A_{-}^{\alpha}, \operatorname{Re} \alpha<0$, coincides with $A_{0}^{\alpha}$ since $D(A)$ is dense. (11.4) implies also that $A$ is of type $(\pi / 2, M \sec \theta)$.

Proposition 11.1. Let $\operatorname{Re} \alpha<0$ and $\mu>0$. Then

$$
(\mu+A)_{0}^{\alpha} x=\frac{1}{\Gamma(-\alpha)} \int_{0}^{\infty} t^{-\alpha-1} e^{-\mu t} T_{t} x d t, \quad x \in X .
$$

Proof. If $0<-\alpha<1$, then we have by Proposition 5.1 and (11.4)

$$
\begin{aligned}
(\mu+A)_{0}^{\alpha} x & =\frac{1}{\Gamma(-\alpha) \Gamma(1+\alpha)} \int_{\mu}^{\infty}(\lambda-\mu)^{\alpha} d \lambda \int_{0}^{\infty} e^{-\lambda t} T_{t} x d t \\
& =\frac{1}{\Gamma(-\alpha)} \int_{0}^{\infty} t^{-\alpha-1} e^{-\mu t} T_{t} x d t .
\end{aligned}
$$

Since both sides of (11.5) are analytic in $\alpha$, the equality holds for all $\operatorname{Re} \alpha<0$.

Definition. Let $\sigma=n+\rho \geqq 0$ as before. $C^{\sigma}=C^{\sigma}(A)$ is defined to be the set of $x \in X$ such that

(i) $x \in D\left(A^{n}\right)$, and, in case $\rho>0$,

(ii) $T_{t} A^{n} x-A^{n} x=O\left(t^{\rho}\right)$ as $t \rightarrow 0$.

Let

$$
p_{o}(x)= \begin{cases}\left\|A^{n} x\right\| & \rho=0 \\ \sup _{t} t^{-\rho}\left\|T_{t} A^{n} x-A^{n} x\right\| & \rho>0\end{cases}
$$

Then $C^{\sigma}$ becomes a normed space by the norm

$$
\|x\|+p_{\sigma}(x) \text {. }
$$

When $A=-d / d s$ in $B[0, \infty), C^{\sigma}$ is the set of all $n$-times con- 
tinuously differentiable functions $x(s)$ on $[0, \infty)$ such that the $n$-th derivative $x^{(n)}(s)$ is uniformly Hölder continuous with exponent $\rho$ or simply uniformly continuous when $\rho=0$.

Proposition 11.2. $C^{\sigma}$ is a Banach space and contained in $D^{\sigma}$ continuously.

Proof. If $\sigma=n$, then $C^{o}=D^{o}$ and these spaces are isomorphic. As we have seen in the proof of Proposition 4.2, the operator $A^{n}$ is closed. Thus $C^{n}=D\left(A^{n}\right)$ is complete.

Next let $\rho>0$. We have

$$
q_{\sigma}(x) \leqq K(\sigma) p_{\sigma}(x), \quad x \in C^{\sigma},
$$

with a constant $K(\sigma)$ depending only on $\sigma$. In fact, it follows from (11.4) that

$$
\begin{aligned}
\left\|A^{n+1}(\lambda+A)^{-1} x\right\| & =\left\|\left(1-\lambda(\lambda+A)^{-1}\right) A^{n} x\right\| \\
& \leqq \int_{0}^{\infty} \lambda e^{-\lambda t}\left\|T_{t} A^{n} x-A^{n} x\right\| d t \\
& \leqq \Gamma(1+\rho) \lambda^{-\rho} p_{\sigma}(x) .
\end{aligned}
$$

Hence we have $C^{\sigma} \subset D^{\sigma}$. (11.7) implies also that the embedding is continuous.

To prove the completeness of $C^{\sigma}$, let $x_{j} \in C^{\sigma}$ be a Cauchy sequence. By (11.7) and Proposition 4.7, $x_{j}$ forms a Cauchy sequence in $C^{n}$. Thus there is an $x \in C^{n}$ such that $x_{j} \rightarrow x$ and $A^{n} x_{j} \rightarrow A^{n} x$ in $X$. It is easy to see that

$$
p_{o}\left(x_{j}-x\right)=\sup _{t} \lim _{k \rightarrow \infty} t^{-\rho}\left\|\left(T_{t}-1\right) A^{n}\left(x_{j}-x_{k}\right)\right\| \rightarrow 0 .
$$

TheOREM 11.3. Let $\operatorname{Re} \alpha>0$. If $\alpha$ is real or $\operatorname{Re} \alpha$ is not an integer, then $D\left(A_{+}^{\alpha}\right)$ is contained in $C^{\mathrm{Re} \alpha}$.

Proof. Since $D(A)$ is dense, $D\left(A_{+}^{n}\right)$ coincides with $D\left(A^{n}\right)=C^{n}$. Suppose that $\operatorname{Re} \alpha$ is not an integer. By Theorem 7.1, $x \in D\left(A_{+}^{\alpha}\right)$ if and only if $x \in D\left(A^{n}\right)$ and $A^{n} x \in D\left(A_{+}^{\alpha-n}\right)$. On the other hand, $x \in C^{\sigma}$ if and only if $x \in D\left(A^{n}\right)$ and $A^{n} x \in C^{\sigma-n}$. Therefore we may assume that $0<\operatorname{Re} \alpha<1$ without loss of generality. If $x \in D\left(A_{+}^{\alpha}\right)=D\left((\mu+A)_{+}^{\alpha}\right)$, $\mu>0$, then there is a $y \in X$ such that $x=(\mu+A)_{0}^{-\alpha} y$. Thus we have

$$
\begin{aligned}
T_{h} x-x= & \frac{1}{\Gamma(\alpha)}\left[\int_{h}^{\infty}\left\{(t-h)^{\alpha-1} e^{-\mu(t-h)}-t^{\alpha-1} e^{-\mu t}\right\} T_{t} y d t\right. \\
& \left.-\int_{0}^{h} t^{\alpha-1} e^{-\mu t} T_{t} y d t\right] .
\end{aligned}
$$


Clearly the norm of the latter integral is bounded by $M \sigma^{-1} h^{\sigma}\|y\|$ with $\sigma=\operatorname{Re} \alpha$. The former integral is estimated as follows:

$$
\begin{aligned}
M & \|y\| \int_{h}^{\infty}\left|(t-h)^{\alpha-1} e^{-\mu(t-h)}-t^{\alpha-1} e^{-\mu t}\right| d t \\
& \leqq M\|y\| \frac{|1-\alpha|}{1-\sigma} \int_{h}^{\infty} d t \int_{t-h}^{t}\left(\frac{1-\sigma}{s}+\mu\right) s^{\sigma-1} e^{-\mu s} d s \\
& \leqq \frac{|1-\sigma|}{\sigma(1-\sigma)} M h^{\sigma}\|y\| .
\end{aligned}
$$

Proposition 11.4 (Balakrishnan [1]). Let $\sigma>\operatorname{Re} \alpha>0$. Then for every $x \in D^{\sigma}$

$$
A_{+}^{\alpha} x=\frac{1}{\Gamma(-\alpha)} \int_{0}^{\infty} t^{-\alpha-1} T_{t} x d t
$$

in the sense of Sato or

$$
\begin{aligned}
A_{+}^{\alpha} x= & \frac{1}{\Gamma(-\alpha)}\left[\int_{0}^{N} t^{-\alpha-1}\left(T_{t} x-x-t A x-\cdots-\frac{t^{n}}{n !} A^{n} x\right) d t\right. \\
& \left.+\sum_{k=0}^{n} \frac{N^{k-\alpha}}{k-\alpha} A^{k} x+\int_{N}^{\infty} t^{-\alpha-1} T_{t} x d t\right],
\end{aligned}
$$

where $0<N<\infty$. In particular, if $0<\operatorname{Re} \alpha<1$ and $\sigma>\operatorname{Re} \alpha$, then

$$
A_{+}^{\alpha} x=\frac{1}{\Gamma(-\alpha)} \int_{0}^{\infty} t^{-\alpha-1}\left(T_{t} x-x\right) d t, \quad x \in D^{\sigma} .
$$

Proof. If $x \in D^{\sigma}$, it follows from Theorem 11.3 that $x \in C^{\sigma-\varepsilon}$ for $\varepsilon>0$. Thus the integrals of (11.8)' converge absolutely. Further, it is easy to see that the right-hand side of (11.8)' represents an analytic function of $\alpha$ for $0<\operatorname{Re} \alpha<\sigma$ independent of $N$. In case $0<\operatorname{Re} \alpha<$ $\sigma<1$, we can make $N$ tend to infinity and obtain (11.9). Since $A_{+}^{\alpha} x$ is also analytic in $\alpha$ and $(11.8)^{\prime}$ does not depend on $\sigma>\operatorname{Re} \alpha$, it is enough to prove equality (11.9). By (4.2) and (11.4) we have

$$
\begin{aligned}
A_{+}^{\alpha} x & =-\frac{\sin \pi \alpha}{\pi} \int_{0}^{\infty} \lambda^{\alpha} d \lambda \int_{0}^{\infty} e^{-\lambda t}\left(T_{t} x-x\right) d t \\
& =\frac{1}{\Gamma(-\alpha)} \int_{0}^{\infty} t^{-\alpha-1}\left(T_{t} x-x\right) d t,
\end{aligned}
$$

completing the proof.

TheOREM 11.5. Let $p=-d / d s$ in $B[0, \infty)$ and $\operatorname{Re} \alpha>0$. Then $x \in D\left(A_{+}^{\alpha}\right)$ if and only if 


$$
\left\langle T_{8} x, y^{\prime}\right\rangle \in D\left(p_{+}^{\alpha}\right) \text { for all } y^{\prime} \in X^{\prime} \text {, and }
$$

there is a function $f(s) \in X$ independent of $y^{\prime}$ such that

$$
p_{+}^{\alpha}\left\langle T_{s} x, y^{\prime}\right\rangle=\left\langle f(s), y^{\prime}\right\rangle .
$$

If $X$ is sequentially weakly complete, the second condition may be omitted.

Proof. First we remark that $\left\langle T_{s} y, y^{\prime}\right\rangle \in B[0, \infty)$ for all $y \in X$ and $y^{\prime} \in X^{\prime}$. In view of Proposition 11.1 we have

$$
\begin{aligned}
\left\langle T_{s}(\mu+A)_{0}^{-\alpha} y, y^{\prime}\right\rangle & =\frac{1}{\Gamma(\alpha)} \int_{s}^{\infty}(t-s)^{\alpha-1} e^{-\mu(t-s)}\left\langle T_{t} y, y^{\prime}\right\rangle d t \\
& =(\mu+p)_{0}^{-\alpha}\left\langle T_{s} y, y^{\prime}\right\rangle
\end{aligned}
$$

for $\mu>0$. Let $x \in D\left(A_{+}^{\alpha}\right)$. Then there is a $y \in X$ such that $x=(\mu+A)_{0}^{-\alpha} y$. Thus we have by (11.12)

$$
\left\langle T_{s} x, y^{\prime}\right\rangle=(\mu+p)_{0}^{-\alpha}\left\langle T_{s} y, y^{\prime}\right\rangle \in D\left(p_{+}^{\alpha}\right) .
$$

Similarly it is shown by Propositions 6.2 and 6.3 that

$$
p_{+}^{\alpha}\left\langle T_{s} x, y^{\prime}\right\rangle=\left\langle T_{s} A_{+}^{\alpha} x, y^{\prime}\right\rangle, \quad x \in D\left(A_{+}^{\alpha}\right) .
$$

This proves (11.11) with $f(s)=T_{s} A_{+}^{\alpha} x$.

To prove the converse, first let $\alpha$ be an integer $n$. Since assumptions (11.10) and (11.11) show that $T_{s} x$ is $n$ times weakly differentiable at $s=0$, it follows that $x \in D(A)$ (see Komatsu [12]), and we have by (11.13)

$$
p\left\langle T_{s} x, y^{\prime}\right\rangle=\left\langle T_{s} A x, y^{\prime}\right\rangle .
$$

Assume that the theorem is true for smaller integers. Then it follows from (11.14) that $A x \in D\left(A^{n-1}\right)$. Hence we have $x \in D\left(A^{n}\right)$.

Next if $\alpha$ is not an integer, take an integer $n$ such that $n+1>\operatorname{Re} \alpha$. (11.12) with Theorem 7.1 gives

$$
\left\langle T_{s}(\mu+A)_{0}^{-\beta} x, y^{\prime}\right\rangle=(\mu+p)_{0}^{-\beta}\left\langle T_{s} x, y^{\prime}\right\rangle \in D\left((\mu+p)^{n+1}\right)=D\left(p^{n+1}\right),
$$

if $\beta=n+1-\alpha$ and $\mu>0$. Moreover, we have by Propositions 6.2 and 6.3

$$
\begin{aligned}
p^{n+1} & \left\langle T_{s}(\mu+A)_{0}^{-\beta} x, y^{\prime}\right\rangle \\
& =p_{+}^{\beta}(\mu+p)_{0}^{-\beta} p_{+}^{\alpha}\left\langle T_{s} x, y^{\prime}\right\rangle \\
& =\left(p(\mu+p)^{-1}\right)_{+}^{\beta}\left\langle f(s), y^{\prime}\right\rangle \\
& =\left\langle f(s), y^{\prime}\right\rangle-\frac{\sin \pi \beta}{\pi} \int_{0}^{\mu} \lambda^{\beta}(\mu-\lambda)^{-\beta} d \lambda \int_{s}^{\infty} e^{-\lambda(t-s)}\left\langle f(t), y^{\prime}\right\rangle d t .
\end{aligned}
$$


Since $f(s)$ is uniformly bounded and weakly continuous, it can be shown that the integral $\int_{0}^{\mu} \lambda^{\beta}(\mu-\lambda)^{-\beta} d \lambda \int_{s}^{\infty} e^{-\lambda(t-s)} f(t) d t$ converges weakly (see Komatsu [12]). Thus there exists a function $g(s) \in X$ such that

$$
p^{n+1}\left\langle T_{s}(\mu+A)_{0}^{-\beta} x, y^{\prime}\right\rangle=\left\langle g(s), y^{\prime}\right\rangle, \quad y^{\prime} \in X^{\prime} .
$$

Applying the theorem in the case of integer $n+1$, we obtain $(\mu+A)_{0}^{-\beta} x \in D\left(A^{n+1}\right)$. Since $D(A)$ is dense, it follows from Theorem 7.1 that $x \in D\left((\mu+A)_{+}^{\alpha}\right)=D\left(A_{+}^{\alpha}\right)$.

Lastly let us prove that if $X$ is sequentially weakly complete, the existence of $f(s) \in X$ satisfying (11.11) follows from (11.10). Let $x$ satisfy (11.10). In view of Theorem 11.3 we have $\left\langle T_{s} x, y^{\prime}\right\rangle \in C^{\sigma}(p)$ for any $\sigma<\operatorname{Re} \alpha$, i.e., $x(s)=T_{s} x$ is $n$ times scalarly differentiable and the $n$-th derivative is scalarly uniformly Hölder continuous with exponent $\rho, \sigma=n+\rho$.

If $\rho>0$, we can replace the word "scalarly" by "strongly". It is evident that $x(s)$ is $n$ times weakly* continuously differentiable in $X^{\prime \prime}$ and $\left\{\left(x^{(n)}(s)-x^{(n)}(t)\right) /|t-s|^{\rho}\right\}$ is weakly* bounded in $X^{\prime \prime}$. Since a weakly* bounded set is strongly bounded, $x^{(n)}(s)$ is strongly uniformly Hölder continuous in $X^{\prime \prime}$. Hence it is easily proved that $x^{(k)}(s)$, $k=1,2, \cdots, n$, is strongly continuous in $X^{\prime \prime}$. In particular the difference quotient

$$
\frac{x(s+h)-x(s)}{h}=\frac{1}{h} \int_{s}^{s+h} x^{\prime}(t) d t
$$

converges strongly to $x^{\prime}(s)$ in $X^{\prime \prime}$ as $h \rightarrow 0$. This proves that $x(s)$ is strongly differentiable. Repeating the same argument, we see that $x(s)$ is $n$ times strongly differentiable and the $n$-th derivative is strongly uniformly Hölder continuous with exponent $\rho$.

As we have shown above, it then follows that $x \in D\left(A^{n}\right)$ and $(-d / d s)^{n} T_{s} x=T_{s} A^{n} x$. Therefore $x$ belongs to $C^{o}(A)$. In particular we have $x \in D\left(A_{+}^{\beta}\right)$ for $\operatorname{Re} \beta<\operatorname{Re} \alpha$ and

$$
p_{+}^{\beta}\left\langle T_{s} x, y^{\prime}\right\rangle=\left\langle T_{s} A_{+}^{\beta} x, y^{\prime}\right\rangle, \quad y^{\prime} \in X^{\prime} .
$$

When $\beta$ tends to $\alpha$ in a Stolz region, the left-hand side converges to $p_{+}^{\alpha}\left\langle T_{s} x, y^{\prime}\right\rangle$. Since $X$ is sequentially weakly complete, there exists the limit

$$
f(s)=\mathrm{w}-\lim _{\beta \rightarrow \alpha} T_{s} A_{+}^{\beta} x \in X,
$$

which satisfies (11.11).

Incidentally, we have proved that if $x$ satisfies (11.10), then $x \in D\left(A_{+}^{\beta}\right)$ for $\operatorname{Re} \beta<\operatorname{Re} \alpha$ in any case.

Similarly to Theorem 7.6 the following theorem holds. 
Theorem 11.6 (Hardy-Littlewood [6]). (i) Let $\sigma>\operatorname{Re} \alpha>0$. If $\sigma-\operatorname{Re} \alpha$ is not an integer, then $A_{+}^{\alpha}\left(C^{\sigma}\right)$ is contained in $C^{\sigma-\operatorname{Re} \alpha}$. (ii) If $\operatorname{Re} \alpha<0$ and if $\sigma-\operatorname{Re} \alpha$ is not an integer, then $A_{0}^{\alpha}\left(C^{\sigma} \cap D\left(A_{0}^{\alpha}\right)\right)$ is contained in $C^{o-\operatorname{Re} \alpha}$.

Proof. (i) We may assume that $0<\operatorname{Re} \alpha<1$ without loss of generality. First let $\operatorname{Re} \alpha<\rho$. (11.9) gives

$$
\left(T_{h}-1\right) A^{n} A_{+}^{\alpha} x=\frac{1}{\Gamma(-\alpha)} \int_{0}^{\infty} t^{-\alpha-1}\left(T_{h}-1\right)\left(T_{t}-1\right) A^{n} x d t .
$$

Hence, using the estimate

$$
\left\|\left(T_{h}-1\right)\left(T_{t}-1\right) A^{n} x\right\| \leqq(M+1) p_{o}(x) \min \left\{h^{\rho}, t^{\rho}\right\},
$$

we obtain

$$
\left\|\left(T_{h}-1\right) A^{n} A_{+}^{\alpha} x\right\| \leqq \frac{(M+1) p_{o}(x)}{|\Gamma(-\alpha)|}\left(\frac{1}{\rho-\operatorname{Re} \alpha}+\frac{1}{\operatorname{Re} \alpha}\right) h^{\rho-\operatorname{Re} \alpha} .
$$

In case $\rho<\operatorname{Re} \alpha$, we employ the identity

$$
\begin{aligned}
\left(T_{h}-1\right) A^{n-1} A_{+}^{\alpha} x= & \frac{1}{\Gamma(-\alpha)}\left[\int_{0}^{h} t^{-\alpha-1} d t \int_{0}^{t} T_{s}\left(T_{h}-1\right) A^{n} x d s\right. \\
& \left.+\int_{h}^{\infty} t^{-\alpha-1} d t \int_{0}^{h} T_{s}\left(T_{t}-1\right) A^{n} x d s\right],
\end{aligned}
$$

which follows from (11.9) and Theorem 11.5. From the estimate

$$
\left\|\int_{0}^{t} T_{s}\left(T_{h}-1\right) A^{n} x d s\right\| \leqq M p_{\sigma}(x) t h^{\rho}
$$

and the one with $t$ and $h$ interchanged, it is concluded that

$$
\left(T_{h}-1\right) A^{n-1} A_{+}^{\alpha} x=O\left(h^{\rho-\operatorname{Re} \alpha+1}\right), \quad \text { as } \quad h \rightarrow 0 .
$$

(ii) The proof of (ii) in Theorem 7.6 can be modified to this case with no difficulty.

Theorem 11.7. If $0<\alpha<1, \lambda>0$ and $n=1,2, \cdots$, then there is an analytic function $\varphi^{n}(\alpha, \lambda, s)>0$ defined on $(0, \infty)$, independent of $A$, such that

$$
\left(\lambda+A_{+}^{\alpha}\right)^{-n} x=\lambda^{-n} \int_{0}^{\infty} \varphi^{n}(\alpha, \lambda, s) T_{s} x d s .
$$

$\varphi^{n}(\alpha, \lambda, s)$ satisfies

$$
\int_{0}^{\infty} \varphi^{n}(\alpha, \lambda, s) d s=1
$$


In particular we have

$$
\left\|\left(\lambda\left(\lambda+A_{+}^{\alpha}\right)^{-1}\right)^{n}\right\| \leqq M, \quad n=1,2, \cdots .
$$

Proof. Combining (11.4) and (10.3), we have

$$
\begin{aligned}
\left(\lambda+A_{+}^{\alpha}\right)^{-1} x & =\frac{\sin \pi \alpha}{\pi} \int_{0}^{\infty} \frac{\tau^{\alpha} d \tau}{\lambda^{2}+2 \lambda \tau^{\alpha} \cos \pi \alpha+\tau^{2 \alpha}} \int_{0}^{\infty} e^{-\tau s} T_{s} x d s \\
& =\lambda^{-1} \int_{0}^{\infty} \varphi^{1}(\alpha, \lambda, s) T_{s} x d s
\end{aligned}
$$

where

$$
\varphi^{1}(\alpha, \lambda, s)=\frac{\lambda \sin \pi \alpha}{\pi} \int_{0}^{\infty} \frac{\tau^{\alpha} e^{-\tau s}}{\lambda^{2}+2 \lambda \tau^{\alpha} \cos \pi \alpha+\tau^{2 \alpha}} d \tau
$$

It is easy to see that $\varphi^{1}(\alpha, \lambda, s)$ is positive and analytic for $0<s<\infty$ and satisfies (11.16). Let us define $\varphi^{n}(\alpha, \lambda, s)$ for $n>1$ recursively by

$$
\begin{aligned}
\varphi^{n+1}(\alpha, \lambda, s) & =\varphi^{n}(\alpha, \lambda, s) * \varphi^{1}(\alpha, \lambda, s) \\
& =\int_{0}^{s} \phi^{n}(\alpha, \lambda, s-t) \varphi^{1}(\alpha, \lambda, t) d t .
\end{aligned}
$$

(11.15) for general $n$ is proved by induction.

Other properties of $\varphi^{n}(\alpha, \lambda, s)$ are clear by the definition.

Theorem 11.8 (Phillips [16], Yosida [22]). If $0<\alpha<1$, then there exists a positive analytic function $\psi(\alpha, t, s)$ on $(0, \infty)^{2}$ such that

$$
\int_{0}^{\infty} \psi(\alpha, t, s) d s=1
$$

and the analytic semi-group $\exp \left(-t A_{+}^{\alpha}\right)$ generated by $-A_{+}^{\alpha}$ has the representation

$$
\exp \left(-t A_{+}^{\alpha}\right) x=\int_{0}^{\infty} \psi(\alpha, t, s) T_{s} x d s .
$$

$\exp \left(-t A_{+}^{\alpha}\right)$ is uniformly bounded:

$$
\left\|\exp \left(-t A_{+}^{\alpha}\right)\right\| \leqq M, 0<t<\infty, 0<\alpha<1,
$$

and converges strongly to $T_{t}=\exp (-t A)$ as $\alpha$ tends to 1 . The convergence is uniform on every compact set in $(0, \infty)$.

Proof. Let

$$
\Psi^{n}(\alpha, t, s)=\int_{0}^{s} \phi^{n}(\alpha, n / t, r) d r
$$


Then we have by (11.15)

$$
\left(1+\frac{t A_{+}^{\alpha}}{n}\right)^{-n} x=\int_{0}^{\infty} T_{s} x d \Psi^{n}(\alpha, t, s)
$$

and in particular

$$
\left(1+\frac{t a^{\alpha}}{n}\right)^{-n} x=\int_{0}^{\infty} e^{-a s} d \Psi^{n}(\alpha, t, s), \quad a>0 .
$$

Since $\left\{\Psi^{n}\right\}$ is a uniformly bounded sequence of increasing functions, there is a subsequence which converges to an increasing function $\Psi(\alpha, t, s)$ except for a countable set. Now it is easy to see that

$$
\begin{aligned}
\int_{0}^{\infty} e^{-a s} d \Psi(\alpha, t, s) & =\lim _{n^{\prime} \rightarrow \infty} \int_{0}^{\infty} e^{-a s} d \Psi^{n^{\prime}}(\alpha, t, s) \\
& =\exp \left(-t \alpha^{\alpha}\right), \quad a>0 .
\end{aligned}
$$

Letting $a \rightarrow 0$, we have also

$$
\int_{0}^{\infty} d \Psi(\alpha, t, s)=1
$$

Thus it follows that

$$
\int_{0}^{\infty} f(s) d \Psi(\alpha, t, s)=\lim _{n^{\prime} \rightarrow \infty} \int_{0}^{\infty} f(s) d \Psi^{n^{\prime}}(\alpha, t, s)
$$

for every bounded continuous function $f(s)$. In particular $\left(1+t A_{+}^{\alpha} / n^{\prime}\right)^{-n^{\prime}} x$ converges weakly to $\int_{0}^{\infty} T_{s} x d \Psi(\alpha, t, s)$. On the other hand, $\left(1+t A_{+}^{\alpha} / n\right)^{-n} x$ converges strongly to $\exp \left(-t A_{+}^{\alpha}\right) x$ (Hille-Phillips [7]), so that we have

$$
\exp \left(-t A_{+}^{\alpha}\right) x=\int_{0}^{\infty} T_{s} x d \Psi(\alpha, t, s) .
$$

(11.23) shows that $\exp \left(-t \lambda^{\alpha}\right)$ is the Laplace transform of $d \Psi(\alpha, t, s)$. It is not difficult to derive from this fact that $d \Psi(\alpha, t, s)$ has an analytic density $\psi(\alpha, t, s)$.

According to Trotter [19], a sequence of semi-groups $T_{t}^{\alpha}$ converges strongly to a semi-group $T_{t}$ if $T_{t}^{\alpha}$ is uniformly bounded and the resolvent converges at a point $\lambda>0$. This is surely the case with $T_{t}^{\alpha}=\exp \left(-t A^{\alpha}\right)$ by (11.21) and Theorem 10.3.

12. The case where $-A$ generates an analytic semi-group.

THEOREM 12.1. Let $A$ be an operator of type $(\omega, M(\theta))$ with $\omega<\pi / 2$, and let $T_{t}$ be the analytic semi-group generated by $-A$. If $|\arg t|<\pi / 2-\omega, t \neq 0$, then $T_{t} x \in D\left(A_{+}^{\alpha}\right)$ for any $x$ and $\operatorname{Re} \alpha>0$, 
and we have

$$
A_{+}^{\alpha} T_{t} x=\frac{1}{2 \pi i} \int_{\Gamma}(-\lambda)^{\alpha} e^{t \lambda}(\lambda+A)^{-1} x d \lambda
$$

where the path $\Gamma$ consists of two rays from $\infty e^{-i \theta}$ to 0 and from 0 to $\infty e^{i \theta}$ with $\pi / 2<\theta<\pi / 2+|\arg t|$. There is a constant $C$ depending only on $\alpha, \varepsilon>0$ and $A$ such that

$$
\left\|A_{+}^{\alpha} T_{t}\right\| \leqq C|t|^{-\mathrm{R} \alpha} \alpha,|\arg t| \leqq \pi / 2-\omega-\varepsilon .
$$

Proof. Since $T_{t} x$ is analytic in the sector $|\arg t|<\pi / 2-\omega$, $T_{t} x \in D\left(A_{+}^{\alpha}\right)$ for any $\operatorname{Re} \alpha>0$ and $A_{+}^{\alpha} T_{t} x$ is analytic in $\alpha$. The righthand side of (12.1) expresses also an analytic function of $\alpha$. Thus it is enough to prove (12.1) for $0<\alpha<1$. If $x \in D(A)$, we have the integral representation

$$
T_{t} x=\frac{1}{2 \pi i} \int_{\Gamma} e^{t \lambda}(\lambda+A)^{-1} x d \lambda
$$

(Hille-Phillips [6]), so that we obtain by (11.9)

$$
\begin{aligned}
A_{+}^{\alpha} T_{t} x & =\frac{1}{\Gamma(-\alpha)} \int_{0}^{\infty} s^{-\alpha-1} d s \frac{1}{2 \pi i} \int_{\Gamma}\left(e^{s \lambda}-1\right) e^{t \lambda}(\lambda+A)^{-1} x d \lambda \\
& =\frac{1}{2 \pi i} \int_{\Gamma} e^{t \lambda}(\lambda+A)^{-1} x d \lambda \frac{1}{\Gamma(-\alpha)} \int_{0}^{\infty} s^{-\alpha-1}\left(e^{s \lambda}-1\right) d s \\
& =\frac{1}{2 \pi i} \int_{\Gamma}(-\lambda)^{\alpha} e^{t \lambda}(\lambda+A)^{-1} x d \lambda .
\end{aligned}
$$

Since both sides of (12.1) represent bounded operators, (12.1) holds for every $x$. (12.2) follows easily from (12.1). Conversely,

THEOREM 12.2. Let $T_{t}$ be a bounded semi-group and $-A$ be its generator. If there is a complex number $\alpha$ with $\operatorname{Re} \alpha>0$ such that

$$
\left\|A_{+}^{\alpha} T_{t}\right\| \leqq C t^{-\operatorname{Re} \alpha}, \quad t>0,
$$

with a constant $C$, then $A$ is of type $(\omega, M(\theta))$ for an $\omega<\pi / 2$.

Proof. Since $A_{+}^{\alpha}$ commutes with $T_{t}, A_{+}^{\alpha} T_{t} x=T_{t / 2} A_{+}^{\alpha} T_{t / 2} x$ is still in $D\left(A_{+}^{\alpha}\right)$ and we have $\left(A_{+}^{\alpha}\right)^{2} T_{t} x=A_{+}^{2 \alpha} T_{t} x=\left(A_{+}^{\alpha} T_{t / 2}\right)^{2} x$. Similarly we have $T_{t} x \in D\left(A_{+}^{n \alpha}\right)$ and $A_{+}^{n \alpha} T_{t} x=\left(A_{+}^{\alpha} T_{t / n}\right)^{n} x$ for any integer $n>0$. Thus it follows from (12.4) that

$$
\left\|A_{+}^{n \alpha} T_{t}\right\| \leqq(C n)^{n \operatorname{Re} \alpha} t^{-n \operatorname{Re} \alpha}, \quad t>0 .
$$

Taking an $n$ such that $\operatorname{Re} n \alpha>1$ and applying Theorem 8.1, we obtain 
the estimate

$$
\left\|A T_{t}\right\| \leqq C_{1} t^{-1}, \quad t>0,
$$

which is a necessary and sufficient condition that $A$ is of type $(\omega, M(\theta))$ with $\omega<\pi / 2$ (Yosida [21], Komatsu [12]).

REMARK. If condition (12.4) is satisfied only for small $t$, it follows that for every $\varepsilon>0, A+\varepsilon$ is of type $(\omega, M(\theta))$ with $\omega<\pi / 2$ because the same calculation leads to

$$
\left\|(A+\varepsilon) e^{-\varepsilon t} T_{t}\right\| \leqq C_{2} t^{-1}, \quad t>0 .
$$

Theorem 12.3. Let $A$ and $T_{t}$ be as in Theorem 12.1 and let $\sigma>0$ be not an integer. Then the following conditions for $x$ are equivalent.

(i) For each $\varepsilon>0$, there is a constant $C$ independent of $t$ in the sector $|\arg t| \leqq \pi / 2-\omega-\varepsilon$ such that

$$
\left\|T_{t} A^{n} x-A^{n} x\right\| \leqq C|t|^{\rho} .
$$

(ii) $x \in C^{o}$.

(iii) $x \in D^{o}$.

(iv) For each $\operatorname{Re} \alpha>\sigma$ and $\varepsilon>0$ there is a constant $C$ such that

$$
\left\|A_{+}^{\alpha} T_{t} x\right\| \leqq C|t|^{\sigma-\operatorname{Re} \alpha},
$$

for $|\arg t| \leqq \pi / 2-\omega-\varepsilon$.

(v) (12.6) holds for an $\alpha, \operatorname{Re} \alpha>\sigma$, and sufficiently small $t>0$.

Proof. Implication (i) $\Rightarrow$ (ii) $\Rightarrow$ (iii) and (iv) $\Rightarrow$ (v) are immediate. (iii) $\Rightarrow$ (iv). We may assume that $0<\sigma<1$ without loss of generality. If $x \in D^{\sigma}$, then the estimate

$$
\left\|(\lambda+A)^{-1} x-\lambda^{-1} x\right\| \leqq C|\lambda|^{-\sigma-1}
$$

holds uniformly on each sector $|\arg \lambda| \leqq \pi-\omega-\varepsilon, \varepsilon>0$. In fact, the resolvent equation gives

$$
\lambda\left((\lambda+A)^{-1}-\lambda^{-1}\right) x=\left(1+(\mu-\lambda)(\lambda+A)^{-1}\right) \mu\left((\mu+A)^{-1}-\mu^{-1}\right) x .
$$

If $\lambda$ is in the sector $|\arg \lambda| \leqq \pi-\omega-\varepsilon$, we can choose a $\mu>0$ such that $C_{1}|\lambda| \leqq \mu \leqq C_{2}|\lambda|$ with constants $C_{i}$ independent of $\lambda$. Thus we have

$$
\left\|(\lambda+A)^{-1} x-\lambda^{-1} x\right\| \leqq\left(1+\left(C_{2}+1\right) M(\pi-\omega-\varepsilon)\right) C_{1}^{-\sigma}|\lambda|^{-\sigma-1} q_{\sigma}(x) .
$$

Since $\int_{\Gamma}(-\lambda)^{\alpha} e^{t \lambda} \lambda^{-1} d \lambda=0$, it follows from Theorem 12.1 that 


$$
\begin{aligned}
\left\|A_{+}^{\alpha} T_{t} x\right\| & =\left\|\frac{1}{2 \pi i} \int_{\Gamma}(-\lambda)^{\alpha} e^{t \lambda}\left((\lambda+A)^{-1}-\lambda^{-1}\right) x d \lambda\right\| \\
& \leqq C_{3} \int_{0}^{\infty} r^{\operatorname{Re} \alpha-\sigma-1} e^{-c|t| r} d r \\
& =C_{4}|t|^{\sigma-\operatorname{Re} \alpha} .
\end{aligned}
$$

$(v) \Longrightarrow$ (ii). First we note that if (12.6) holds for an $\alpha$, then it holds for every $\alpha$ with a greater real part. For, if $\operatorname{Re} \beta>0$, then

$$
\left\|A_{+}^{\alpha+\beta} T_{t} x\right\| \leqq\left\|A_{+}^{\beta} T_{t / 2}\right\|\left\|A_{+}^{\alpha} T_{t / 2} x\right\| \leqq C t^{\sigma-\operatorname{Re}(\alpha+\beta)} .
$$

Therefore we have

$$
\left\|(-A)^{m} T_{t} x\right\| \leqq C t^{\sigma-m}, \quad 0<t<t_{0},
$$

for an integer $m>\sigma$. Since $(-A)^{m} T_{t} x$ is the $m$ th derivative of $T_{t} x$, it follows that

$$
\begin{aligned}
& \left\|(-A)^{m-1} T_{t} x-(-A)^{m-1} T_{s} x\right\|=\left\|\int_{s}^{t}(-A)^{m} T_{r} x d r\right\| \\
& \quad \leqq C|\sigma-m+1|^{-1} \cdot\left|t^{\sigma-m+1}-s^{\sigma-m+1}\right|, \quad t, s \in\left(0, t_{0}\right) .
\end{aligned}
$$

If $m-\sigma>1$, we have (12.8) with $m$ replaced by $m-1$. If $m-\sigma<1$, then (12.9) shows that $(-A)^{m-1} T_{t} x$ converges as $t \rightarrow 0$, so that $x$ belongs to $D\left(A^{m-1}\right)$. Letting $s \rightarrow 0$ in (12.9), we obtain also

$$
\left\|T_{t}(-A)^{m-1} x-(-A)^{m-1} x\right\| \leqq C|\sigma-m+1|^{-1} t^{\sigma-m+1} .
$$

This proves $x \in C^{\sigma}$.

The implication (iv) $\Longrightarrow$ (i) is proved similarly.

Even when $\sigma$ is an integer $>0$, the equivalence of (iv) and (v) is proved in the same way.

Definition. When $-A$ generates an analytic semi-group, let us denote by $B^{o}, \sigma>0$, the set of all $x$ such that (v) (or (iv)) of Theorem 12.3 is satisfied.

Then Theorems 7.6 and 11.6 are unified in the following way.

THEOREM 12.4. Let $A$ be as above. If $\sigma>\operatorname{Re} \alpha>0$, then $A_{+}^{\alpha}$ maps $B^{\sigma}$ into $B^{\sigma-\operatorname{Re} \alpha}$. If $\sigma>0$ and $\operatorname{Re} \alpha \leqq 0$, then $A_{0}^{\alpha} \operatorname{maps} B^{\sigma} \cap D\left(A_{0}^{\alpha}\right)$ into $B^{\sigma-\operatorname{Re} \alpha}$. Note that $\sigma-\operatorname{Re} \alpha$ can be an integer.

Proof. Let $x \in B^{\sigma} \cap D\left(A^{\alpha}\right)$. If $\operatorname{Re} \beta>\sigma-\operatorname{Re} \alpha$, we have by Theorems 7.1 or 7.3

$$
\begin{aligned}
\left\|A_{+}^{\beta} T_{t} A_{*}^{\alpha} x\right\| & =\left\|A_{0}^{\beta} A_{*}^{\alpha} T_{t} x\right\| \\
& =\left\|A_{+}^{\alpha+\beta} T_{t} x\right\| \\
& \leqq C|t|^{\sigma-\operatorname{Re}(\alpha+\beta)} .
\end{aligned}
$$


13. Miscellaneous results. In this section we give a few results and applications related to the fractional powers of operators with and without proofs.

Theorem 13.1. Let $X \supset Y$ be two Banach spaces such that the topology of $Y$ is stronger than that of $X$. (By the closed graph theorem it is enough to assume that if $x_{j} \rightarrow x$ in $X$ and $x_{j} \rightarrow y$ in $Y$, then $x=y$.) If a closed linear operator $A$ in $X$ and its maximal restriction $B$ in $Y$ (see Section 1) satisfy assumption (1.5) in $X$ and $Y$ respectively, and if $D(B)$ is dense in $Y$, then $B_{+}^{\alpha}$ is the maximal restriction of $A_{+}^{\alpha}$ in $Y$. If in particular $Y$ is a closed subspace of $X$, then the maximal restriction is the simple restriction to $Y$.

Proof. It follows from the assumptions that $(\lambda+A)^{-1}, \lambda>0$, maps $Y$ into $Y$ and

$$
(\lambda+B)^{-1}=\left.(\lambda+A)^{-1}\right|_{Y}, \quad \lambda>0 .
$$

Hence we have

$$
B_{+}^{\alpha} \lambda^{n}(\lambda+B)^{-n}=\left.A_{+}^{\alpha} \lambda^{n}(\lambda+A)^{-n}\right|_{Y}, \quad n>\operatorname{Re} \alpha .
$$

Let $y \in D\left(B_{+}^{\alpha}\right)$. Then Proposition 4.5 shows

$$
B_{+}^{\alpha} \lambda^{n}(\lambda+B)^{-n} y \rightarrow B_{+}^{\alpha} y \text { in } Y \text { as } \lambda \rightarrow \infty .
$$

so that we have

$$
A_{++}^{\alpha} \lambda^{n}(\lambda+A)^{-n} y \rightarrow B_{+}^{\alpha} y \text { in } X .
$$

Since $y \in \overline{D(B)} \subset \overline{D(A)}$, this proves that $y \in D\left(A_{+}^{\alpha}\right)$ and $A_{+}^{\alpha} y=B_{+}^{\alpha} y$.

Conversely let $y \in D\left(A_{+}^{\alpha}\right) \cap Y$ and $A_{+}^{\alpha} y \in Y$. We have

$$
\begin{aligned}
B_{+}^{\alpha} \lambda^{n}(\lambda+B)^{-n} y & =A_{+}^{\alpha} \lambda^{n}(\lambda+A)^{-n} y \\
& =\lambda^{n}(\lambda+A)^{-n} A_{+}^{\alpha} y \\
& =\lambda^{n}(\lambda+B)^{-n} A_{+}^{\alpha} y .
\end{aligned}
$$

Since $D(B)$ is dense in $Y$, it follows that

$$
B_{+}^{\alpha} \lambda^{n}(\lambda+B)^{-n} y \rightarrow A_{+}^{\alpha} y \text { in } Y \text { as } \lambda \rightarrow \infty .
$$

Therefore we have $y \in D\left(B_{+}^{\alpha}\right)$ and $B_{+}^{\alpha} y=A_{+}^{\alpha} y$.

If $Y$ is a closed subspace, (13.3) holds without the assumption that $A_{+}^{\alpha} y \in Y$. Thus the simple restriction gives the maximal restriction.

The assumptions of Theorem 13.1 are satisfied if $-A$ is the infinitesimal generator of a bounded semi-group $T_{t}$ in $X, Y$ is invariant under $T_{t}$ and the restriction $S_{t}=\left.T_{t}\right|_{Y}$ forms a bounded semi-group in $Y$. In this case $-B$ coincides with the infinitesimal generator of $S_{t}$. 
Let us denote by $c_{0}(\boldsymbol{Z})$ the space of all sequences $x=\left(\xi_{k}\right), k=0$, $\pm 1, \pm 2, \cdots$, such that $\xi_{k} \rightarrow 0$ as $|k| \rightarrow \infty$ and normed by $\|x\|=\sup \left|\xi_{k}\right|$. Then each of $L^{p}(T), 1 \leqq p<\infty$, and $C(T)$ on the unit circle $T$ is embedded in $c_{0}(\boldsymbol{Z})$ by the Fourier transformation. The translation semigroup $S_{t}$ :

$$
S_{t} x\left(e^{i s}\right)=x\left(e^{i(s-t)}\right)
$$

becomes the restriction of the semi-group $T_{t}$ defined by

$$
T_{t}\left(\xi_{k}\right)=\left(e^{-i t k} \xi_{k}\right), \quad\left(\xi_{k}\right) \in c_{0}(\boldsymbol{Z}) .
$$

We denote by $-A$ and $-d / d s$ the infinitesimal generators of $T_{t}$ and $S_{t}$ respectively. By a simple computation it is shown that

$$
\begin{aligned}
& A_{+}^{\alpha}\left(\hat{\xi}_{k}\right)=\left((i k)^{\alpha} \xi_{k}\right), \\
& D\left(A_{+}^{\alpha}\right)=\left\{\left(\xi_{k}\right) \in c_{0}(\boldsymbol{Z}) ;\left((i k)^{\alpha} \xi_{k}\right) \in c_{0}(\boldsymbol{Z}) .\right.
\end{aligned}
$$

Thus we have:

Proposition 13.2. Let $X$ be any one of $L^{p}(T), 1 \leqq p<\infty$, and $C(T)$. Then a function

$$
x\left(e^{i s}\right) \sim \sum_{k=-\infty}^{\infty} c_{k} e^{i k s} \in X
$$

belongs to $D\left((d / d s)_{+}^{\alpha}\right)$ if and only if the formal sum

$$
\left(\frac{d}{d s}\right)^{\alpha} x\left(e^{i s}\right) \sim \sum_{k=-\infty}^{\infty} c_{k}(i k)^{\alpha} e^{i k s}
$$

represents a function in $X$.

The following two theorems may be proved in the same way as Theorem 13.1.

Theorem 13.3. Let $X$ and $Y$ be Banach spacss, and $T$ bo a continuous linear mapping from $X$ to $Y$. If closed linear operators $A$ and $B$ satisfy

$$
B T x=T A x, \quad x \in D(A)
$$

and assumption (1.5) in $X$ and in $Y$, respectively, then we have

$$
B_{*}^{\alpha} T x=T A_{*}^{\alpha} x, \quad x \in D\left(A_{*}^{\alpha}\right),
$$

where $A_{*}^{\alpha}$ has the same subscript as $B_{*}^{\alpha}$.

Theorem 13.4 (Love-Young [14]). Let $X$ be a Banach space and let $X^{\prime}$ be its dual space. If an operator $A$ in $X$ satisfies (1.5) and 
has a dense domain, then its transposed operator $B=A^{\prime}$ also satisfies (1.5) and $B_{+}^{\alpha}$ is the maximal restriction of $\left(A_{+}^{\alpha}\right)^{\prime}$ in $\overline{D(B)}$. If, moreover, $R(A)$ is dense, then $B_{-}^{\alpha}$ and $B_{0}^{\alpha}$ are the maximal restrictions of $\left(A_{0}^{\alpha}\right)^{\prime}$ in $\overline{R(B)}$ and $\overline{D(B)} \cap \overline{R(B)}$, respectively.

Let us apply Theorem 13.3 to the regularity of the convolution of functions. Let $A$ be the negative of the generator of translation semigroup

$$
T_{t} x(s)=x(s-t)
$$

in $B(-\infty, \infty)$, and let $B$ be the corresponding operator in $L^{1}(-\infty, \infty)$.

Proposition 13.5. If $x(s) \in D\left(A_{+}^{\alpha}\right)$ and $y(s) \in D\left(B_{+}^{\beta}\right)$, then the convolution $x * y(s) \in D\left(A_{+}^{\alpha+\beta}\right)$.

Proof. The mapping $T: B(-\infty, \infty) \times L^{1}(-\infty, \infty) \rightarrow B(-\infty, \infty)$ defined by $T(x, y)=x * y$ is a continuous bilinear mapping, and we have

$$
\begin{aligned}
& A(x * y)=A x * y, x \in D(A), y \in L^{1}(-\infty, \infty), \\
& A(x * y)=x * B y, x \in B(-\infty, \infty), y \in D(B) .
\end{aligned}
$$

Therefore it follows from Theorem 13.3 that if $x \in D\left(A_{+}^{\alpha}\right)$ and $y \in L^{1}(-\infty, \infty)$, then $x * y \in D\left(A_{+}^{\alpha}\right)$ and $A_{+}^{\alpha}(x * y)=\left(A_{+}^{\alpha} x\right) * y$. If, moreover, $y \in D\left(B_{+}^{\beta}\right)$, then we have $\left(A_{+}^{\alpha} x\right) * y \in D\left(A_{+}^{\beta}\right)$ and $A_{+}^{\beta}\left(\left(A_{+}^{\alpha} x\right) * y\right)=$ $\left(A_{+}^{\alpha} x\right) *\left(B_{+}^{\beta} y\right)$, so that $x * y$ belongs to $D\left(A_{+}^{\alpha+\beta}\right)$.

Corollary 13.6. Let $x(s) \in B(-\infty, \infty)$ be uniformly Hölder continuous with exponent $0<\alpha<1$ and let $y(s) \in C_{0}(-\infty, \infty)$ be uniformly Hölder continuous with exponent $0<\beta<1$. If $\alpha+\beta>1$, then $x * y(s)$ is differentiable and the derivative is uniformly Hölder continuous with exponent $\alpha+\beta-1-\varepsilon$, for an arbitrary $\varepsilon>0$. If $\alpha+\beta \leqq 1$, then $x * y(s)$ is uniformly Hölder continuous with exponent $\alpha+\beta-\varepsilon, \varepsilon>0$.

THEOREM 13.7. Let

$$
A=\int_{C} \zeta d E(\zeta)
$$

be a normal operator in a Hilbert space $X$. Then $A$ is of type $(\omega, M(\theta))$ if and only if the spectrum $\sigma(A)$ is contained in the sector $|\arg \lambda| \leqq \omega$. If this is the case, $M(\theta)$ satisfies

$$
M(\theta) \leqq \begin{cases}1 & \theta \leqq \pi / 2-\omega \\ \operatorname{cosec}(\omega+\theta), & \theta>\pi / 2-\omega .\end{cases}
$$


In particular, a normal operator is the negative of the generator of $a$ bounded semi-group if and only if $A$ is of type $(\pi / 2, M(\theta))$.

In order that an element $x$ belong to $D^{\sigma}$ it is necessary and sufficient that

$$
q_{\sigma}(x)^{2}=\sup _{0<\lambda<\infty} \lambda^{2 \rho} \int|\lambda+\zeta|^{-2}|\zeta|^{2 n+2} d\|E(\zeta) x\|^{2}<\infty .
$$

When - A generates a semi-group, $D^{o}$ coincides with $C^{\sigma}$.

The fractional power $A^{\alpha}$ is given by

$$
\begin{aligned}
A_{+}^{\alpha} & =\int_{C} \zeta^{\alpha} d E(\zeta), \\
A_{0}^{\alpha} & =\int_{C-\{0\}} \zeta^{\alpha} d E(\zeta) .
\end{aligned}
$$

The domain $D\left(A_{+}^{\alpha}\right)$ consists of the elements $x$ such that

$$
\left\|A_{+}^{\alpha} x\right\|=\int_{C}\left|\zeta^{\alpha}\right|^{2} d\|E(\zeta) x\|^{2}<\infty .
$$

If $\operatorname{Re} \alpha=\operatorname{Re} \beta$, then $\left\|A_{+}^{\alpha} x\right\|$ is equivalent to $\left\|A_{+}^{\beta} x\right\|$ and $D\left(A_{+}^{\alpha}\right)=D\left(A_{+}^{\beta}\right)$. However, $D\left(A_{+}^{\alpha}\right)$ does not coincide with $D^{\mathrm{Re} \alpha}$ if $A$ is unbounded and $\operatorname{Re} \alpha$ is not an integer.

Proof. We prove only the last statement. We have

$$
\lambda^{2 \rho}|\lambda+\zeta|^{-2}|\zeta|^{2} \leqq C|\zeta|^{2 \rho}, \lambda>0,|\arg \zeta| \leqq \omega
$$

with a constant $C$ depending only on $0<\rho<1$ and $\omega$. Thus if $x \in D\left(A_{+}^{\sigma}\right)$ for a $\sigma=n+\rho>0$, we have

$$
q(\lambda, x)=\int \lambda^{2 \rho}|\lambda+\zeta|^{-2}|\zeta|^{2 n+2} d\|E(\zeta) x\|^{2} \leqq C\left\|A_{+}^{\alpha} x\right\|^{2} .
$$

Further it follows from Lebesgue's theorem that

$$
q(\lambda, x) \rightarrow 0 \quad \text { as } \quad \lambda \rightarrow 0 \text {. }
$$

If $A$ is unbounded, however, it is not difficult to construct an element $x$ or a measure $d\|E(\zeta) x\|^{2}$ such that

$$
q(\lambda, x) \leqq C \text { and } q(\lambda, x) \nrightarrow 0 .
$$

This completes the proof.

Let us denote by $H$ the space of functions $x(z)$ which are continuous on the unit disk $|z| \leqq 1$ and analytic in the open disk $|z|<1$, and by $H^{p}, 1 \leqq p<\infty$, the Hardy space $\subset L^{p}(T)$ (Hoffman [6]). Then the translation (or rotation) group $S_{t}$ restricted to $H$ or $H^{p}$ becomes the boundary group of the analytic semi-group 


$$
S_{t} x\left(e^{i s}\right)=x\left(e^{\operatorname{Im} t} e^{i(s-\operatorname{Re} t)}\right), \quad \operatorname{Im} t \leqq 0 .
$$

$-i d / d s$, which is the negative of the infinitesimal generator of $S_{t}$ in the direction of the negative imaginary axis, satisfies the conditions of Section 12 , so that if $\sigma>0$ is not an integer we have

$$
D^{o}\left(\frac{1}{i} \frac{d}{d s}\right)=C^{o}\left(\frac{1}{i} \frac{d}{d s}\right)=B^{o}\left(\frac{1}{i} \frac{d}{d s}\right) .
$$

On the other hand, we have shown in the proof of Theorem 12.3 that $D^{\sigma}(-i d / d s)=D^{\sigma}(d / d s) . \quad C^{\sigma}(-i d / d s)$ coincides with $C^{\sigma}(d / d s)$ because of the identity

$$
S_{2 h}-1=\left(S_{(1-i) h}-1\right)-S_{2 h}\left(S_{(-1-i) h}-1\right)
$$

and the equivalence of (i) and (ii) in Theorem 12.3. Further it follows from the identity

$$
\frac{1}{i} \frac{d}{d s} x\left(r e^{i s}\right)=r e^{i s} \frac{d}{d z} x\left(r e^{i s}\right)
$$

that $\|-i d x / d s\|$ is equivalent to $\|d x / d z\|$ if $r$ is near 1 . Thus we have proved the following theorem.

THeOREM 13.8 (Hardy-Littlewood [5]). Let $X$ be $H$ or $H^{p}$, $1 \leqq p<\infty$, and let $0<\sigma<1$. Then the following conditions for $x \in X$ are equivalent.

(i ) $\left\|\int_{0}^{\infty} \lambda e^{-\lambda t}\left(x\left(e^{i(s-t)}\right)-x\left(e^{i s}\right)\right) d t\right\|=O\left(\lambda^{-\sigma}\right), \lambda \rightarrow \infty$.

(ii) $\left\|x\left(e^{i(s-h)}\right)-x\left(e^{i s}\right)\right\|=O\left(h^{\sigma}\right), h \rightarrow 0$.

(iii) $\left\|x\left(r e^{i s}\right)-x\left(e^{i s}\right)\right\|=O\left((1-r)^{\sigma}\right), r \rightarrow 1$.

(iv) $\left\|\frac{d}{d z} x\left(r e^{i s}\right)\right\|=O\left((1-r)^{\sigma-1}\right), r \rightarrow 1$.

(v) $x \in D^{\sigma}\left(\frac{1}{i} \frac{d}{d s}\right)$.

If $1<p<\infty$, M. Riesz's theorem (Zygmund [24], Hoffman [6]) states that the natural projection $P$ from $L^{p}(T)$ to $H^{p}$ is continuous. Since $P$ is commutative with $(\lambda+d / d s)^{-1}$, we obtain the following theorem.

TheOREM 13.9 (Hardy-Littlewood [5]). If $1<p<\infty$ and $0<\sigma<1$, then the following are equivalent for $x \in L^{p}(T)$.

(i) $x \in D^{o}(d / d s)$.

(ii) $x \in C^{\sigma}(d / d s)$.

(iii) $x=x_{1}+\bar{x}_{2}$, where $x_{i} \in H^{p}$ satisfy one of the conditions of Theorem 13.8 . 
Similarly Privalov's theorem (Zygmund [24]) proves the following theorem.

THEOREM 13.10. A function $x\left(e^{i s}\right) \in C(T)$ is uniformly Hölder continuous with exponent $0<\sigma<1$ if and only if there are two functions $x^{i} \in H$ satisfying the conditions of Theorem 13.8 such that

$$
x\left(e^{i s}\right)=x_{1}\left(e^{i s}\right)+\overline{x_{2}\left(e^{i s}\right)} .
$$

Theorem 9.2 may be applied to the theory of partial differential equations.

Let $A(x, \partial / \partial x)$ be a strongly elliptic linear partial differential operator of order 2 in a domain $\Omega \subset R^{n}$ and let $B(x, \partial / \partial x)$ be a linear partial differential operator on $\partial \Omega$. It is known that if $A(x, \partial / \partial x)$, $B(x, \partial / \partial x)$ and the domain $\Omega$ satisfy certain conditions, then the smallest closed extension $A$ in $L^{p}(\Omega), 1<p<\infty$, of the operator $A(x, \partial / \partial x)$ restricted to the space $\left\{u \in C^{\infty}(\Omega) ; B u=0\right\}$ satisfies:

$$
\begin{gathered}
D(A) \subset W_{L_{p}}^{2}(\Omega), \\
\left\|\nabla^{2} u\right\|_{L_{p}}+\|u\|_{L_{p}} \leqq C_{1}\|A u\|_{L_{p}}, \quad u \in D(A),
\end{gathered}
$$

and $-A$ generates an analytic semi-group.

Let us prove that if $n / p<3$, there are constants $0<\alpha<1$ and $C>0$ such that

$$
\begin{gathered}
\|\nabla u \cdot u\|_{L_{p}} \leqq C\left\|A_{+}^{\alpha} u\right\|_{L_{p}}^{2}, \quad u \in D\left(A_{+}^{\alpha}\right), \\
\|\nabla u \cdot u-\nabla v \cdot v\|_{L_{p}} \leqq C\left(\left\|A_{+}^{\alpha} u\right\|_{L_{p}}+\left\|A_{+}^{\alpha} v\right\|_{L_{p}}\right)\left\|A_{+}^{\alpha}(u-v)\right\|_{L_{p}}, \\
u, v \in D\left(A_{+}^{\alpha}\right) .
\end{gathered}
$$

Obviously we have

$$
\begin{gathered}
\|\nabla u \cdot u\|_{L_{p}} \leqq\|\nabla u\|_{L_{r}}\|u\|_{L_{s}}, \\
\|\nabla u \cdot u-\nabla v \cdot v\|_{L_{p}} \leqq\|\nabla u\|_{L_{r}}\|u-v\|_{L_{s}}+\|\nabla u-\nabla v\|_{L_{r}}\|v\|_{L_{s}},
\end{gathered}
$$

where $p^{-1}=r^{-1}+s^{-1}, r, s \geqq 1$. Let

$$
l=\max \left\{\frac{1}{4}\left(\frac{n}{p}+1\right), \frac{1}{2}\right\}
$$

and choose suitable $r$ and $s$. Then it follows from the SobolevGagliardo-Nirenberg inequality (Nirenberg [15]) that

$$
\begin{aligned}
\|\nabla u\|_{L_{r}} \leqq C\left(\left\|\nabla^{2} u\right\|_{L_{p}}+\|u\|_{L_{p}}\right)^{l}\|u\|_{L_{p}}^{1-l}, \\
\|u\|_{L_{s}} \leqq C\left(\left\|\nabla^{2} u\right\|_{L_{p}}+\|u\|_{L_{p}}\right)^{l}\|u\|_{L_{p}}^{1-l} .
\end{aligned}
$$

Combining these inequalities with (13.9) and applying Theorem 9.2, we 
obtain (13.10) and (13.11).

Once inequalities (13.10) and (13.11) are established, the local existence theorem for the Cauchy problem of the nonlinear equation:

$$
\begin{aligned}
\frac{\partial}{\partial t} u(t, x) & =A\left(x, \frac{\partial}{\partial t}\right) u+\sum_{i=1}^{n} b_{i}(x) \frac{\partial u}{\partial x_{i}} \cdot u, \quad x \in \Omega, \\
B\left(x, \frac{\partial}{\partial x}\right) u(t, x) & =0, \quad x \in \partial \Omega, \\
u(0, x) & =u_{0}(x) \in D\left(A_{+}^{\alpha}\right),
\end{aligned}
$$

is proved by the method of Fujita (Fujita-Kato [3], Itô [8]).

14. Counterexamples. This section is devoted to various counterexamples which show that the statements of some theorems cannot be improved in general.

1. If $X$ is not reflexive,

$$
(\lambda+A)^{-1} x-\lambda^{-1} x=O\left(\lambda^{-2}\right)
$$

does not necessarily imply that $x \in D(A)$. Let $A=-d / d s$ in $B[0, \infty)$. If $x \in B[0, \infty)$ is uniformly Lipschitz continuous, then we have

$$
\begin{aligned}
\left|\left((\lambda+A)^{-1}-\lambda^{-1}\right) x(s)\right| & =\left|\int_{0}^{\infty} e^{-\lambda t}(x(s+t)-x(s)) d t\right| \\
& \leqq C \int_{0}^{\infty} t e^{-\lambda t} d t=C \Gamma(2) \lambda^{-2} .
\end{aligned}
$$

Thus $x$ satisfies (14.1), but it is not always differentiable.

On the other hand, by the same computation, we obtain the following theorem as a corollary of Theorem 2.1.

Theorem 14.1. Let $X$ be a reflexive Banach space and let $-A$ be the generator of a bounded semi-group $T_{t}$ in $X$. Then $x \in D(A)$ if and only if

$$
T_{t} x-x=O(t) \quad \text { as } \quad t \rightarrow 0
$$

In particular a Lipschitz continuous function in $L^{p}(a, b), 1<p<\infty$, is differentiable in $L^{p}(a, b)$ (Hardy-Littlewood [5]).

2. The domain $D(A)$ is not necessarily dense even if $A$ satisfies (1.5). In the space $m$ of all bounded sequences, the operator $A$,

$$
A\left(\xi_{k}\right)=\left(k \xi_{k}\right), D(A)=\{x \in m ; A x \in m\},
$$

gives such an operator. $\overline{D(A)}$ coincides with $c_{0}$. 
3. (3.6) is not true in general. Let $A=-d / d s$ in $B[0, \infty)$. Evidently the null space $N(A)$ is composed of all constant functions. Whereas for any constant $c$,

$$
x(s)=\sin \sqrt{s}+c
$$

does not satisfy

$$
\sup _{s \geq 0} \int_{s}^{\infty} \lambda e^{\lambda(s-t)} x(t) d t \rightarrow 0 \quad \text { as } \quad \lambda \rightarrow 0,
$$

so that $\sin \sqrt{s}$ does not belong to $N(A)+\overline{R(A)}$.

It should be remarked that by a Tauberian theorem (14.3) is equivalent to

$$
\sup _{s \geq 0}\left|\int_{s}^{s+N} x(t) d t\right|=o(N) \quad \text { as } \quad N \rightarrow \infty .
$$

4. The Weierstrass-Hardy function

$$
x\left(e^{i s}\right)=\sum_{k=1}^{\infty} b^{-\rho k} e^{i b^{k_{s}}}, \quad b=2,3, \cdots, 0<\rho<1,
$$

belongs to $C^{p}(d / d s)$ in $H$ and hence in any one of $C(T), H^{p}$ and $L^{p}(T)$, $1 \leqq p<\infty$ (Hardy [4]), but its formal fractional derivative

$$
\left(\frac{d}{d s}\right)_{+}^{\rho} x(s) \sim \sum_{k=1}^{\infty} i^{\rho} e^{i b^{k} s}
$$

is not even in $L^{1}(T)$, so that $x$ does not belong to $D\left((d / d s)_{+}^{\rho}\right)$ in any of the spaces $H, C(T), H^{p}$ and $L^{p}(T)$. Since

$$
\begin{aligned}
\left\|\frac{1}{i} \frac{d}{d s}\left(\lambda+\frac{1}{i} \frac{d}{d s}\right)^{-1} x\right\|_{H} & =\left\|\sum_{k=1}^{\infty} \frac{b^{(1-\rho) k}}{\lambda+b^{k}} e^{i b^{k} s}\right\| \\
& \leqq \sum_{k=1}^{\infty} \frac{b^{(1-\rho) k}}{\lambda+b^{k}} \leqq\left(\frac{1}{1-b^{-\rho}}+\frac{1}{1-b^{\rho-1}}\right) \lambda^{-\rho},
\end{aligned}
$$

the first statement follows from Theorem 13.8.

5. If $\sigma$ is an integer, $B^{\sigma}$ may be wider than $D^{\sigma}$. The function $(1-z)(\log (1-z)-1)$ belongs to $B^{1}(-i d / d s)$ in $H$, but clearly it does not belong to $D^{1}(-i d / d s)$.

6. Contrary to the case of normal operators, $D\left(A_{+}^{\alpha}\right)$ may be different from $D\left(A_{+}^{\beta}\right)$ even if $\operatorname{Re} \alpha=\operatorname{Re} \beta$.

Let $S$ be the shift operator in $c_{0}$ :

$$
S\left(\xi_{1}, \xi_{2}, \xi_{3} \cdots\right)=\left(0, \xi_{1}, \xi_{2}, \cdots\right),
$$


and let

$$
\begin{aligned}
A & =(1-S)^{-1}=1+S+S^{2}+S^{3}+\cdots, \\
A\left(\xi_{1}, \xi_{2}, \xi_{3}, \cdots\right) & =\left(\xi_{1}, \xi_{1}+\xi_{2}, \xi_{1}+\xi_{2}+\xi_{3}, \cdots\right) .
\end{aligned}
$$

The domain $D(A)$ is the set of all sequences $\left(\xi_{k}\right)$ such that $\sum_{k=1}^{\infty} \xi_{k}=0$, which forms a dense subspace. The resolvent is given by

$$
(\lambda+A)^{-1}=\frac{1}{\lambda+1}-\frac{1}{(\lambda+1)^{2}} \sum_{k=1}^{\infty}\left(\frac{\lambda}{\lambda+1}\right)^{k-1} S^{k}, \operatorname{Re} \lambda>0
$$

Since the norm of an operator $P$ of the form

$$
P=\sum_{k=0}^{\infty} c_{k} S^{k}
$$

with a dense domain is given by

$$
\|P\|=\sum_{k=0}^{\infty}\left|c_{k}\right|
$$

the resolvent is estimated by

$$
\begin{aligned}
\left\|(\lambda+A)^{-1}\right\| & =\frac{1}{|\lambda+1|}\left(1+(|\lambda+1|-|\lambda|)^{-1}\right) \\
& \leqq 2\left(\operatorname{Re} \lambda+\frac{1}{2}\right)^{-1}, \quad \operatorname{Re} \lambda>0 .
\end{aligned}
$$

Therefore $A$ is an operator of type $(\pi / 2,2 \sec \theta)$.

By an easy calculation it is proved that

$$
\text { (14.11) } \quad A^{\alpha}=1+\alpha S+\frac{\alpha(\alpha+1)}{2} S^{2}+\frac{\alpha(\alpha+1)(\alpha+2)}{3 !} S^{3}+\cdots
$$

for every complex number $\alpha$.

Since $A^{-1}$ is bounded, $A^{\alpha}$ is bounded for $\operatorname{Re} \alpha<0$, and clearly $A^{0}=1$ is bounded. However, the pure imaginary power $A^{i t}$ is unbounded for any $t \neq 0$, because we have

$$
\begin{aligned}
\left\|A^{i t}\right\| & =1+|i t|+\left|\frac{i t(i t+1)}{2}\right|+\left|\frac{i t(i t+1)(i t+2)}{3 !}\right|+\cdots \\
& \geqq 1+|t|+\frac{1}{2}|t|+\frac{1}{3}|t|+\cdots=\infty .
\end{aligned}
$$

More generally let us prove that

$$
D\left(A^{\sigma+i t}\right) \neq D\left(A^{\sigma}\right), \sigma>0, t>0 .
$$

If $D\left(A^{\sigma+i t}\right) \supset D\left(A^{\sigma}\right)$, there would be a constant $C$ such that 


$$
\left\|A^{\sigma+i t} x\right\| \leqq C\left(\left\|A^{\sigma} x\right\|+\|x\|\right), x \in D\left(A^{\sigma}\right) .
$$

Since $A^{-1}$ is bounded and $D(A)$ is dense, it follows from Corollary 5.3 that $D\left(A^{\sigma}\right)=R\left(A^{-\sigma}\right)$ and that there is a constant $C_{1}$ such that

$$
\left\|A^{o} x\right\|+\|x\| \leqq C_{1}\|y\|, \text { for } x=A^{-\sigma} y \text {. }
$$

Let $y_{j}$ be a sequence in $D\left(A^{i t}\right)$ such that

$$
\left\|y_{j}\right\| \leqq 1,\left\|A^{i t} y_{j}\right\| \geqq j, j=1,2, \cdots .
$$

Theorem 7.3 proves that $A^{-\sigma} y_{j} \in D\left(A^{\sigma+i t}\right)$ and $A^{\sigma+i t} A^{-\sigma} y_{j}=A^{i t} y_{j}$. Thus we have by (14.13) and (14.14)

$$
\left\|A^{i t} y_{j}\right\|=\left\|A^{\sigma+i t} A^{-\sigma} y_{j}\right\| \leqq C C_{1}\left\|y_{j}\right\|,
$$

which contradicts (14.15).

7. In the statement of Theorem 9.2 " $\operatorname{Re} \alpha>\theta \operatorname{Re} \beta$ " cannot be replaced by "Re $\alpha \geqq \theta \operatorname{Re} \beta$ ". In view of Theorem 8.1 we have

$$
\left\|A^{\sigma+i t} x\right\| \leqq C\|A x\|^{\sigma}\|x\|^{1-\sigma}, x \in D(A), 0<\sigma<1 .
$$

But (14.13) does not necessarily hold.

8. Although the operator $A$ in 6 is of type $(\pi / 2,2 \sec \theta),-A$ is not an infinitesimal generator of any semi-group of class $(A)$.

If $-A$ generates a semi-group $\exp (-t A)$, then we have

$$
\exp (-t A) x=\frac{1}{2 \pi i}(C, 1)-\int_{-i \infty}^{i \infty} e^{\lambda t}(\lambda+A)^{-1} x d \lambda, x \in D\left(A^{2}\right) .
$$

(See Hille-Phillips [7].) Since $D\left(A^{2}\right)$ is dense, and $(\lambda+A)^{-1}$ has expansion (14.8), it follows that $\exp (-t A)$ must have the form

$$
\exp (-t A)=\exp (-t)+\sum_{k=1}^{\infty} f_{k}(t) S^{k}
$$

where

$$
\begin{aligned}
f_{k}(t) & =\frac{-1}{2 \pi i} \int_{-i \infty}^{i \infty} e^{\lambda t} \lambda^{k-1}(\lambda+1)^{-k-1} d \lambda \\
& =\sum_{h=0}^{k-1}(-1)^{h+1}\left(\begin{array}{c}
k-1 \\
h
\end{array}\right) \frac{t^{h+1}}{(h+1) !} \exp (-t) .
\end{aligned}
$$

If $\exp (-t A)$ is bounded, $\sum_{k=1}^{\infty}\left|f_{k}(t)\right|$ is finite, so that the function $\psi(z)=\sum_{k=1}^{\infty} f_{k}(t) z^{k}$ must be continuous on the disk $|z| \leqq 1$. We have, however, 


$$
\begin{aligned}
\psi(z) & =\sum_{h=0}^{\infty}(-1)^{h+1} \frac{t^{h+1}}{(h+1) !} \exp (-t) \sum_{k=h+1}^{\infty}\left(\begin{array}{c}
k-1 \\
h
\end{array}\right) z^{k} \\
& =\sum_{h=0}^{\infty}\left(\frac{-z}{1-z}\right)^{h+1} \frac{t^{h+1}}{(h+1) !} \exp (-t) \\
& =\left(\exp \left(\frac{-z t}{1-z}\right)-1\right) \exp (-t)
\end{aligned}
$$

This shows that $\psi(z)$ is discontinuous at $z=1$.

An example of operators of this kind has been given by Phillips (Hille-Phillips [6]), but this example is more interesting because $-A^{-1}=-1+S$ generates a continuous contraction semi-group

$$
\begin{aligned}
\exp \left(-t A^{-1}\right) & =\sum_{k=0}^{\infty} \frac{(-t)^{k}}{k !} \exp (-t) S^{k}, \\
\left\|\exp \left(-t A^{-1}\right)\right\| & =\exp (|t|-\operatorname{Re} t) .
\end{aligned}
$$

9. Even if $-A$ generates a contraction semi-group,

$$
\left\|A_{+}^{\gamma} x\right\| \leqq\|A x\|^{\gamma}\|x\|^{1-\gamma}, 0<\gamma<1, x \in D(A)
$$

does not necessarily hold. Let $A=1-S, \gamma=1 / 2$ and $x=(1,2,1,0,0, \cdots)$. Then we have

$$
\begin{aligned}
A x & =(1,1,-1,-1,0,0, \cdots), \\
A^{1 / 2} x & =(1,3 / 2,-1 / 8, \cdots) .
\end{aligned}
$$

Thus

$$
\left\|A^{1 / 2} x\right\|=3 / 2>\|A x\|^{1 / 2}\|x\|^{1 / 2}=\sqrt{2} .
$$

10. If $X$ is not sequentially weakly complete, Theorem 11.5 may be false without assumption (11.11). The semi-group

$$
T_{t}\left(\xi_{k}\right)=\left(e^{-k t} \xi_{k}\right),\left(\xi_{k}\right) \in c_{0}
$$

has the infinitesimal generator

$$
-A\left(\xi_{k}\right)=\left(-k \xi_{k}\right), D(A)=\left\{\left(\xi_{k}\right) \in c_{0} ; A\left(\xi_{k}\right) \in c_{0}\right\} .
$$

The element $x=(1,1 / 2,1 / 3, \cdots)$ does not belong to $D(A)$, but

$$
\left\langle T_{s} x, y^{\prime}\right\rangle=\sum_{k=1}^{\infty} e^{-k s} \eta_{k} / k
$$

is differentiable for every $y^{\prime}=\left(\eta_{k}\right) \in l^{1}$ and

$$
-\frac{d}{d s}\left\langle T_{s} x, y^{\prime}\right\rangle=\sum_{k=1}^{\infty} e^{-k s} \eta_{k} \in B[0, \infty) \text {. }
$$


REMARKS ADDED ON REVISION. Suppose $\sigma>0$ is not an integer. Let us denote by $D_{*}^{\sigma}\left(C_{*}^{\sigma}\right)$ the set of all $x \in D^{\sigma}\left(C^{\sigma}\right)$ which satisfies stricter estimate

$$
\begin{aligned}
R_{n}(\lambda, x) & =o\left(\lambda^{-\sigma-1}\right), \lambda \rightarrow \infty \\
\left(T_{t} A^{n} x-A^{n} x\right. & \left.=o\left(t^{\rho}\right), t \rightarrow 0\right) .
\end{aligned}
$$

Then we have

$$
D_{*}^{\sigma} \supset D\left(A_{+}^{\alpha}\right), \operatorname{Re} \alpha=\sigma
$$

and, if $-A$ generates a bounded semi-group,

$$
D_{*}^{\sigma} \supset C_{*}^{\sigma} \supset D\left(A_{+}^{\alpha}\right), \operatorname{Re} \alpha=\sigma .
$$

The inclusion $D_{*}^{\sigma} \supset C_{*}^{\sigma}$ is proved in the same way as in the proof of Proposition 11.2. Since $D_{*}^{\sigma}\left(C_{*}^{\sigma}\right)$ is a closed subspace of $D^{\sigma}\left(C^{\sigma}\right)$ with the norm $\|x\|+q_{\sigma}(x)\left(\|x\|+p_{o}(x)\right)$ and contains every $D^{\tau}\left(C^{\tau}\right)$ with $\tau>\sigma$, the other inclusions follow from the estimates

$$
\begin{aligned}
& \|x\|+q_{\sigma}(x) \leqq C\left(\|x\|+\left\|A_{+}^{\alpha} x\right\|\right), \\
& \|x\|+p_{\sigma}(x) \leqq C\left(\|x\|+\left\|A_{+}^{\alpha} x\right\|\right)
\end{aligned}
$$

and the definition of $A_{+}^{\alpha}$.

$$
D_{*}^{\mathrm{Re} \alpha}=C_{*}^{\mathrm{Re} \alpha}=D\left(A_{+}^{\alpha}\right)
$$

holds for the operator $A$ of Example 10 of Section 14 .

In the space $D_{*}^{\sigma}$ the approximation theorem (Lemma 2.4) holds in the norm topology. Thus if we used $D_{*}^{\sigma}$ instead of $D^{\sigma}$ we could avoid the strange topology of $D^{\sigma}$.

\section{REFERENCES}

1. A. V. Balakrishnan, An operational calculus for infinitesimal generators of semigroups, Trans. Amer. Math. Soc. 91 (1959), 330-353.

2. - Fractional powers of closed operators and the semi-groups generated by them, Pacific J. Math. 10 (1960), 419-437.

3. H. Fujita and T. Kato, On the Navier-Stokes initial value problem, I, Arch. Rat. Mech. Anal. 16 (1964), 269-315.

4. G. H. Hardy, Weierstrass's non-differentiable function, Trans. Amer. Math. Soc. 17 (1916), 301-325.

5. G. H. Hardy and J. E. Littlewood, Some properties of fractional integrals, I, Math. Z., 27 (1928), 565-606, II, ibd. 34 (1932), 403-439.

6. E. Hille and R. S. Phillips, Functional analysis and semi-groups, Amer. Math. Soc. Colloq. Publ. 31.

7. K. Hoffman, Banach spaces of analytic functions, Prentice Hall, 1962.

8. S. Itô, The existence and the uniqueness of regular solution of non-stationary NavierStokes equation, J. Fac. Sci. Univ. Tokyo, Sec. I 9 (1961), 103-140.

9. T. Kato, Remarks on pseudo-resolvents and in finitesimal generators of semi-groups, 
Proc. Japan Acad. 35 (1959), 467-468.

10. - Note on fractional powers of linear operators, Proc. Japan Acad. 36 (1960), 94-96.

11. - Fractional powers of dissipative operators, J. Math. Soc. Japan 13 (1961), 246-274.

12. H. Komatsu, Semi-groups of operators in locally convex spaces, J. Math. Soc. Japan 16 (1964), 230-262.

13. M. A. Krasnosel'skii and P. E. Sobolevskii, Fractional powers of operators acting in Banach spaces, Doklady Akad. Nauk SSSR 129 (1959), 499-502.

14. E. R. Love and L. C. Young, On fractional integration by parts, Proc. London Math. Soc. 44 (1938), 1-35.

15. L. Nirenberg, On elliptic partial differential equation, Ann. Scu. Norm. Sup. Pisa, Ser. III 13 (1959), 115-162.

16. R. S. Phillips, On the generation of semi-groups of linear operators, Pacific J. Math. 2 (1952), 343-369.

17. M. Sato, Theory of hyperfunctions, I, J, Fac. Sci. Univ. Tokyo, Sec. 18 (1959), 139-193, II, ibd. 8 (1960), 387-437.

18. A. E. Taylor, Spectral theory of closed distributive operators, Acta Math. 84 (1950), 189-224.

19. H. F. Trotter, Approximation of semi-groups of operators, Pacific J. Math. 8 (1958), 887-919.

20. J. Watanabe, On some properties of fractional powers of linear operators, Proc. Japan Acad. 37 (1961), 273-275.

21. K. Yosida, On the differentiability of semi-groups of linear operators, Proc. Japan Acad. 34 (1958), 337-340.

22. - Fractional powers of infinitesimal generators and the analyticity of the semi-groups generated by them, Proc. Japan Acad. 36 (1960), 86-89.

23. — - Ergodic theorem for pseudo-resolvents, Proc. Japan Acad. 37 (1961), 422425.

24. A. Zygmund, Trigonometrical series, Warszawa, 1935.

Received March 17, 1965 and in revised form July 21, 1965. This research was supported in part by the United States National Science Foundation Grant GP-1883.

STANFORD UNIVERSITY AND

UNIVERSITY OF TOKYO 


\title{
PACIFIC JOURNAL OF MATHEMATICS
}

\author{
EDITORS
}

\section{H. SAMELSON}

Stanford University

Stanford, California

J. P. JANS

University of Washington

Seattle, Washington 98105
J. DugundJI

University of Southern California

Los Angeles, California 90007

RICHARD ARENS

University of California

Los Angeles, California 90024

\section{ASSOCIATE EDITORS}

\section{E. F. BECKENBACH}

B. H. NEUMANN

F. WOLF

K. YosidA

\section{SUPPORTING INSTITUTIONS}

\author{
UNIVERSITY OF BRITISH COLUMBIA \\ CALIFORNIA INSTITUTE OF TECHNOLOGY \\ UNIVERSITY OF CALIFORNIA \\ MONTANA STATE UNIVERSITY \\ UNIVERSITY OF NEVADA \\ NEW MEXICO STATE UNIVERSITY \\ OREGON STATE UNIVERSITY \\ UNIVERSITY OF OREGON \\ OSAKA UNIVERSITY \\ UNIVERSITY OF SOUTHERN CALIFORNIA
}

\author{
STANFORD UNIVERSITY \\ UNIVERSITY OF TOKYO \\ UNIVERSITY OF UTAH \\ WASHINGTON STATE UNIVERSITY \\ UNIVERSITY OF WASHINGTON \\ AMERICAN MATHEMATICAL SOCIETY \\ CHEVRON RESEARCH CORPORATION \\ TRW SYSTEMS \\ NAVAL ORDNANCE TEST STATION
}

Mathematical papers intended for publication in the Pacific Journal of Mathematics should be typewritten (double spaced). The first paragraph or two must be capable of being used separately as a synopsis of the entire paper. It should not contain references to the bibliography. Manuscripts may be sent to any one of the four editors. All other communications to the editors should be addressed to the managing editor, Richard Arens at the University of California, Los Angeles, California 90024.

50 reprints per author of each article are furnished free of charge; additional copies may be obtained at cost in multiples of 50 .

The Pacific Journal of Mathematics is published monthly. Effective with Volume 16 the price per volume (3 numbers) is $\$ 8.00$; single issues, $\$ 3.00$. Special price for current issues to individual faculty members of supporting institutions and to individual members of the American Mathematical Society: $\$ 4.00$ per volume; single issues $\$ 1.50$. Back numbers are available.

Subscriptions, orders for back numbers, and changes of address should be sent to Pacific Journal of Mathematics, 103 Highland Boulevard, Berkeley 8, California.

Printed at Kokusai Bunken Insatsusha (International Academic Printing Co., Ltd.), No. 6, 2-chome, Fujimi-cho, Chiyoda-ku, Tokyo, Japan.

PUBLISHED BY PACIFIC JOURNAL OF MATHEMATICS, A NON-PROFIT CORPORATION

The Supporting Institutions listed above contribute to the cost of publication of this Journal, but they are not owners or publishers and have no responsibility for its content or policies. 


\section{Pacific Journal of Mathematics \\ Vol. 19, No. $2 \quad$ June, 1966}

Leonard Daniel Baumert, Extreme copositive quadratic forms . ......... 197

Fred James Bellar, Jr., Pointwise bounds for the second initial-boundary value problem of parabolic type ........................ 205

L. Carlitz and David Paul Roselle, Restricted bipartite partitions ........ 221

Robin Ward Chaney, On the transformation of integrals in measure space ........................................... 229

Colin W. Clark, An embedding theorem for function spaces ........... 243

Edwin Duda, A theorem on one-to-one mappings................ 253

Ben Fitzpatrick, Jr. and Donald Reginald Traylor, Two theorems on metrizability of Moore spaces.......................... 259

Allen Roy Freedman, An inequality for the density of the sum of sets of vectors in $n$-dimensional space ....................... 265

Michael Friedberg, On representations of certain semigroups .......... 269

Robert William Gilmer, Jr., The pseudo-radical of a commutative ring . . . . 275

Hikosaburo Komatsu, Fractional powers of operators ............... 285

Daniel Rider, Transformations of Fourier coefficients ................. 347

David Alan Sánchez, Some existence theorems in the calculus of variations ...................................... 357

Howard Joseph Wilcox, Pseudocompact groups............... 365

William P. Ziemer, Some lower bounds for Lebesgue area ............. 381 